\title{
DETECTION OF STARS WITHIN 0.8 in OF Kepler OBJECTS OF INTEREST
}

\author{
Rea Kolbl ${ }^{1}$, Geoffrey W. Marcy ${ }^{1}$, Howard IsaAcson ${ }^{1}$, and Andrew W. Howard ${ }^{2}$ \\ ${ }^{1}$ University of California, Berkeley, CA 94720, USA \\ 2 Institute for Astronomy, University of Hawaii, Honolulu, HI 96822, USA \\ Received 2014 May 7; accepted 2014 September 19; published 2014 December 15
}

\begin{abstract}
We present an algorithm to search for the faint spectrum of a second star mixed with the spectrum of a brighter star in high resolution spectra. We model optical stellar spectra as the sum of two input spectra drawn from a vast library of stars throughout the H-R diagram. From typical spectra having a resolution of $\mathrm{R}=60,000$, we are able to detect companions as faint as $1 \%$ relative to the primary star in approximately the $\mathrm{V}$ and $\mathrm{R}$ bandpasses of photometry. We are also able to find evidence for triple and quadruple systems, given that any additional companions are sufficiently bright. The precise threshold percentage depends on the signal-to-noise of the spectrum and the properties of the two stars. For cases of non-detection, we place a limit on the brightness of any potential companions. This algorithm is useful for detecting faint orbiting companions and background stars that are angularly close to a foreground target star. The size of the entrance slit to the spectrometer, $0.87 \times 3$ arcsec (typically), sets the angular domain within which the second star can be detected. We analyzed Keck-HIRES spectra of 1160 California Kepler Survey objects of interest (KOI) searching for the secondary spectra, with the two goals of alerting the community to two possible host stars of the transiting planet and to dilution of the light curve. We report 63 California KOI showing spectroscopic evidence of a secondary star.
\end{abstract}

Key words: binaries: spectroscopic - methods: statistical - planetary systems - stars: individual (SB2) techniques: radial velocities - techniques: spectroscopic

\section{INTRODUCTION}

One of the Kepler mission's main goals is to determine the abundance of the terrestrial and larger planets in the habitable zone, characterize their orbits, and determine their physical properties. As of 2012 February 27, the catalog of Kepler Planetary Candidates has 2300 entries (Batalha et al. 2013). Planet candidates are identified by their photometric signals and then confirmed by additional photometric and spectroscopic analysis. However, it is well known that roughly half of all star systems are actually binaries or triples (Raghavan et al. 2010). Therefore, it is likely that roughly half of the Kepler Planetary Candidates have two or more possible host stars. At the typical distances of Kepler objects of interest (KOIs) of $0.3-1.0 \mathrm{kpc}$, orbiting stellar companions with typical orbital distances of 5-500 AU would be unresolved from the primary stars. This implies that roughly half of the Kepler Planetary Candidates have a major ambiguity about the nature of the host star of the transiting: it could be any of the stars in the binary or triple star system. Moreover, even if the planet transits the primary star, the dilution of the Kepler photometry from the secondary and tertiary stars causes an underestimation of the radius of the planet. Such an ambiguity can also be caused by a background star that is angularly close to the Kepler target. The identification of a second set of absorption lines in the spectrum of a KOI highlights cases with such ambiguities, and casts appropriate uncertainty on the measured radii of the planets.

Besides the dilution of the Kepler light curve, there is also a possibility that what appears to be a planet is in fact a false positive. This is a serious concern for the Kepler mission, because there are numerous astrophysical phenomena that can produce the dimming that are indistinguishable from that of the transiting planet. A few such examples include the grazing eclipsing binaries, a giant primary star eclipsed by a dwarf, or a background star. The first two cases can usually be identified by photometry alone, but the background stars pose further challenges to planet validations, particularly for cases of background eclipsing binaries. There is also another case that is especially important to consider for smaller planet candidates: The amount of dimming caused by an Earth-like planet orbiting a Sun-like star can be mimicked by a larger planet orbiting a background star, or a less bright binary system companion.

These alternative situations can drastically alter the interpretation of the phenomena that caused the dimming detected by the Kepler spacecraft, and significant efforts have been made to characterize the false positive probability (FPP) among the Kepler planet candidates. The values in the literature range from FPP $<10 \%$ (Morton \& Johnson 2011; Fressin et al. 2013) to $\sim 35 \%$ (Santerne 2012) for giant planets. Even the lowest estimated FPP is high enough to raise concerns about individual planet discoveries. In an effort to decrease FPP, we have developed a method for detecting faint stellar companions in double-lined spectroscopic binaries.

In this paper, we present a spectroscopic method of searching for a secondary set of absorption lines in a spectrum. A detection of such lines would reveal a secondary star that is close enough for its light to reach the aperture and affect the interpretation of the phenomena responsible for the dimming. The entrance slit has angular dimensions projected onto the sky of $0.87 \mathrm{in} \times 3.0 \mathrm{in}$. The slit is oriented differently for each exposure, with the primary star centered on the slit in both dimensions. A secondary star will be included in the spectrum if it resides anywhere within the slit, which restricts the maximum angular separation between the primary star and the secondary star at 0.43 in to $1.5 \mathrm{in}$, depending on the orientation of the slit. Most commonly, the secondary star is 
oriented relative to the primary star at a typical angle of $30-60^{\circ}$ to the length of the slit, in which case its light will be collected if it resides within 0.5 in-0.8 in from the primary star.

Our goal is to detect any bound or non-bound secondary star as faint as $1 \%$ relative to the primary target star, with either sufficiently large relative radial velocity to allow for the Doppler separation of the two sets of absorption lines, or with sufficiently different spectral features to allow for the differentiation among the overlapping spectral lines. The presence of such a star does not prove that the transit signal is not a planet. It merely indicates that a second star is located within 0.5 in -0.8 in of the primary star, causing ambiguity about the host star and uncertainty about the planet radius.

A standard tool for spectroscopic binaries is TODCOR (Mazeh et al. 1993), which is used extensively to model, selfconsistently, the two underlying spectra in a given spectrum. This code has been shown to provide excellent detection and assessment of the properties of the two stars. However, we wanted to develop our own method for detecting secondary lines, with a primary goal of being extremely conservative in our detections. We adopted an "Occam's Razor" approach in which any observed spectrum of just one star would be deemed a sufficient model. We only invoke the spectrum of a second star in the model if absolutely forced to do so by residuals that show the second star to be statistically significant. Thus, we seek an algorithm that accomplishes a statistically robust identification of secondary lines. We were dedicated to constructing an algorithm that we understood in precise detail, and consider our intimate knowledge of the algorithm to be vital in assessing its integrity and functionality.

\section{SPECTRUM PREPARATION}

We worked with spectra from HIRES at Keck Observatory. HIRES has a bandwidth that covers wavelengths from 3643 to $7990 \AA$. We worked with the wavelength region from 4977 to $7990 \AA$, avoiding the parts of the spectrum polluted by telluric lines, as well as the region of the interstellar sodium D lines. This wavelength domain approximately encompasses the $\mathrm{V}$ and $\mathrm{R}$ broadbands of classical photometry.

Using one of the 0.86 in wide slits, HIRES has a resolving power of 60,000 at $5500 \AA$. The signal-to-noise $(\mathrm{S} / \mathrm{N})$ of the spectra depends on the brightness of the primary star and the duration of the exposure. Typical $\mathrm{S} / \mathrm{N}$ values range from $\sim 45$ to 200 per pixel.

First, we continuum-normalize each HIRES spectrum to remove the somewhat variable blaze function from each spectral order. We fit the continuum using the fifth order polynomial, divide the spectrum by the fits and thus obtain a flat continuum at unity, as shown in Figure 1.

Once flattened, we resample the spectrum on a constant $\Delta \lambda / \lambda$ wavelength scale using interpolation. We use a wavelength scale with a pixel spacing of $\sim 1.3 \mathrm{~km} \mathrm{~s}^{-1}$. Resampling is done with a set prescription of converting HIRES pixels into $\Delta \lambda / \lambda$ intervals. This wavelength scale allows for a simpler relationship between the pixel positions of absorption lines and the Doppler shift of the star; with spectrum on a constant $\Delta \lambda / \lambda$ scale, a Doppler shift of $v \mathrm{~km} \mathrm{~s}^{-1}$ corresponds to a simple translation of the spectrum for $\mathrm{N}_{\text {pixels }}=\left(v \mathrm{~km} \mathrm{~s}^{-1}\right) / 1.3$.

\section{THE SEARCH ALGORITHM FOR A FAINT SECONDARY SPECTRUM}

\subsection{Fitting the Spectrum of the Primary Star}

Since we are interested in the relative radial velocity between the primary star and the potential companion, we correct for the Doppler shift of the primary star such that the primary star's absorption lines reside at the rest frame wavelengths. We determine the Doppler shift of the primary star using the $\chi^{2}$ statistic of cross-correlating the corrected NSO Solar spectrum (Wallace et al. 2011) with no Doppler shift. We determine the location of the $\chi^{2}$ minimum, which corresponds to the radial velocity at which the absorption lines of the Sun's spectrum align with the absorption lines of the primary star-the primary star's Doppler shift. Since we are using a wide range of wavelengths, the discrepancy in the spectral type between the Sun and the star in question does not cause any difficulties in determining the radial velocity of the primary star.

\subsubsection{Finding the Best-fit}

Once the spectrum is normalized and on a constant $\Delta \lambda / \lambda$ wavelength scale, we search through the SpecMatch library of stellar spectra to find the best fit for the primary star. The SpecMatch library consists of Keck-HIRES spectra of 640 FGKM stars scattered throughout the H-R diagram, with a concentration to the main sequence and subgiants. These spectra were obtained as part of the California Planet Search. Each spectrum has $\mathrm{S} / \mathrm{N}=100-200$ per pixel between 3800 and $8000 \AA$ with the same spectral resolution as the bulk of the spectra analyzed here, $\mathrm{R}=60,000$.

All of the SpecMatch library spectra are available online at the Keck Observatory Archive. Apart from M dwarfs, each spectrum was analyzed with an advanced version of the spectroscopic analysis package, SME, as described in Valenti $\&$ Fischer (2005). SME was later modified based on the available parallax information, for a revised, improved version of SME (Brewer et al. 2014, in preparation). This analysis yielded the values of $T_{\text {eff }}, \log g$, and [Fe/G] for all FGK stars in the library. Stellar parameters for $\mathrm{M}$ dwarfs were adopted from Rojas-Ayala et al. (2012).

SpecMatch stellar parameter values range in $T_{\mathrm{eff}}=$ $[3250,7260], \log g=[1.46,5.00]$, and $[\mathrm{Fe} / \mathrm{H}]=[-1.475,0.558]$. We assume that the primary star lies on main sequence and exclude the subgiants from the library as possible best-fit candidates. This choice was made due to the preferential choice of subgiants as the best-fitting stars, even when the observed primary star was on main sequence. Excluding the giants improves the quality of the best fit and allows for a better subtraction of the primary spectrum, which is explained in Section 3.3.1. This decision was made with our efforts in mind; since our primary goal is to search for the secondary lines in the spectrum, we sacrifice some of the accuracy of the primary star parameters in order to achieve a better fit to the primary star absorption lines. Nevertheless, if the spectral type of the primary star is known, the algorithm can also be modified to include the subgiants in the fitting process. 

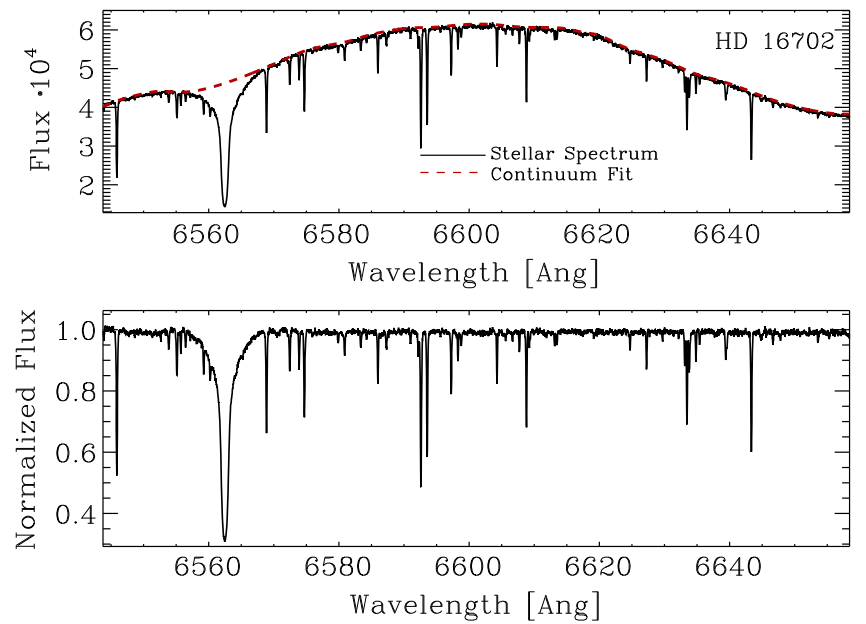

Figure 1. Continuum flattening example using the $H_{\alpha}$ region of the Kepler-16 spectrum. Above: Original spectrum with the over-plotted fifth order polynomial continuum fit, shown as a red dashed line. Below: The normalized and flattened spectrum, obtained by dividing the original spectrum shown above by the continuum fit.

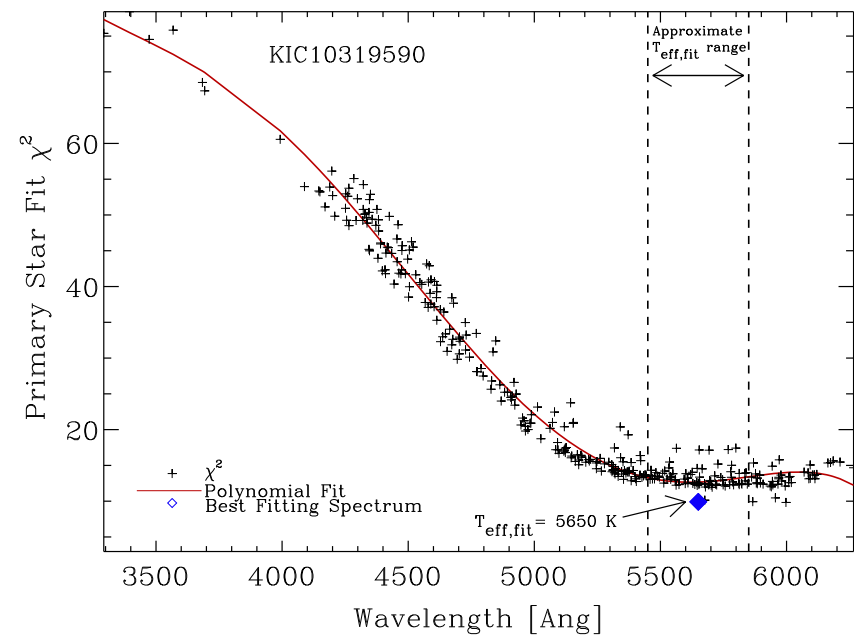

Figure 2. Primary star fit $\chi^{2}$ as a function of a library star's effective temperature. Value of $\chi^{2}$ for each library star is shown as a black " + " sign. Due to the unknown uncertainties of each point along the spectra, we only look at the $\chi^{2}$ values of one spectrum relative to another. The approximate $T_{\text {eff }}$ range of the primary star is determined based on the minimum of the polynomial fit to the $\chi^{2}$ vs. temperature trend. The fit is shown as a red solid line. Within \pm 400 $\mathrm{K}$ from the minimum of the fit, marked with vertical dashed lines, we then choose the best-fitting library spectrum as the spectrum that produced the lowest $\chi^{2}$ value. $T_{\text {eff }}$ of the best fitting spectrum for KIC 10319590 was $5650 \mathrm{~K}$, shown as the blue diamond.

We look for the library spectrum that produces the lowest $\chi^{2}$ value when the absorption lines of the library spectrum are aligned with the studied spectrum. We vary the rotational broadening of each library spectrum and the depth of the absorption lines by diluting the spectrum. The latter is to correct for the finite grid spacing of the metallicity in our library, and is implemented to ensure as complete subtraction of the primary star absorption lines as possible. Due to the inclusion of the constant dilution factor, we are unable to determine the $\log g$ of the primary star. However, as mentioned, the determination of the parameters for the primary star is beyond the scope of this paper, but it can be performed using a different method if so desired.

Taking the above into account, the $\chi^{2}$ is computed using

$$
\chi^{2}=\sum_{i}\left(S_{i}^{\mathrm{lib}}-d \cdot\left(S_{i+p}^{\mathrm{obs}}(v \sin i)-1\right)+1\right)^{2},
$$

where $S^{\text {lib }}$ is the SpecMatch library spectrum, $S^{\text {obs }}(v \sin i)$ is the rotationally broadened observed spectrum, $d$ is the dilution factor, and $p$ is the Doppler shift of the primary star in pixels.

Discrepancies between each library star and the actual spectrum are both due to Poisson noise and intrinsic spectral differences. This is mostly due to the finite grid spacing in the parameter space of our library. Consequently, we cannot establish a good uncertainty estimate for each point along the spectrum, and the actual value of $\chi^{2}$ bears no significance; rather, it is the relative $\chi^{2}$ among all the library spectra that we are concerned with.

In order to prevent outliers from being identified as bestfitting stars, we first identify the approximate temperature of the primary star by finding the minimum of the $\chi^{2}$ versus library star temperature distribution, as shown in Figure 2. The vertical scatter of the points at the same $T_{\text {eff }}$ is due to discrepancies in other parameters, such as $\log g$ and metallicity. Note that we could also plot $\chi^{2}$ as a function of other parameters as well; we choose the temperature as the distribution that has the most easily identifiable minimum.

Based on the polynomial fit to the $\chi^{2}$ distribution we eliminate the outliers that differ from the polynomial fit for more than $3 \sigma$ at any value of the effective temperature. We then repeat the polynomial fitting, and choose the lowest $\chi^{2}$ value within the $\pm 200 \mathrm{~K}$ range about the minimum of the polynomial fit as our best-fitting library spectrum. While restricting the range of possible $T_{\text {eff }}$ to $\pm 200 \mathrm{~K}$ around the polynomial minimum could introduce larger uncertainty in the determined $T_{\text {eff }}$ of the primary star, it does ensure that the chosen library spectrum is the best achievable fit to the observed spectrum and has approximately correct effective temperature. The restricted range excludes any outliers that have not yet been eliminated at the edges of the effective temperature range $(>6100 \mathrm{~K}$ and $<3400)$ due to the edge effects on the shape of the polynomial.

Because the main goal of the primary star fitting is the subtraction of its absorption lines from the spectrum, we do not report any of the best-fit parameters for the primary star. The accuracy of these results is compromised due to several manipulations of the library spectra to account for the finite grid of our library parameter space. Nevertheless, all the described steps do ensure that the primary star absorption lines are fitted almost perfectly and subtracted almost completely. If the parameters for the primary star are known, however, any or all of the values in the parameter space we search over $(\log g$, $[\mathrm{Fe} / \mathrm{H}]$, rotational broadening, $\left.T_{\text {eff }}\right)$ can be constrained to a known range or fixed to a known value.

\subsection{Bright Secondary Stars}

Brighter stellar companions ( $>\sim 10 \%$ relative brightness) can be detected using primary $\chi^{2}$ as a function of Doppler shift. We use the same $\chi^{2}$ function we used for determining 

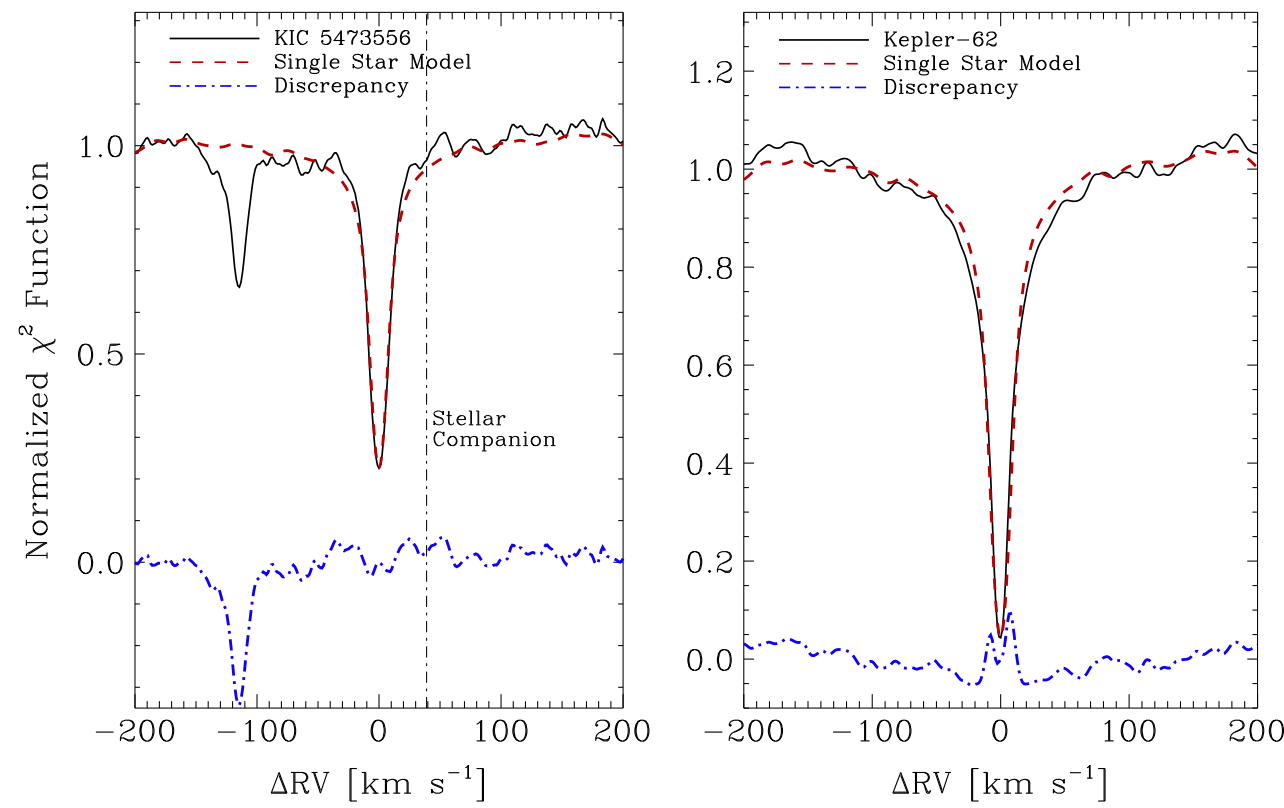

Figure 3. $\chi^{2}$ as a function of Doppler shift for the fit to the spectrum for two stars, KIC 5473556 (left) and Kepler-62 (right). We compute the $\chi^{2}$ between the Sun's and a star's spectrum for a range of Doppler shifts. The dip at $\Delta \mathrm{RV}=0 \mathrm{~km} \mathrm{~s}^{-1}$ corresponds to the alignment of primary star absorption lines with those of the Sun. The red dashed line corresponds to the $\chi^{2}$ function of a Sun spectrum with itself, thus representing a characteristic shape for a single star $\chi^{2}$ function. The blue dotteddashed line shows the difference between the actual $\chi^{2}$ and the characteristic function. Left: KIC 5473556 is a binary system, and we can see the secondary dip at $\Delta \mathrm{RV} \approx 99 \mathrm{~km} \mathrm{~s}^{-1}$. Right: Kepler-62 is a single star, thus its $\chi^{2}$ function matches closely the shape of a characteristic single star $\chi^{2}$ function.

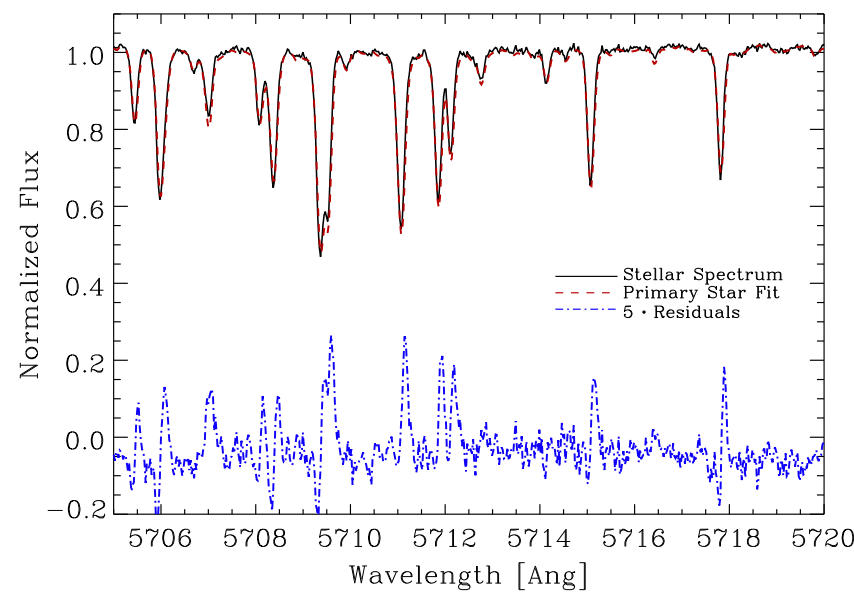

Figure 4. Best-fit to the primary star in the HD 16702 spectrum. We search through $\sim 640$ library stars with a range of $T_{\text {eff }}, \log g$, and metallicities, as well as vary the Doppler broadening and the dilution of absorption lines to correct for the finite grid spacing of the library spectra's properties. Best fits are shown as red dashed lines. After the best-fit spectrum has been identified, we subtract it from the spectrum and obtain the residuals, shown as a dotted-dashed blue line.

the Doppler shift of the studied star, as described in Section 3.1. As mentioned in Section 3.1.1, the actual $\chi^{2}$ values are of no significance. Therefore, we normalize the $\chi^{2}$ function such that its median value is one when the lines of the NSO Solar spectrum and studied spectrum are misaligned. Deviations from unity are caused by the accidental alignments of absorption lines with those corresponding to a different element.

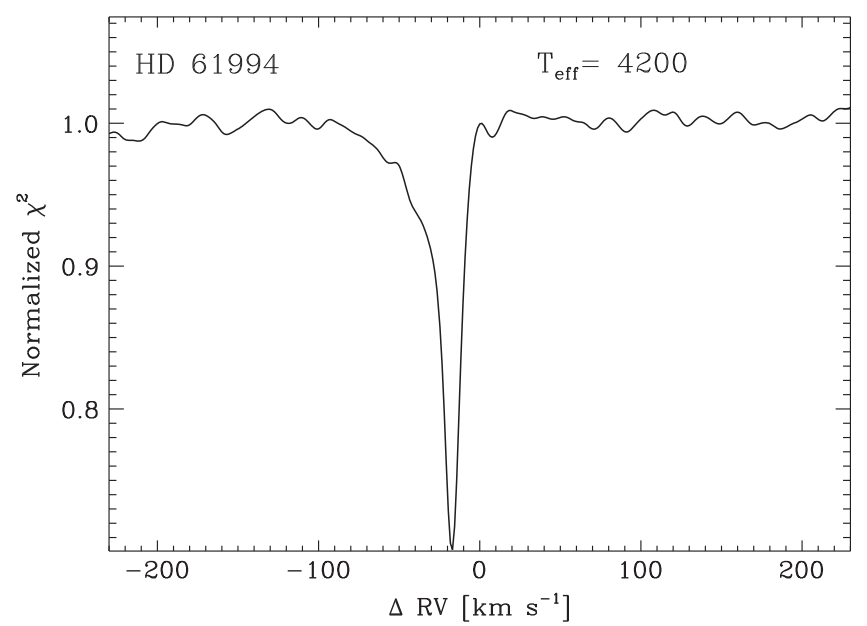

Figure 5. $\chi^{2}$ as a function of Doppler shift for the residuals of HD 61994 and the median library spectrum with $T_{\text {eff }}=4200 \mathrm{~K}$, printed in the upper right corner. There is a significant minimum at $\Delta \mathrm{RV}=-18 \mathrm{~km} \mathrm{~s}^{-1}$, indicating the presence of a second star.

We noticed a trend in those accidental alignments that was independent of the spectral type for the primary star, as all of the primary $\chi^{2}$ functions had the same shape outside the central minimum. We thus created a $\chi^{2}$ function by cross-correlating the Sun's spectrum with itself, and adopted this shape as the characteristic single star $\chi^{2}$ function. We then compare the actual $\chi^{2}$ function to this characteristic single star $\chi^{2}$ function in order to detect any discrepancies with might indicate the presence of a companion, as shown in Figure 3. 


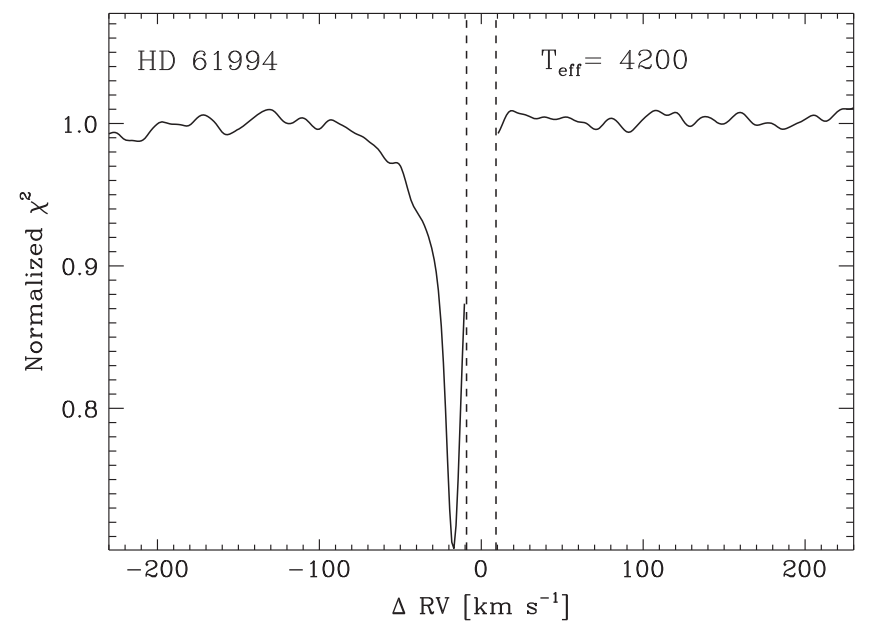

Figure 6. $\chi^{2}$ as a function of Doppler shift for the residuals of HD 61994 and the median $4200 \mathrm{~K}$ library spectrum. We mask out the central region of \pm 10 $\mathrm{km} \mathrm{s}^{-1}$, as the detection of secondary stars with relative radial velocities less than $10 \mathrm{~km} \mathrm{~s}^{-1}$ is limited. This is both due to the overlap of the two sets of lines for $|\mathrm{RV}|$ separations of less than $10 \mathrm{~km} \mathrm{~s}^{-1}$, as well as the imperfect subtraction of the primary star. Since the fit for the primary is never exact due to intrinsic spectral differences between the studied spectra and the library spectra, the residual primary star's absorption lines cause a small peak/dip at $\Delta \mathrm{RV}=0$ $\mathrm{km} \mathrm{s}^{-1}$. We cannot differentiate between the residual primary minimum and the actual secondary star, thus we exclude the central region.

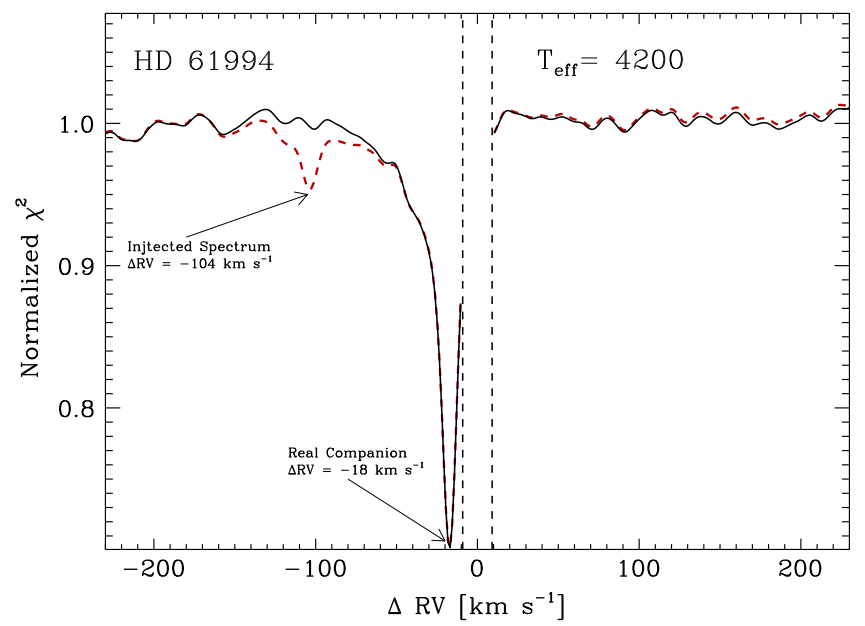

Figure 7. Residual $\chi^{2}$ function of HD 61994 and the median $4200 \mathrm{~K}$ library spectrum. The red line dashed is obtained by injecting a secondary star with $\Delta \mathrm{RV}=-104 \mathrm{~km} \mathrm{~s}^{-1}$ into the original HD 61994 spectrum. $\chi^{2}$ shown in black is the original residual $\chi^{2}$, without the injected secondary spectrum (same as Figure 5). We can see that there is a minimum at the location of the injected spectrum, annotated with an arrow. The spectrum of the injected secondary star is scaled down such that it contributes $1 \%$ of the total flux.

Table 1

Stellar Parameters for Injected SpecMatch Library Spectra

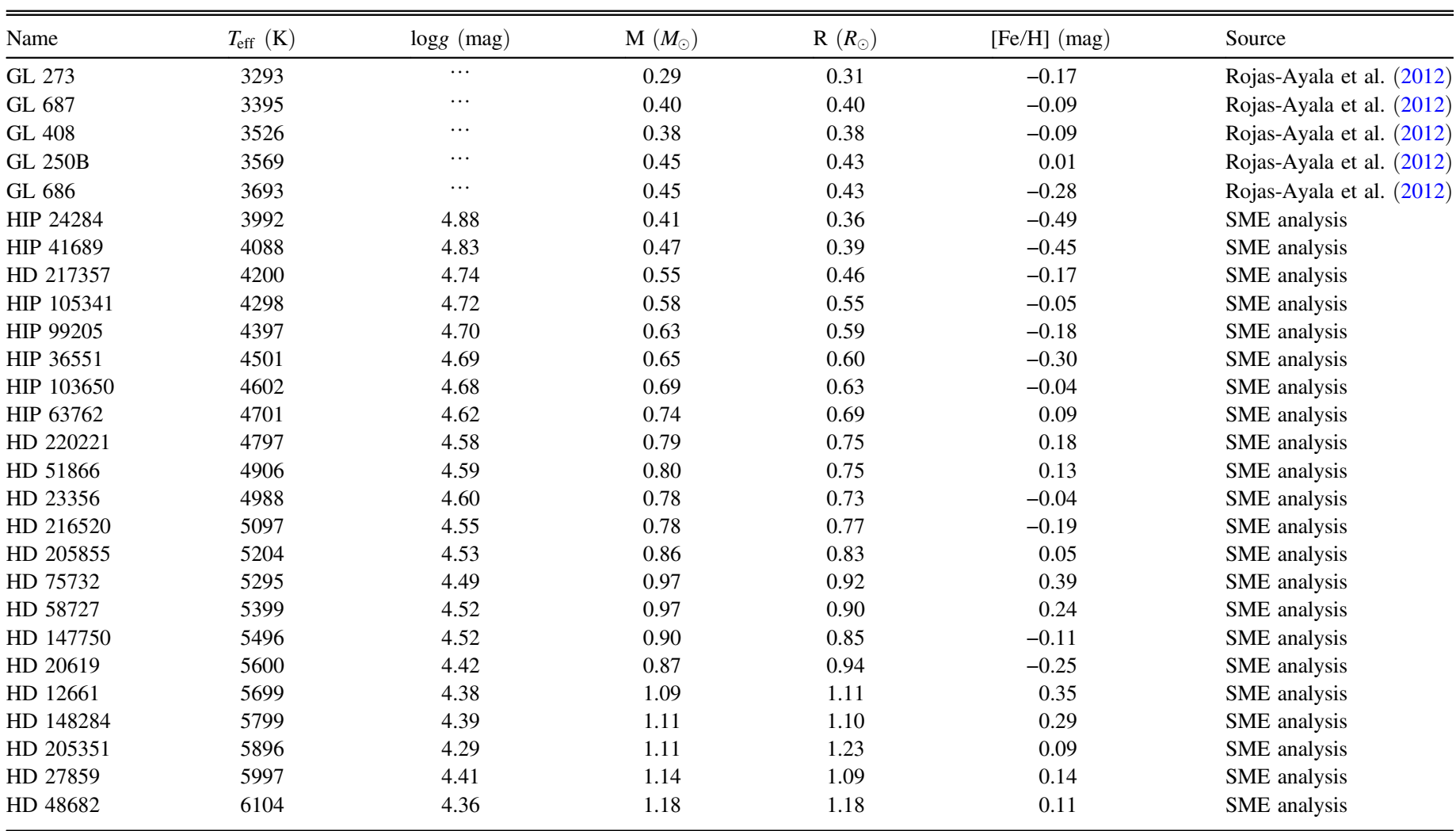

Note. No precise $\log g$ values are available for M dwarfs. 


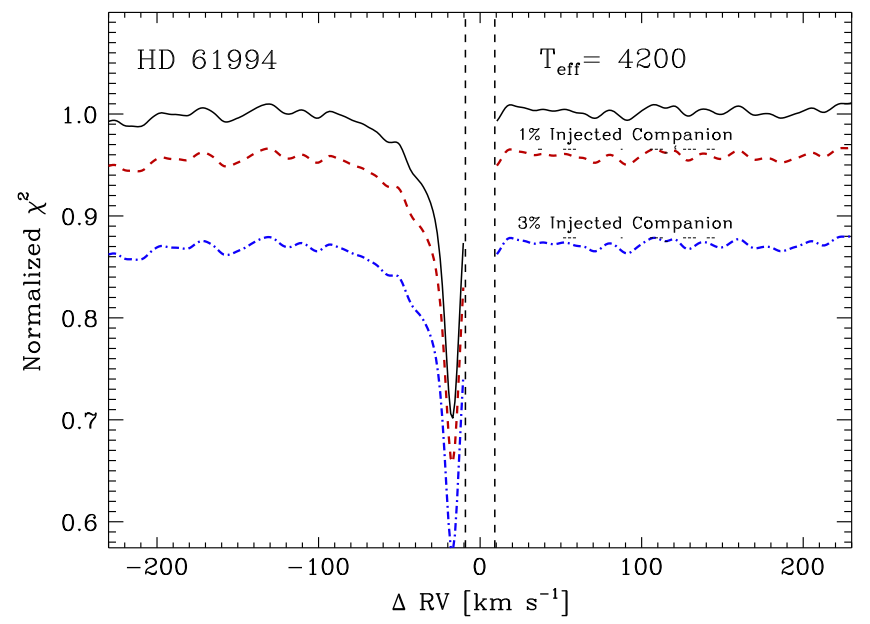

Figure 8. Residual $\chi^{2}$ functions for HD 61994 and the median $4200 \mathrm{~K}$ library spectrum. Black curve: original residual $\chi^{2}$ function without any injected secondary star. Dashed red curve: $\chi^{2}$ minima values for a secondary star that contributes $1 \%$ of the total flux. Dotted-dashed blue curve: $\chi^{2}$ minima values for a secondary star that contributes $3 \%$ of the total flux. For both dashed red and dotted-dashed blue curves, the value at each $\Delta \mathrm{RV}$ represents the value that the original residual $\chi^{2}$ would have if there was an actual secondary star present at that particular $\Delta \mathrm{RV}$ of a relative brightness indicated on the curve.

We can see in Figure 3 that the primary $\chi^{2}$ function reveals a secondary star of KIC 5473556 (at left), at a relative RV of +90 $\mathrm{km} \mathrm{s}^{-1}$. Such detections are only possible when the secondary star is sufficiently bright with a large enough relative velocity. The $\chi^{2}$ dip has a FWHM of approximately $20 \mathrm{~km} \mathrm{~s}^{-1}$, thus any secondary star with a $\Delta \mathrm{RV}<10 \mathrm{~km} \mathrm{~s}^{-1}$ would blend in and remain undetectable. Due to variable rotational broadening of the lines among different spectra, we could artificially broaden the characteristic single star $\chi^{2}$ function to match the width of the actual $\chi^{2}$ for the analyzed spectrum. Thus, the detection threshold of the relative brightness for the secondary star varies both with $\Delta \mathrm{RV}$ and the nature of the star's spectrum.

\subsection{Faint Secondary Stars}

\subsubsection{Subtraction and the Residuals}

Once the best-fit library spectrum is identified and its lines are both broadened and diluted to match the primary star's absorption lines, we subtract that best-fit library spectrum from the original spectrum, leaving residuals as shown in Figure 4.

\subsubsection{Evidence of Secondary Lines}

We re-normalize the residuals described above back to unity, and search for the secondary set of absorption lines. Our algorithm is focused mostly on faint secondary stars, so the spectral differences among two stars with $\Delta T_{\text {eff }}<100 \mathrm{~K}$ are almost indiscernible. To enhance efficiency, we create 26 median spectra with $T_{\text {eff }}$ intervals of $100 \mathrm{~K}$ starting at $3300 \mathrm{~K}$ and up to $6100 \mathrm{~K}$, rather than utilizing each individual library spectrum.

We omit $T_{\text {eff }}$ where there are less than three spectra per $100 \mathrm{~K}$ temperature interval. Consequently, not all of the $100 \mathrm{~K}$ temperature intervals are represented. In particular, we do not have a representative median library spectrum at effective temperatures 3800 and $3900 \mathrm{~K}$.

We then calculate $\chi^{2}$ as a function of Doppler shift between the residuals and each median library spectrum, and normalize it such that the median is 1 . A significant $\chi^{2}$ minimum occurring at the same $\Delta \mathrm{RV}$ for several neighboring effective temperatures of the median library spectrum indicates a possible second star in the spectrum. An example of a residual $\chi^{2}$ function is shown in Figure 5.

Unless the primary and the secondary spectrum are sufficiently different, we cannot detect secondary stars with $\Delta \mathrm{RV}<10 \mathrm{~km} \mathrm{~s}^{-1}$ relative to the primary star, due to the
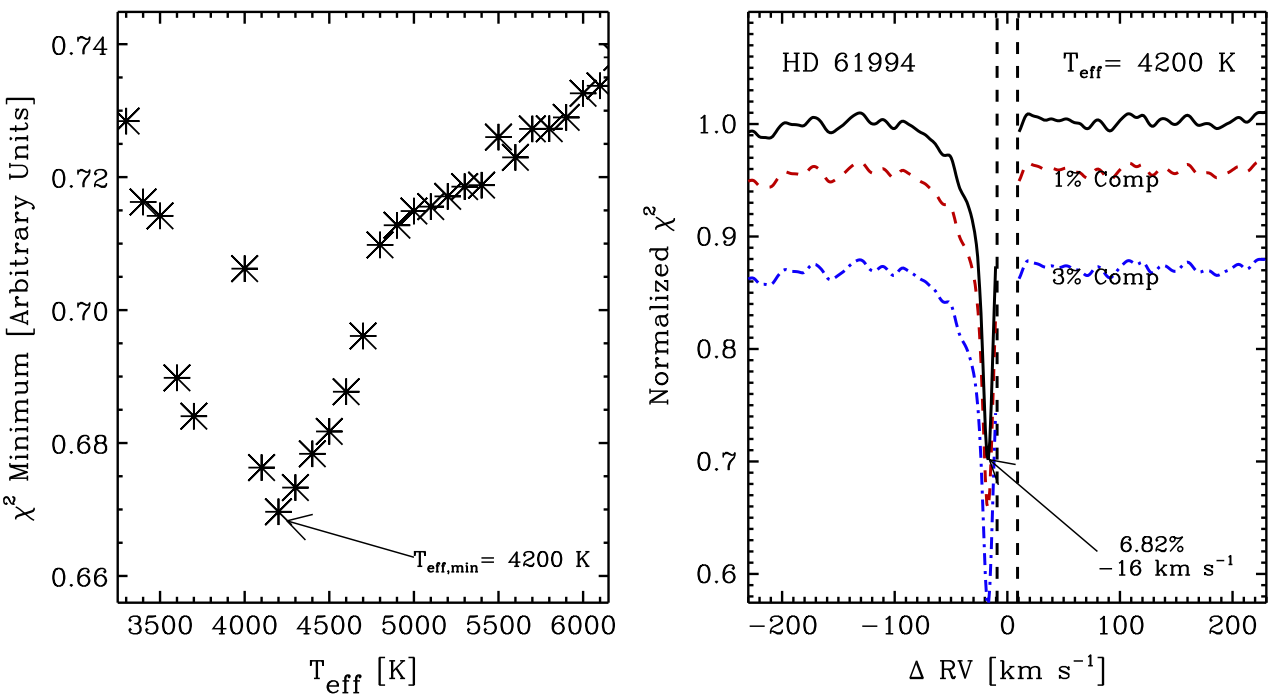

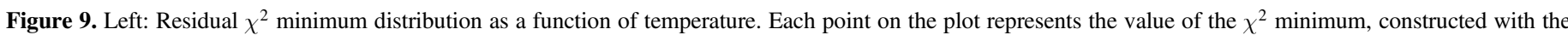

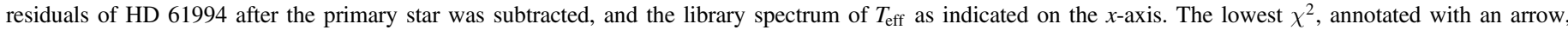

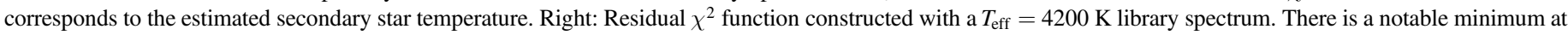

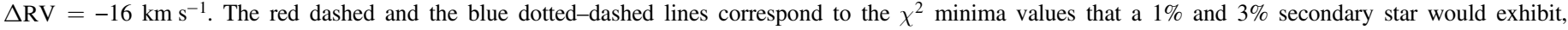

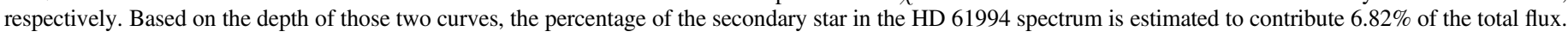




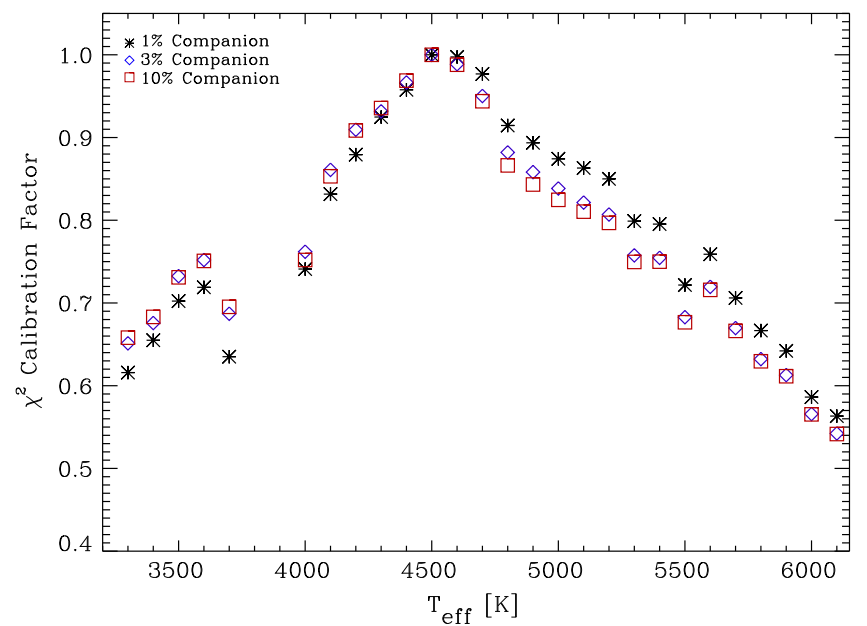

Figure 10. Calibration functions for a $1 \%, 3 \%$, and a $10 \%$ secondary star, as shown on the plot. These calibration functions were obtained by calculating the mean residual $\chi^{2}$ minimum distribution functions over all possible $T_{\text {eff }}$ secondaries. If the $\chi^{2}$ was unbiased against the temperature of the superimposed spectrum, the calibration function would be constant.

Table 2

Percentage Recovery Rates for Injection-recovery Experiment

\begin{tabular}{ccccccc}
\hline \hline \multirow{2}{*}{ Primary $T_{\text {eff }}$} & \multicolumn{5}{c}{ Secondary $T_{\text {eff }}(\mathrm{K})$} \\
\cline { 2 - 7 } & 3500 & 4000 & 4500 & 5000 & 5500 & 6000 \\
\hline \multirow{5}{*}{3500} & $50 \%$ & $40 \%$ & $40 \%$ & $60 \%$ & $60 \%$ & $60 \%$ \\
4000 & $70 \%$ & $70 \%$ & $70 \%$ & $70 \%$ & $70 \%$ & $70 \%$ \\
4500 & $80 \%$ & $70 \%$ & $80 \%$ & $80 \%$ & $70 \%$ & $70 \%$ \\
5000 & $80 \%$ & $80 \%$ & $70 \%$ & $70 \%$ & $80 \%$ & $70 \%$ \\
5500 & $100 \%$ & $90 \%$ & $90 \%$ & $100 \%$ & $100 \%$ & $80 \%$ \\
6000 & $80 \%$ & $70 \%$ & $80 \%$ & $70 \%$ & $80 \%$ & $80 \%$ \\
& & & $3 \%$ Secondary & & \\
3500 & $100 \%$ & $90 \%$ & $100 \%$ & $100 \%$ & $100 \%$ & $100 \%$ \\
4000 & $90 \%$ & $90 \%$ & $90 \%$ & $90 \%$ & $90 \%$ & $90 \%$ \\
4500 & $100 \%$ & $100 \%$ & $100 \%$ & $100 \%$ & $100 \%$ & $90 \%$ \\
5000 & $90 \%$ & $100 \%$ & $100 \%$ & $100 \%$ & $90 \%$ & $90 \%$ \\
5500 & $100 \%$ & $100 \%$ & $100 \%$ & $100 \%$ & $100 \%$ & $100 \%$ \\
6000 & $90 \%$ & $100 \%$ & $100 \%$ & $100 \%$ & $90 \%$ & $90 \%$ \\
& & \multicolumn{5}{c}{$10 \%$ Secondary } \\
3500 & $100 \%$ & $100 \%$ & $100 \%$ & $100 \%$ & $100 \%$ & $100 \%$ \\
4000 & $100 \%$ & $100 \%$ & $100 \%$ & $100 \%$ & $100 \%$ & $100 \%$ \\
4500 & $100 \%$ & $100 \%$ & $100 \%$ & $100 \%$ & $100 \%$ & $100 \%$ \\
5000 & $100 \%$ & $100 \%$ & $100 \%$ & $100 \%$ & $100 \%$ & $100 \%$ \\
5500 & $100 \%$ & $100 \%$ & $100 \%$ & $100 \%$ & $100 \%$ & $100 \%$ \\
6000 & $100 \%$ & $100 \%$ & $100 \%$ & $100 \%$ & $100 \%$ & $100 \%$ \\
\hline
\end{tabular}

normal widths of the absorption lines. None of the library spectra are the exact match to the studied ones, so there will always be some imperfections in the best-fitting library star, resulting in larger residuals at the locations of primary star's absorption lines. These residuals may cause either small peak or a small dip at $\Delta \mathrm{RV}=0 \mathrm{~km} \mathrm{~s}^{-1}$. Thus, if both stars have exactly the same radial velocity, we cannot differentiate between the minimum at $\Delta \mathrm{RV}=0 \mathrm{~km} \mathrm{~s}^{-1}$ that is due to the imperfect subtraction of the primary, or that due to an actual second star. Furthermore, for secondary sets of absorption lines with slightly different radial velocities than the primary star, their spectral lines are still blended with the primary star's absorption lines. This occurs if the RV separation is less than $10 \mathrm{~km} \mathrm{~s}^{-1}$, which is the typical width of an absorption line. This blending causes a fraction of secondary absorption lines to be subtracted away together with the primary star, making a faint second star appear even fainter, and in most cases impossible to detect.

Due to the limitations described, we mask out the central region with $\Delta \mathrm{RV}<10 \mathrm{~km} \mathrm{~s}^{-1}$, as shown in Figure 6. The only exception is the case where the two spectral types are sufficiently different that the primary star spectrum does not interfere with the secondary, regardless of their RV separation. This is the case of a G-type primary and an M dwarf secondary star, as further discussed in Section 4.1.4.

\subsubsection{Estimating Secondary Star Temperature}

As mentioned in Section 3.3.2, we obtain 26 distinct residual $\chi^{2}$ functions, differing in the effective temperature of the library spectrum superimposed with the studied residual spectrum. We expect to find a unique $\Delta \mathrm{RV}$ location of the secondary star, if one is detectable. Because a secondary set of absorption lines would cause a minimum in $\chi^{2}$ over a range of library spectral types that are close in $T_{\text {eff }}$ to the actual temperature, we assume the secondary star $\Delta \mathrm{RV}$ to be the mode of the $\chi^{2}$ minimum locations for a set of all 29 residual $\chi^{2}$ functions. The exception here is the case where the minimum occurs only for $T_{\text {eff }} \sim 3500 \mathrm{~K}$. Due to a significantly different $\mathrm{M}$ dwarf spectrum, it is possible that the secondary star is detected only in a few residual $\chi^{2}$ functions with $T_{\text {eff }}<\sim 3800 \mathrm{~K}$. Those cases are examined and assessed on an individual basis.

Using the mode minimum $\Delta \mathrm{RV}$ location, we plot the $\chi^{2}$ values at those locations versus the temperature of the median library spectrum used to construct each respective $\chi^{2}$ function. For most residual $\chi^{2}$ functions, this $\chi^{2}$ is indeed the $\chi^{2}$ minimum. Ideally, the depth of the minimum should only depend on how well the library spectrum correlates with the spectrum of the secondary star, producing the lowest $\chi^{2}$ minimum when the library spectrum temperature is closest to the effective temperature of the secondary star. However, mostly due to the low $\mathrm{S} / \mathrm{N}$ of the residuals, we noticed a dependence of the residual $\chi^{2}$ minimum on the temperature of the library spectrum that was independent of the actual $T_{\text {eff }}$ of the secondary star. In order to correct for this, we divide the $\chi^{2}$ minima plot by the calibration function, a construction of which is described in more detail in Section 4.1.2.

We then plot the calibrated $\chi^{2}$ minimum values versus the temperature of the median library spectrum, as shown in Figure 9 (left). In order to determine approximate temperature of the secondary star, we follow the same procedure as for the primary star, as outlined in Section 3.1.1. In brief, we fit the points with a polynomial, find a minimum, remove the outliers that differ from the fit by more than $3 \sigma$, re-fit the remaining points, and restrict the possible range of secondary star $T_{\text {eff }}$ to \pm $200 \mathrm{~K}$ around the polynomial minimum. Within that range, we then compute the mean $T_{\text {eff }}$ of the three median library spectra corresponding to the three lowest $\chi^{2}$ minima. We adopt this mean as the estimated temperature of the secondary star. Taking the mean of the three lowest $T_{\text {eff }}$ values corrects for the missing median templates, because not every $100 \mathrm{~K} T_{\text {eff }}$ is represented due to the varying parameter populations of SpecMatch library. 
Table 3

Systematic Errors and Uncertainties for the Estimated Secondary Star Temperature

\begin{tabular}{|c|c|c|c|c|c|c|}
\hline \multirow{2}{*}{ Primary $T_{\text {eff }}(\mathrm{K})$} & \multicolumn{6}{|c|}{ Secondary $T_{\text {eff }}(\mathrm{K})$} \\
\hline & 3500 & 4000 & 4500 & 5000 & 5500 & 6000 \\
\hline & \multicolumn{6}{|c|}{$1 \%$ Secondary } \\
\hline 3500 & $75 \pm 70$ & $565 \pm 200$ & $30 \pm 695$ & $-245 \pm 415$ & $45 \pm 465$ & $500 \pm 465$ \\
\hline 4000 & $-45 \pm 155$ & $175 \pm 440$ & $-55 \pm 530$ & $-470 \pm 440$ & $-230 \pm 320$ & $180 \pm 330$ \\
\hline 4500 & $0 \pm 125$ & $195 \pm 455$ & $80 \pm 220$ & $-285 \pm 260$ & $-355 \pm 70$ & $115 \pm 180$ \\
\hline 5000 & $-85 \pm 155$ & $500 \pm 125$ & $230 \pm 135$ & $-205 \pm 230$ & $-14- \pm 390$ & $50 \pm 20$ \\
\hline 5500 & $5 \pm 155$ & $230 \pm 440$ & $140 \pm 235$ & $-260 \pm 235$ & $-375 \pm 95$ & $60 \pm 25$ \\
\hline \multirow[t]{2}{*}{6000} & $-85 \pm 155$ & $500 \pm 125$ & $230 \pm 125$ & $-205 \pm 230$ & $-140 \pm 390$ & $50 \pm 20$ \\
\hline & \multicolumn{6}{|c|}{$3 \%$ Secondary } \\
\hline 3500 & $-60 \pm 185$ & $470 \pm 340$ & $200 \pm 355$ & $-85 \pm 410$ & $-180 \pm 360$ & $85 \pm 65$ \\
\hline 4000 & $-5 \pm 130$ & $380 \pm 420$ & $195 \pm 325$ & $-80 \pm 480$ & $-255 \pm 440$ & $205 \pm 450$ \\
\hline 4500 & $25 \pm 135$ & $385 \pm 370$ & $250 \pm 350$ & $-195 \pm 295$ & $-355 \pm 145$ & $50 \pm 150$ \\
\hline 5000 & $0 \pm 135$ & $80 \pm 370$ & $250 \pm 250$ & $-210 \pm 385$ & $225 \pm 435$ & $330 \pm 495$ \\
\hline 5500 & $-5 \pm 160$ & $375 \pm 255$ & $225 \pm 255$ & $-220 \pm 320$ & $-375 \pm 105$ & $50 \pm 150$ \\
\hline \multirow[t]{2}{*}{6000} & $0 \pm 135$ & $80 \pm 375$ & $250 \pm 250$ & $-210 \pm 370$ & $205 \pm 435$ & $330 \pm 495$ \\
\hline & \multicolumn{6}{|c|}{$10 \%$ Secondary } \\
\hline 3500 & $-15 \pm 145$ & $295 \pm 170$ & $22 \pm 185$ & $70 \pm 245$ & $-155 \pm 345$ & $-50 \pm 50$ \\
\hline 4000 & $-5 \pm 130$ & $250 \pm 120$ & $-50 \pm 220$ & $-15 \pm 275$ & $-230 \pm 280$ & $-50 \pm 50$ \\
\hline 4500 & $-10 \pm 130$ & $205 \pm 135$ & $100 \pm 50$ & $-20 \pm 315$ & $-195 \pm 320$ & $100 \pm 14$ \\
\hline 5000 & $0 \pm 150$ & $205 \pm 85$ & $25 \pm 150$ & $30 \pm 255$ & $-145 \pm 245$ & $-20 \pm 50$ \\
\hline 5500 & $-5 \pm 150$ & $190 \pm 160$ & $130 \pm 65$ & $-95 \pm 345$ & $-320 \pm 125$ & $-50 \pm 20$ \\
\hline 6000 & $0 \pm 150$ & $300 \pm 80$ & $150 \pm 100$ & $30 \pm 255$ & $-145 \pm 245$ & $-40 \pm 50$ \\
\hline
\end{tabular}

Table 4

Systematic Errors and Uncertainties for the Estimated Percentage Flux of the Secondary Star

\begin{tabular}{|c|c|c|c|c|c|c|}
\hline \multirow{2}{*}{ Primary $T_{\text {eff }}(\mathrm{K})$} & \multicolumn{6}{|c|}{ Secondary $T_{\text {eff }}(\mathrm{K})$} \\
\hline & 3500 & 4000 & 4500 & 5000 & 5500 & 6000 \\
\hline & \multicolumn{6}{|c|}{$1 \%$ Secondary } \\
\hline 3500 & $0.25 \pm 0.10$ & $-0.28 \pm 0.89$ & $0.03 \pm 0.33$ & $-0.30 \pm 0.24$ & $-0.21 \pm 0.29$ & $-0.15 \pm 0.34$ \\
\hline 4000 & $0.21 \pm 0.11$ & $0.20 \pm 0.26$ & $-0.01 \pm 0.11$ & $-0.18 \pm 0.24$ & $-0.10 \pm 0.18$ & $-0.04 \pm 0.21$ \\
\hline 4500 & $0.10 \pm 0.26$ & $0.11 \pm 0.26$ & $-0.07 \pm 0.12$ & $-0.25 \pm 0.34$ & $-0.16 \pm 0.11$ & $-0.14 \pm 0.16$ \\
\hline 5000 & $0.32 \pm 0.19$ & $0.32 \pm 0.19$ & $0.03 \pm 0.19$ & $-0.04 \pm 0.25$ & $-0.02 \pm 0.17$ & $-0.05 \pm 0.22$ \\
\hline 5500 & $0.15 \pm 0.18$ & $0.11 \pm 0.26$ & $-0.11 \pm 0.17$ & $-0.12 \pm 0.20$ & $-0.23 \pm 0.31$ & $-0.07 \pm 0.14$ \\
\hline \multirow[t]{2}{*}{6000} & $0.32 \pm 0.18$ & $0.32 \pm 0.19$ & $0.03 \pm 0.19$ & $-0.04 \pm 0.25$ & $-0.02 \pm 0.17$ & $-0.05 \pm 0.22$ \\
\hline & \multicolumn{6}{|c|}{$3 \%$ Secondary } \\
\hline 3500 & $0.34 \pm 0.90$ & $0.67 \pm 0.45$ & $0.00 \pm 0.40$ & $-0.21 \pm 0.66$ & $-0.34 \pm 0.70$ & $-0.35 \pm 0.74$ \\
\hline 4000 & $0.32 \pm 0.43$ & $0.71 \pm 0.65$ & $-0.08 \pm 0.32$ & $-0.23 \pm 0.57$ & $-0.24 \pm 0.78$ & $0.19 \pm 0.69$ \\
\hline 4500 & $0.38 \pm 0.43$ & $0.62 \pm 0.50$ & $0.07 \pm 0.69$ & $-0.25 \pm 0.50$ & $-0.31 \pm 0.52$ & $-0.02 \pm 0.34$ \\
\hline 5000 & $0.31 \pm 0.86$ & $0.11 \pm 0.79$ & $-0.12 \pm 0.23$ & $-0.18 \pm 0.18$ & $-0.11 \pm 0.14$ & $-0.49 \pm 0.16$ \\
\hline 5500 & $0.25 \pm 0.49$ & $0.67 \pm 0.49$ & $-0.21 \pm 0.38$ & $-0.18 \pm 0.42$ & $-0.45 \pm 0.53$ & $0.04 \pm 0.29$ \\
\hline \multirow[t]{2}{*}{6000} & $0.18 \pm 0.87$ & $0.11 \pm 0.79$ & $-0.12 \pm 0.23$ & $0.18 \pm 0.18$ & $0.11 \pm 0.14$ & $0.69 \pm 0.16$ \\
\hline & \multicolumn{6}{|c|}{$10 \%$ Secondary } \\
\hline 3500 & $1.17 \pm 1.34$ & $3.09 \pm 1.21$ & $0.35 \pm 1.85$ & $0.06 \pm 2.25$ & $-2.63 \pm 2.50$ & $-1.17 \pm 1.42$ \\
\hline 4000 & $1.26 \pm 1.40$ & $3.43 \pm 1.09$ & $0.23 \pm 1.40$ & $-1.92 \pm 1.44$ & $-1.84 \pm 0.77$ & $-0.68 \pm 1.44$ \\
\hline 4500 & $1.32 \pm 1.55$ & $2.95 \pm 0.77$ & $-0.12 \pm 1.05$ & $-1.26 \pm 0.83$ & $-1.97 \pm 1.08$ & $-1.07 \pm 1.97$ \\
\hline 5000 & $-0.07 \pm 0.15$ & $2.23 \pm 1.43$ & $0.29 \pm 0.70$ & $-1.25 \pm 0.93$ & $-1.76 \pm 0.83$ & $-1.55 \pm 1.53$ \\
\hline 5500 & $0.55 \pm 1.56$ & $2.00 \pm 1.00$ & $-0.84 \pm 0.78$ & $-1.71 \pm 0.41$ & $-1.71 \pm 0.53$ & $-0.47 \pm 0.50$ \\
\hline 6000 & $-0.07 \pm 0.16$ & $2.23 \pm 1.43$ & $0.29 \pm 0.70$ & $-0.42 \pm 0.58$ & $-1.76 \pm 0.83$ & $-1.55 \pm 1.53$ \\
\hline
\end{tabular}

We cannot extrapolate the behavior of the $\chi^{2}$ minima function to values of $T_{\text {eff }}$ higher than $6100 \mathrm{~K}$ or lower than $3300 \mathrm{~K}$. Thus, any secondary stars whose $T_{\text {eff }}$ is identified at $\sim 6000 \mathrm{~K}$, is not guaranteed to be at that temperature; rather this serves as the lower temperature limit, as the secondary star can be hotter than $\sim 6000 \mathrm{~K}$ but not cooler. Similarly, secondary stars whose estimated $T_{\text {eff }}$ is $3300 \mathrm{~K}$ can be cooler than this value, but not hotter. This is discussed more in detail in Section 6.2.

\subsubsection{Estimating Relative Brightness}

After determining $T_{\text {eff }}$ of the second star, we use the corresponding residual $\chi^{2}$ function for further analysis. We aim to estimate the brightness of the secondary star relative to the 
Table 5

Uncertainties in the Effective Temperature of the Secondary Star

\begin{tabular}{|c|c|c|c|c|c|c|c|c|c|c|c|c|}
\hline \multirow[t]{3}{*}{ Primary $T_{\text {eff }}(\mathrm{K})$} & \multicolumn{12}{|c|}{ Secondary $T_{\text {eff }}(\mathrm{K})$} \\
\hline & 3500 & 4000 & 4500 & 5000 & 5500 & 6000 & 3500 & 4000 & 4500 & 5000 & 5500 & 6000 \\
\hline & \multicolumn{6}{|c|}{ Absolute $T_{\text {eff }}$ Uncertainty, $\sigma_{T_{\mathrm{eff}}}(\mathrm{K})$} & \multicolumn{6}{|c|}{ Relative Flux Ratio Uncertainty ${ }^{\mathrm{a}}, \sigma_{f} / f$} \\
\hline & \multicolumn{6}{|c|}{$1 \%$ Secondary } & \multicolumn{6}{|c|}{$1 \%$ Secondary } \\
\hline 3500 & 150 & 750 & 750 & 650 & 500 & 950 & 0.35 & 1.15 & 0.35 & 0.55 & 0.50 & 0.50 \\
\hline 4000 & 200 & 600 & 600 & 900 & 550 & 500 & 0.30 & 0.45 & 0.10 & 0.40 & 0.30 & 0.25 \\
\hline 4500 & 150 & 650 & 300 & 550 & 450 & 300 & 0.35 & 0.35 & 0.20 & 0.60 & 0.25 & 0.30 \\
\hline 5000 & 250 & 650 & 350 & 450 & 400 & 100 & 0.50 & 0.50 & 0.20 & 0.30 & 0.20 & 0.30 \\
\hline 5500 & 150 & 650 & 400 & 500 & 450 & 100 & 0.35 & 0.35 & 0.30 & 0.30 & 0.55 & 0.20 \\
\hline \multirow[t]{2}{*}{6000} & 250 & 650 & 350 & 450 & 550 & 100 & 0.50 & 0.50 & 0.20 & 0.30 & 0.20 & 0.25 \\
\hline & \multicolumn{6}{|c|}{$3 \%$ Secondary } & \multicolumn{6}{|c|}{$3 \%$ Secondary } \\
\hline 3500 & 250 & 800 & 550 & 500 & 550 & 150 & 0.40 & 0.35 & 0.15 & 0.30 & 0.35 & 0.35 \\
\hline 4000 & 150 & 800 & 500 & 550 & 700 & 650 & 0.25 & 0.45 & 0.15 & 0.25 & 0.35 & 0.30 \\
\hline 4500 & 150 & 755 & 600 & 500 & 500 & 200 & 0.25 & 0.35 & 0.25 & 0.25 & 0.25 & 0.10 \\
\hline 5000 & 150 & 450 & 500 & 600 & 650 & 850 & 0.40 & 0.30 & 0.10 & 0.10 & 0.10 & 0.20 \\
\hline 5500 & 150 & 650 & 500 & 550 & 500 & 200 & 0.25 & 0.40 & 0.20 & 0.20 & 0.30 & 0.10 \\
\hline \multirow[t]{2}{*}{6000} & 150 & 450 & 500 & 600 & 650 & 850 & 0.35 & 0.30 & 0.10 & 0.10 & 0.10 & 0.30 \\
\hline & \multicolumn{6}{|c|}{$10 \%$ Secondary } & \multicolumn{6}{|c|}{$10 \%$ Secondary } \\
\hline 3500 & 150 & 450 & 200 & 300 & 550 & 100 & 0.25 & 0.45 & 0.20 & 0.25 & 0.50 & 0.25 \\
\hline 4000 & 150 & 350 & 250 & 350 & 500 & 100 & 0.25 & 0.45 & 0.15 & 0.35 & 0.25 & 0.20 \\
\hline 4500 & 150 & 350 & 150 & 350 & 500 & 100 & 0.30 & 0.35 & 0.10 & 0.20 & 0.30 & 0.30 \\
\hline 5000 & 150 & 300 & 200 & 300 & 400 & 100 & 0.10 & 0.35 & 0.10 & 0.20 & 0.25 & 0.30 \\
\hline 5500 & 150 & 350 & 200 & 450 & 450 & 100 & 0.20 & 0.30 & 0.15 & 0.20 & 0.20 & 0.10 \\
\hline 6000 & 150 & 400 & 250 & 300 & 400 & 100 & 0.05 & 0.35 & 0.10 & 0.10 & 0.25 & 0.30 \\
\hline
\end{tabular}

Note.

a The flux ratio of the secondary and primary star is denoted by $f$, where $f=F_{B} / F_{A}$.

Table 6

Injection-recovery Experiment: Recovery Rates and Parameter Uncertainties for a G-type Primary Star and an M dwarf Secondary at $+5 \mathrm{~km} \mathrm{~s}^{-1} \Delta \mathrm{RV}$

\begin{tabular}{cccc}
\hline \hline $\begin{array}{c}\text { Secondary Star } \\
\text { Brightness }\end{array}$ & Recovery & $\begin{array}{c}T_{\text {eff }}(\mathrm{K}) \\
T_{\text {actual }}-T_{\text {deduced }}\end{array}$ & $\begin{array}{c}\text { Relative Flux }(\%) \\
\%_{\text {actual }}-\%_{\text {deduced }}\end{array}$ \\
\hline $1 \%$ & Rate & $35 \pm 170$ & $0.01 \pm 0.22$ \\
$3 \%$ & $40 \%$ & $10 \pm 130$ & $1.18 \pm 0.56$ \\
$5 \%$ & $90 \%$ & $-5 \pm 140$ & $2.05 \pm 0.70$ \\
\hline
\end{tabular}

primary star based on the depth of that residual $\chi^{2}$ function minimum.

We do so by synthesizing the effect of secondary spectra. We inject another spectrum into the original studied spectrum. We scale down the injected spectrum such that it contributes either 3\% or $1 \%$ of the total flux, and shift its absorption lines to a known relative radial velocity. The injected spectrum has $T_{\text {eff }}$ close to that already estimated for the secondary star temperature, assuring that the superimposed library spectrum fits both the actual secondary star and the injected spectrum equally well. The properties of all the possible injected spectra are listed in Table 1.

We then treat this original spectrum, with its synthetic injected secondary star spectrum, as the new studied spectrum, and repeat the whole procedure outlined in Sections 3.1.1 through 3.3.2. We record the $\chi^{2}$ value at the $\Delta \mathrm{RV}$ location of the injected spectrum, as annotated in Figure 7. At that particular $\Delta \mathrm{RV}$, the difference between the injected spectrum $\chi^{2}$ and the value of the original $\chi^{2}$, both shown in Figure 7 , represents the minimum depth that would be caused by a secondary star of the injected brightness.
To obtain a more statistically useful sample of possible secondary spectra, we repeat the injection for several different values $\Delta \mathrm{RVs}$, and record the difference between the value of the $\chi^{2}$ minimum at the location of the injected secondary spectrum and the original residual $\chi^{2}$ value at that location. Using those differences, we then calculate the median $\chi^{2}$ minimum depth relative to the original residual $\chi^{2}$ caused by a secondary star of that particular brightness.

Subtracting the median depth for both the $3 \%$ and $1 \%$ injected secondary spectrum from the original residual $\chi^{2}$ without the injected secondary spectrum, we obtain the plot shown in Figure 8. At each $\Delta \mathrm{RV}$, the two colored curves represent the value that the $\chi^{2}$ function would have if there was a secondary star of the specified brightness located at that particular $\Delta \mathrm{RV}$. This allows for a visual comparison of the actual residual $\chi^{2}$ minimum to the characteristic $\chi^{2}$ minima depths for $3 \%$ and $1 \%$ secondary stars.

To estimate the relative brightness of the actual secondary star, we extrapolate the median $\chi^{2}$ minimum caused by the $3 \%$ and $1 \%$ injected secondary spectrum to the actual $\chi^{2}$ minimum for the residual function. We use a least-squares linear fit, with a restriction that $0 \%$ secondary star (none present) causes a $\chi^{2}$ minimum of depth 0 . We report both the $\Delta \mathrm{RV}$ of the secondary star, as well as the estimated relative brightness as a percentage of total flux, on the plot.

Since our wavelength domain encompasses approximately the $\mathrm{V}$ and $\mathrm{R}$ broadbands of the classical photometry, the relative brightness of the two stars can be used to compute the dilution factor of the Kepler light curves. Thus, if the primary star has any planet candidates, this dilution factor can help determine a more accurate radius of the planet. 
Table 7

Parameters for the Test Cases of Binary Stars; Primary Star

\begin{tabular}{|c|c|c|c|c|}
\hline Parameter & Literature & Our Results & Discrepancy & Notes \\
\hline \multicolumn{5}{|c|}{ KIC 10319590} \\
\hline$T_{\text {eff }}(K)$ & $5518 \pm 200$ & $5650 \pm 200$ & / & MAST Online Catalog \\
\hline Mass $\left(M_{\odot}\right)$ & $\cdots$ & $1.0 \pm 0.5$ & $\cdots$ & MAST Online Catalog \\
\hline Radius $\left(R_{\odot}\right)$ & $\cdots$ & $0.9 \pm 0.5$ & $\cdots$ & MAST Online Catalog \\
\hline $\log g(\mathrm{mag})$ & $4.4 \pm 0.5$ & $4.5 \pm 0.5$ & / & MAST Online Catalog \\
\hline \multicolumn{5}{|c|}{ KIC 5473556} \\
\hline$T_{\text {eff }}(\mathrm{K})$ & $5932 \pm 200$ & $5800 \pm 200$ & l & MAST Online Catalog \\
\hline $\operatorname{Mass}\left(M_{\odot}\right)$ & $\cdots$ & $1.0 \pm 0.5$ & $\cdots$ & MAST Online Catalog \\
\hline Radius $\left(R_{\odot}\right)$ & $\cdots$ & $0.9 \pm 0.5$ & $\cdots$ & MAST Online Catalog \\
\hline $\log g(\operatorname{mag})$ & $4.028 \pm 0.5$ & $4.5 \pm 0.5$ & I & MAST Online Catalog \\
\hline \multicolumn{5}{|c|}{ KIC 8572936} \\
\hline$T_{\text {eff }}(\mathrm{K})$ & $5913 \pm 130$ & $6000 \pm 200$ & / & Welsh et al. (2011) \\
\hline Mass $\left(M_{\odot}\right)$ & 1.0479 & $1.0 \pm 0.5$ & l & Welsh et al. (2011) \\
\hline Radius $\left(R_{\odot}\right)$ & 1.1618 & $1.1 \pm 0.5$ & l & Welsh et al. (2011) \\
\hline $\log g(\operatorname{mag})$ & $4.3284 \pm 0.5$ & $4.3 \pm 0.5$ & l & Welsh et al. (2011) \\
\hline \multicolumn{5}{|c|}{ KIC 9837578} \\
\hline$T_{\text {eff }}(\mathrm{K})$ & $5606 \pm 150$ & $5800 \pm 200$ & l & Welsh et al. (2011) \\
\hline Mass $\left(M_{\odot}\right)$ & 0.8877 & $0.9 \pm 0.5$ & l & Welsh et al. (2011) \\
\hline Radius $\left(R_{\odot}\right)$ & 1.0284 & $0.9 \pm 0.5$ & I & Welsh et al. (2011) \\
\hline $\log g(\operatorname{mag})$ & $4.3623 \pm 0.5$ & $4.5 \pm 0.5$ & l & Welsh et al. (2011) \\
\hline \multicolumn{5}{|c|}{ HD 61994} \\
\hline$T_{\text {eff }}(\mathrm{K})$ & $5630 \pm 150$ & $5750 \pm 200$ & / & Strassmeier et al. (2012) \\
\hline Mass $\left(M_{\odot}\right)$ & $\cdots$ & $1.1 \pm 0.5$ & $\cdots$ & $\cdots$ \\
\hline Radius $\left(R_{\odot}\right)$ & $\cdots$ & $1.3 \pm 0.5$ & $\cdots$ & $\cdots$ \\
\hline $\log g(\operatorname{mag})$ & $4.13 \pm 0.11$ & $4.2 \pm 0.5$ & I & Strassmeier et al. (2012) \\
\hline \multicolumn{5}{|c|}{ HD 16702} \\
\hline$T_{\text {eff }}(\mathrm{K})$ & $5908 \pm 25$ & $5800 \pm 200$ & I & Díaz et al. (2012) \\
\hline Mass $\left(M_{\odot}\right)$ & $0.98 \pm 0.04$ & $1.1 \pm 0.5$ & l & Díaz et al. (2012) \\
\hline Radius $\left(R_{\odot}\right)$ & $\cdots$ & $1.0 \pm 0.5$ & $\cdots$ & $\cdots$ \\
\hline $\log g(\operatorname{mag})$ & $4.46 \pm 0.03$ & $4.4 \pm 0.5$ & I & Díaz et al. (2012) \\
\hline
\end{tabular}

Table 8

Parameters for the Test Cases of Binary Stars; Stellar Companion

\begin{tabular}{|c|c|c|c|c|}
\hline Parameter & Literature & Our Results & Discrepancy & Notes \\
\hline \multicolumn{5}{|c|}{ KIC 10319590} \\
\hline$T_{\text {eff }}(\mathrm{K})$ & $\cdots$ & $4300 \pm 500$ & $\cdots$ & MAST Online Catalog \\
\hline Flux ratio, $\mathrm{F}_{B} / \mathrm{F}_{A}$ & $\cdots$ & $0.036 \pm 0.007$ & $\cdots$ & $\cdots$ \\
\hline \multicolumn{5}{|c|}{ KIC 5473556} \\
\hline$T_{\text {eff }}(\mathrm{K})$ & $\cdots$ & $6000 \pm 100$ & $\cdots$ & MAST Online Catalog \\
\hline Flux ratio, $\mathrm{F}_{B} / \mathrm{F}_{A}$ & $\cdots$ & $0.22 \pm 0.07$ & $\cdots$ & $\cdots$ \\
\hline \multicolumn{5}{|c|}{ KIC 8572936} \\
\hline$T_{\text {eff }}(\mathrm{K})$ & $5867 \pm 130$ & $6000 \pm 250$ & l & Welsh et al. (2011) \\
\hline Flux ratio, $\mathrm{F}_{B} / \mathrm{F}_{A}$ & $0.8475 \pm 0.005$ & $0.82 \pm 0.25$ & l & Welsh et al. (2011) \\
\hline \multicolumn{5}{|c|}{ KIC 9837578} \\
\hline$T_{\text {eff }}(\mathrm{K})$ & $5202 \pm 100$ & $5600 \pm 400$ & / & Strassmeier et al. (2012) \\
\hline Flux ratio, $\mathrm{F}_{B} / \mathrm{F}_{A}$ & 0.3941 & $0.56 \pm 0.14$ & $7 \%$ & Strassmeier et al. (2012) \\
\hline \multicolumn{5}{|c|}{ HD 61994} \\
\hline$T_{\text {eff }}(\mathrm{K})$ & $4775 \pm 150$ & $4200 \pm 650$ & l & Strassmeier et al. (2012) \\
\hline Flux ratio, $\mathrm{F}_{B} / \mathrm{F}_{A}$ & 0.069 & $0.055 \pm 0.022$ & l & Strassmeier et al. (2012) \\
\hline \multicolumn{5}{|c|}{ HD 16702} \\
\hline$T_{\text {eff }}(\mathrm{K})$ & $\cdots$ & $3500 \pm 250$ & $\cdots$ & $\cdots$ \\
\hline Flux ratio, $\mathrm{F}_{B} / \mathrm{F}_{A}$ & $\cdots$ & $0.016 \pm 0.008$ & $\cdots$ & $\cdots$ \\
\hline
\end{tabular}




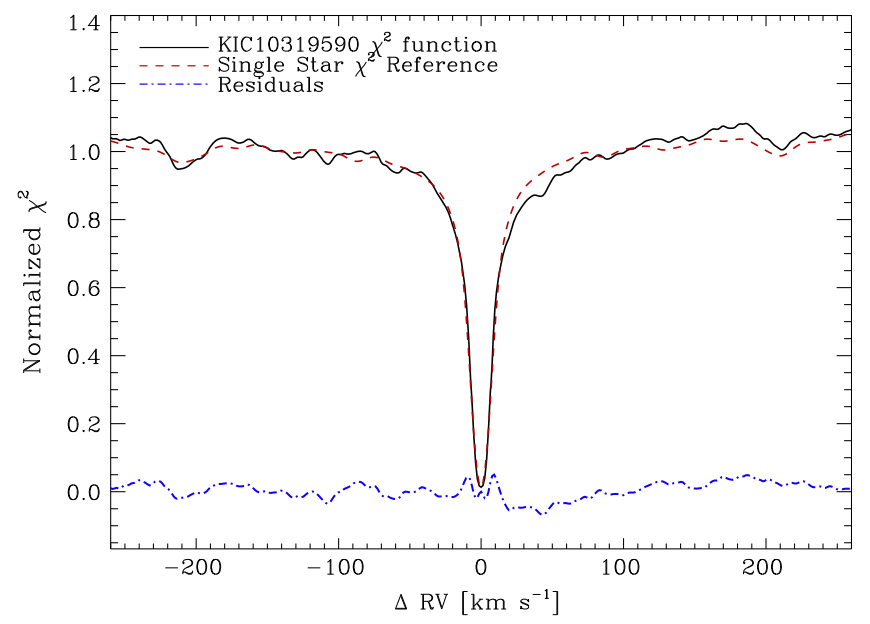

Figure 11. Primary star $\chi^{2}$ function for KIC 10319590. We can see a slight deviation from the characteristic single star $\chi^{2}$ function at $\Delta \mathrm{RV} \approx+45 \mathrm{~km} \mathrm{~s}^{-1}$, indicating a possible secondary star. This deviation alone, however, is not convincing enough to make any conclusive decisions.

\subsubsection{Secondary Star Detection Summarized}

Figure 9 has two panels, with the left showing the calibrated residual $\chi^{2}$ minimum as a function of $T_{\text {eff }}$ and the right showing $\chi^{2}$ versus $\Delta \mathrm{RV}$. This includes the minima curves for $1 \%$ and $3 \%$ injected secondaries, shown in red and blue, respectively (see Section 3.3.4).

This final Figure 9 is intended to summarize our knowledge about the secondary star: estimated $T_{\text {eff }}$, estimated percentage contribution to the total flux spectrum in the $\mathrm{V}$ and $\mathrm{R}$ bands, and the radial velocity of the secondary relative to the primary star. Any additional parameters for the secondary star cannot be determined accurately enough to be published.

On the final secondary star plot, we annotate any minimum that contributes at least $0.5 \%$ of the total flux. Depending on the $\mathrm{S} / \mathrm{N}$ of the original spectrum (typically 50-200 per pixel), the spectral types of the two stars, and their $\Delta R V$, secondaries with fluxes between $0.5 \%$ and $1 \%$ relative to the primary star can be detected with our algorithm.

We visually inspect each diagnostic plot to assess the probability that the identified minimum is indeed due to a secondary star. When there is no clear $\chi^{2}$ minimum, or when the $\chi^{2}$ minimum is due to fluctuations, we establish the threshold limits on the secondary star as follows. Any undetected secondary must contribute less to the total flux than would have been revealed above the fluctuations in the $\chi^{2}$ functions. If this percentage is not marked on the plot, the brightness limit is $0.5 \%$ relative to the primary star. Otherwise, the appropriate percentage is marked on the plot. M dwarf secondaries contributing more than $0.5 \%$ of the total flux can typically be detected in our spectra if present, or ruled out if not present.

\section{ASSESSING THE ALGORITHM: TESTS OF SYNTHETIC AND REAL BINARIES}

\subsection{Injection-recovery Experiment}

In order to test and calibrate our algorithm, we performed injection-recovery experiments. The goals were to estimate the uncertainty of the determined secondary star parameters, allow for the correction of any systematic errors, and identify the limitations of the algorithm.

We synthesized three sets of 360 binary stars, which were composed of pairs of our library spectra with added $2 \%$ Poisson noise. The noise level reflects that $(2 \%$ per pixel, i.e., $\mathrm{S} / \mathrm{N} \approx 45)$ common to many of the Keck-HIRES spectra we analyzed in this paper, namely the $1160 \mathrm{KOI}$, described in Section 5. Because the spectra used to synthesize the binaries are real observed spectra with Keck-HIRES, they already contain a certain amount of other types of noise (such as red noise), and thus the synthetic binaries accurately capture the nature of any other spectra analyzed in this paper.

We chose library stars in $T_{\text {eff }}$ increments of $500 \mathrm{~K}$, ranging from $3500 \mathrm{~K}$ to $6000 \mathrm{~K}$. We then created pairs of spectra for all possible permutations of $T_{\text {eff }}$, beginning at $3500 \mathrm{~K}$ for both the primary and the secondary star. We considered all
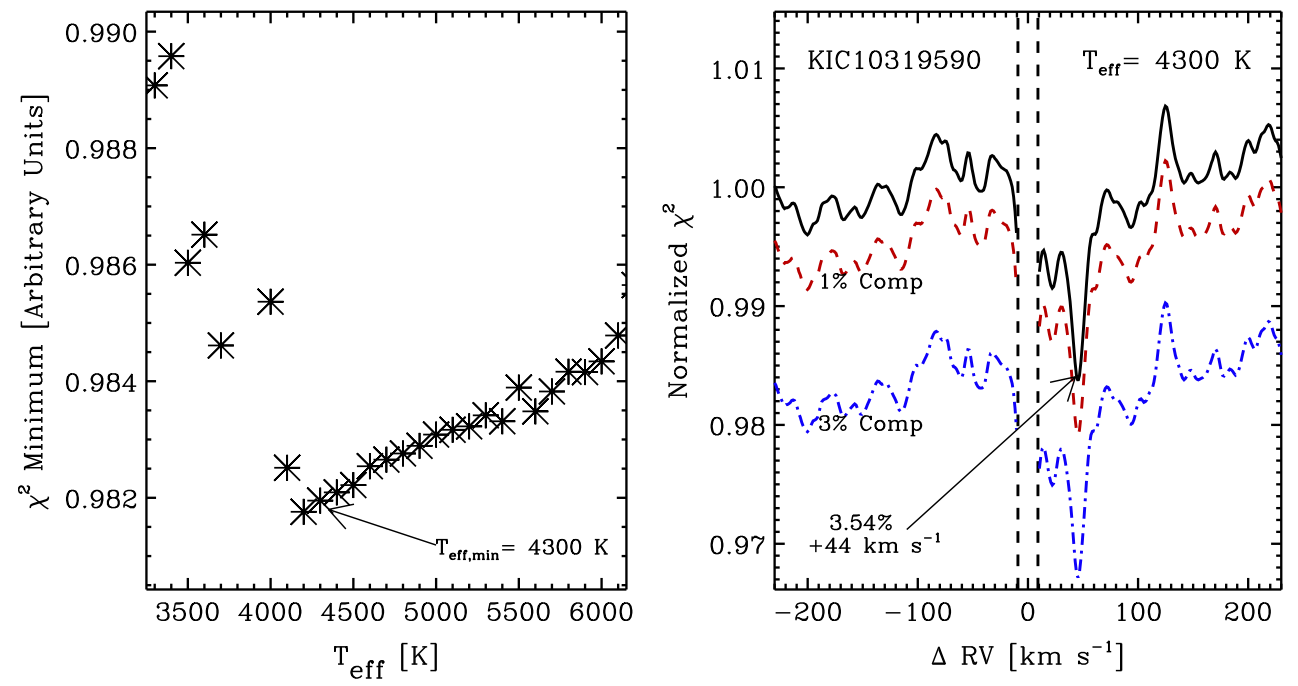

Figure 12. Final secondary star plot for KIC 103194950 . Same as Figure 9 . As foreshadowed by the Figure 11 , a secondary star is detected at $+44 \mathrm{~km} \mathrm{~s}{ }^{-1} \Delta \mathrm{RV}$, with an estimated $T_{\text {eff }}$ of $4300 \mathrm{~K}$ and contributing $3.54 \%$ to the total flux. 


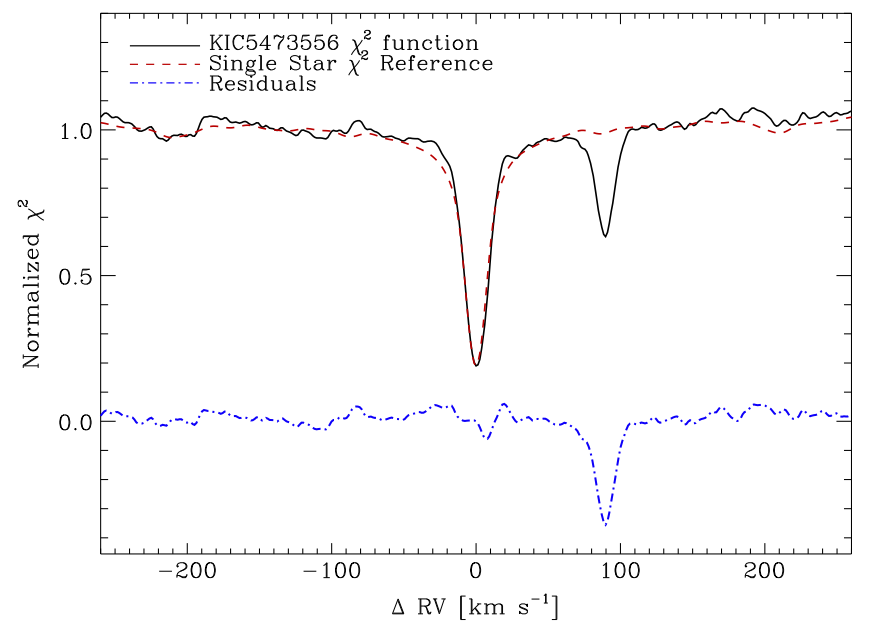

Figure 13. Primary star $\chi^{2}$ function for KIC 5473556. We can see a clear evidence of secondary star at $\Delta \mathrm{RV} \approx+90 \mathrm{~km} \mathrm{~s}^{-1}$. While this is a fairly convincing argument for the spectral binarity, residual $\chi^{2}$ function provides a greater insight into parameters for the secondary.

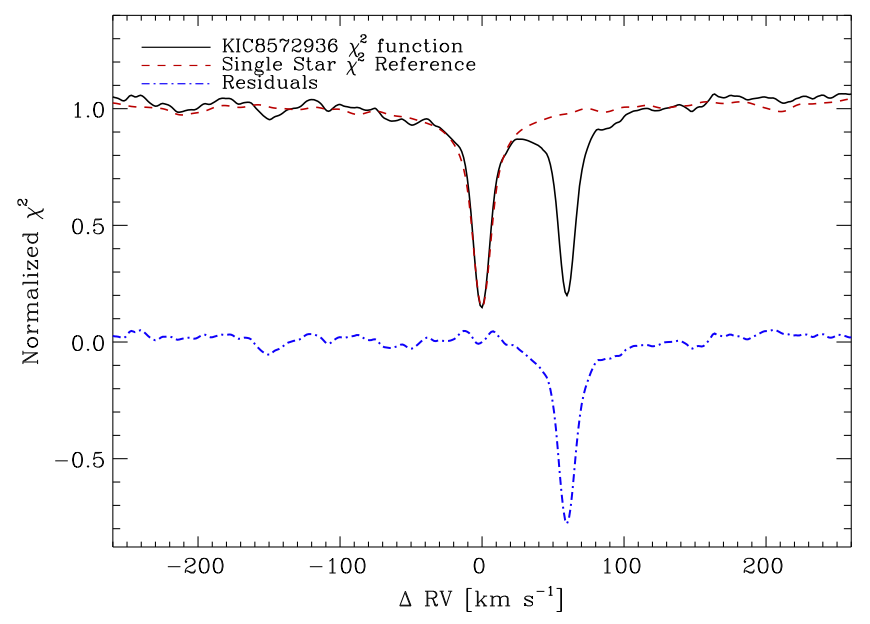

Figure 15. Primary star $\chi^{2}$ function for KIC 8572936. There is an almost equally bright secondary star detected at $\Delta \mathrm{RV} \approx+60 \mathrm{~km} \mathrm{~s}^{-1}$. Again, while this is a fairly convincing argument for the spectral binarity, a residual $\chi^{2}$ function provides a greater insight into parameters for the secondary star.
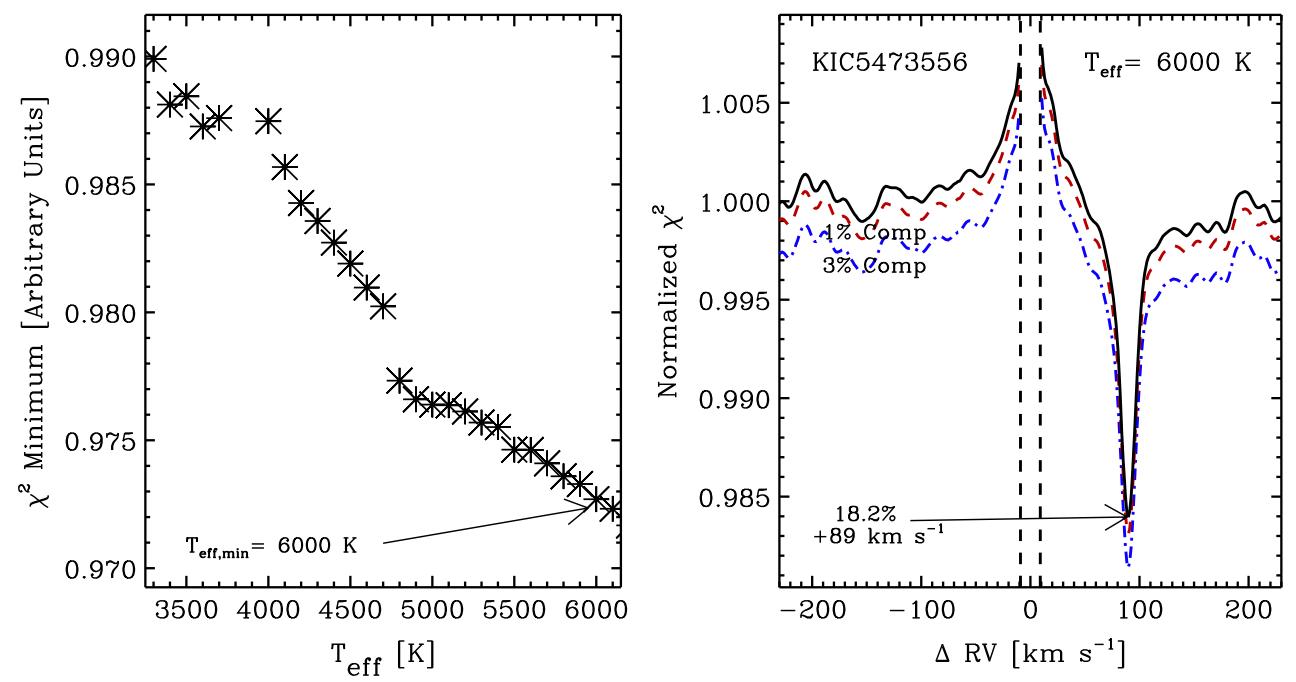

Figure 14. Final secondary star plot for KIC 5473556 . Same as Figure 9 . As indicated by the Figure 13 , a secondary star is detected at $+89 \mathrm{~km} \mathrm{~s} \mathrm{~s}^{-1} \Delta \mathrm{RV}$, with an estimated $T_{\text {eff }}$ of $6000 \mathrm{~K}$ and contributing $18.2 \%$ to the total flux.

possible combinations, including those where the primary star is cooler than the secondary, to account for both bound systems and background companions. The secondary set of absorption lines was shifted from the primary by $\Delta \mathrm{RV}$ of +50 $\mathrm{km} \mathrm{s}^{-1}$, where the two sets of lines are well separated. Tests have shown that for $\Delta R V$ separations larger than $\pm 10 \mathrm{~km} \mathrm{~s}^{-1}$ the detectability of the second star is independent of the relative radial velocity for the two stars. For smaller $\Delta \mathrm{RV}$ separations, the two sets of absorption lines overlap due to the natural broadening. At such small $\Delta \mathrm{RV}$ separations, we can only detect an M dwarf secondary to a G-type primary star. For those cases, additional tests have been carried out with the secondary set of absorption lines shifted from the primary by only $+5 \mathrm{~km} \mathrm{~s}^{-1}$.
Each of the three sets of 360 spectra differs by the relative brightness of the secondary star. We constructed cases in which the second star contributed $10 \%, 3 \%$, and $1 \%$ of the total flux in our optical spectra ( $\mathrm{V}$ and $\mathrm{R}$ bandpasses). For the cases of G-type primary and an M dwarf secondary star, we performed additional tests with even higher flux ratios, where the $\mathrm{M}$ dwarf secondary star contributed as low as $0.05 \%, 0.1 \%$ and $0.5 \%$ of the total flux. To obtain a statistically significant sample, we created 10 different binary spectra for each such permutation, each with a different realization of Poisson noise. We then blindly executed our search algorithm for secondary spectra on each synthetic binary. In the search, we removed the spectra used to construct that particular case from the library, preventing those from being chosen as best-fitting stars. 

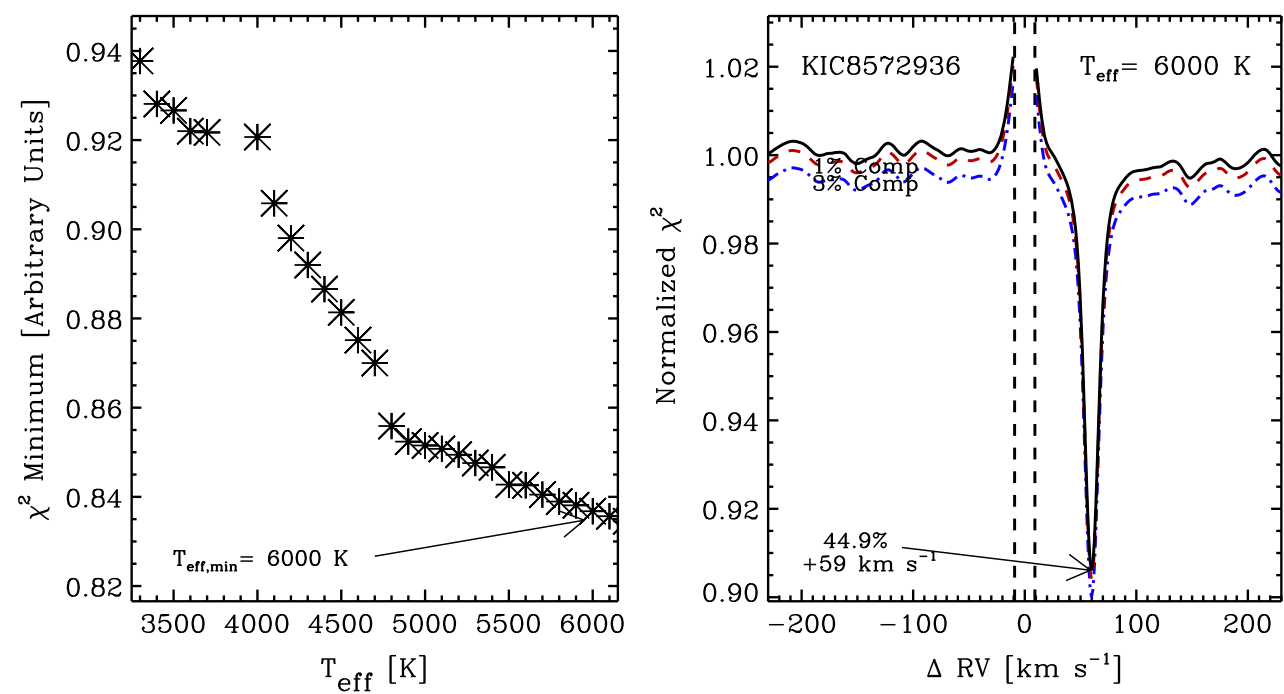

Figure 16. Final secondary star plot for KIC 8572936 . Same as Figure 9 . As already revealed by the Figure 13 , a secondary star is detected at $+59 \mathrm{~km} \mathrm{~s}{ }^{-1} \Delta \mathrm{RV}$, with an estimated $T_{\text {eff }}$ of $6000 \mathrm{~K}$ and contributing $44.9 \%$ to the total flux.

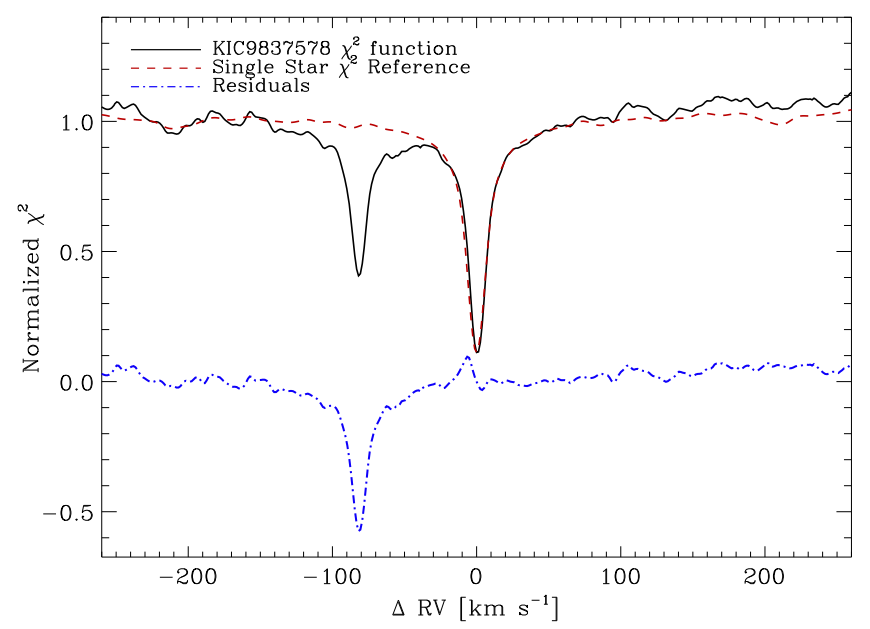

Figure 17. Primary star $\chi^{2}$ function for KIC 9837578 . A bright secondary star is detected at $\Delta \mathrm{RV} \approx-80 \mathrm{~km} \mathrm{~s}^{-1}$.

\subsubsection{Secondary Star Recovery Rate}

We used the synthetic binary spectra with added noise to determine the uncertainties in the deduced parameters for the secondary star. We investigated each temperature combination and each flux ratio separately. We examined the recovery rate by counting all the cases where the secondary star was identified, disregarding the accuracy of the estimated parameters. Results are summarized in Table 2. The table can be read as follows: $T_{\text {eff }}$ values running horizontally denote the effective temperature of the secondary star, and the values running vertically down the first column denote $T_{\text {eff }}$ of the primary star. For example, the $60 \%$ rate quoted in the last column of the first data row means that the secondary star in the binary spectrum consisting of $99 \%$ of a $3500 \mathrm{~K}$ star and $1 \% 3500 \mathrm{~K}$ star was successfully identified in 6 out of 10 cases.
For all synthetic spectra, we added a Poisson noise at the level of $2 \%$. Since we subtract the primary star absorption lines, a $1 \%$ secondary implies an $\mathrm{S} / \mathrm{N}$ for the residual spectrum of only $0.5,3 \%$ has an $\mathrm{S} / \mathrm{N}$ of 1.5 , and a $10 \%$ secondary yields a residual spectrum with an $\mathrm{S} / \mathrm{N}$ of 5 . Of course, such low $\mathrm{S} / \mathrm{N}$ values per pixel are overcome by the thousands of absorption lines, detected in thousands of pixels, each contributing to the detectability of the secondary star. Thus the effective $\mathrm{S} / \mathrm{N}$ of the secondary star is much higher than the per-pixel $\mathrm{S} / \mathrm{N}$.

\subsubsection{Calibration of the Secondary Star Temperature}

We isolated the synthetic binary cases where the binarity of the spectrum was established, and examined the predicted secondary star temperature as opposed to the actual values. As mentioned in Section 3.3.3, we noticed a dependence of the depth of the residual $\chi^{2}$ minimum on the temperature of the library spectrum, which was superimposed with the studied spectrum to construct the residual $\chi^{2}$. Except when the secondary star was an $\mathrm{M}$ dwarf, the residual $\chi^{2}$ minimum was always the deepest for $T_{\text {eff }} \sim 4500 \mathrm{~K}$, regardless of whether or not that was the actual $T_{\text {eff }}$ of the secondary star. We suspect that this bias is caused by the nature of the $4500 \mathrm{~K}$ spectrum itself, as it contains an abundance of deep and sharp absorption lines from neutral metals that dominate FGK spectra. With the primary star subtracted from the spectrum, the residuals are dominated by Poisson noise at a $2 \%$ level, especially when the secondary star contributes only a few percent to the total flux. These neutral metal absorption lines tend to accidentally align with the noise much more frequently due to their deep, sharp features. Such accidental alignments cause a deeper $\chi^{2}$ minimum for the $4500 \mathrm{~K}$ library star with the studied spectrum of any $T_{\text {eff }}$, particularly for the residuals with $\mathrm{S} / \mathrm{N}$ values near unity.

This bias was strong enough to influence the estimated temperature of the second star and make our deduced raw derived secondary star parameters inaccurate. In order to correct for this temperature bias, we calculated the average residual $\chi^{2}$ minimum distribution for all the test cases of a 

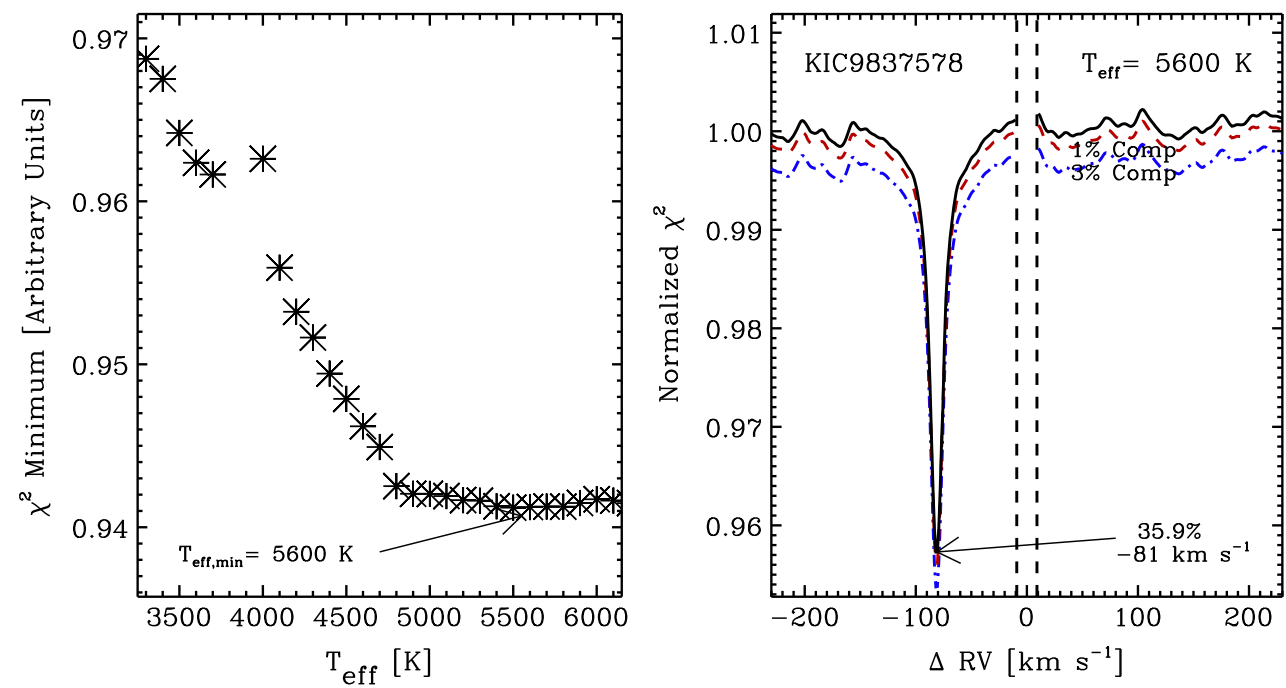

Figure 18. Final secondary star plot for KIC 9837578 . Same as Figure 9 . A secondary star is detected at $-81 \mathrm{~km} \mathrm{~s}^{-1} \Delta \mathrm{RV}$, with an estimated $T_{\text {eff }}$ of $5600 \pm 400 \mathrm{~K}$ and contributing $35.9 \%$ to the total flux.

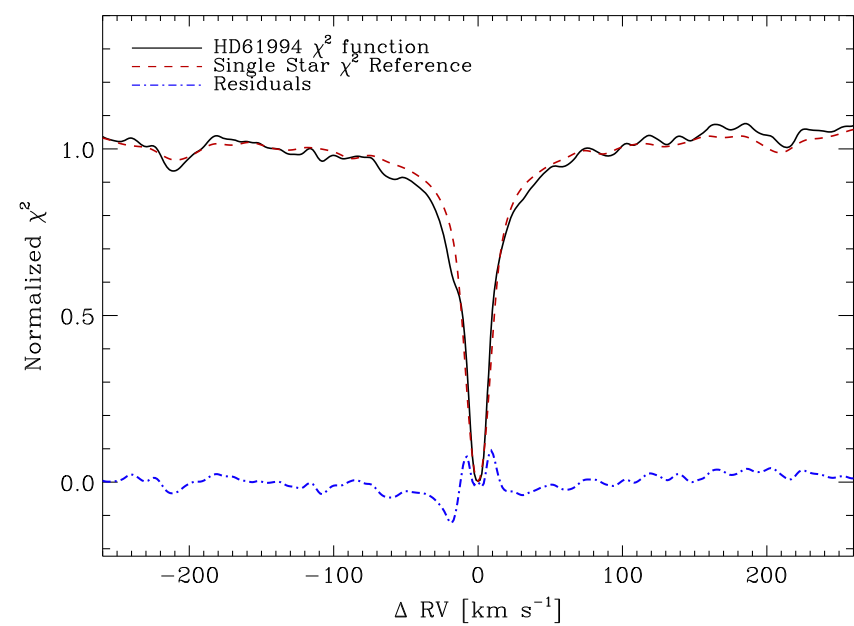

Figure 19. Primary star $\chi^{2}$ function for HD 61994. There is a slight asymmetry in the shape of the $\chi^{2}$ minimum, indicating a possible secondary star at $-20 \mathrm{~km} \mathrm{~s}^{-1}$. The asymmetry alone, however, is not conclusive evidence for the presence of another star, and we need to refer to the residual $\chi^{2}$ function for more details on a potential secondary.

particular flux ratio, excluding non-detections. If the distribution was unbiased against library spectrum's $T_{\text {eff }}$, the mean distribution should be approximately constant with respect to temperature, as we ensured that there were an equal number of cases at each secondary star $T_{\text {eff }}$.

We found, however, that this was not the case; the mean distribution peaked at $4500 \mathrm{~K}$, as expected. Since the shape of any true $\chi^{2}$ minimum distribution will be affected by this peak, we use the mean distribution, normalized such that the maximum occurs at $\chi^{2}=1$, as our calibration function. Calibration functions for a $1 \%, 3 \%$, and $10 \%$ secondary star are shown in Figure 10.

As we can see from Figure 10, calibration functions are almost identical among the different relative brightnesses of the

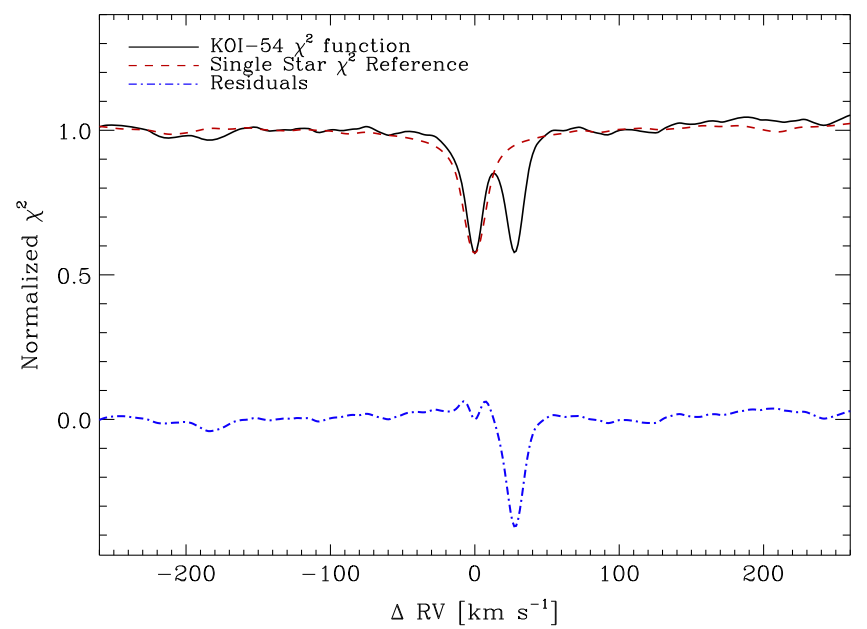

Figure 20. Primary star $\chi^{2}$ function for KOI-54. $\chi^{2}$ minimum is shallow compared to other examples because the NSO Stellar spectrum used to construct the $\chi^{2}$ functions differs significantly from the spectrum of a $8800 \mathrm{~K}$ star. The companion detected at $\approx+30 \mathrm{~km} \mathrm{~s}^{-1}$ appears equally bright.

two stars. Therefore, there will be negligible errors resulting from dividing a residual $\chi^{2}$ minimum distribution by these calibration functions for cases where the relative brightness of the studied star is not $1 \%, 3 \%$, or $10 \%$ exactly.

\subsubsection{Secondary Star Parameters}

Using calibrated $\chi^{2}$ minimum distribution, we estimated the second star temperature and relative brightness, as described in Sections 3.3.3 and 3.3.4. We then compared the deduced values to the known parameters, and the discrepancies are shown in Tables 3 and 4, respectively.

Table 3 lists the discrepancies in the SpecMatch $T_{\text {eff }}$ value and that derived from our algorithm, $\left(T_{\text {SpecMatch }}-T_{\text {deduced }}.\right)$ Table 4 lists the discrepancies in the set secondary star 

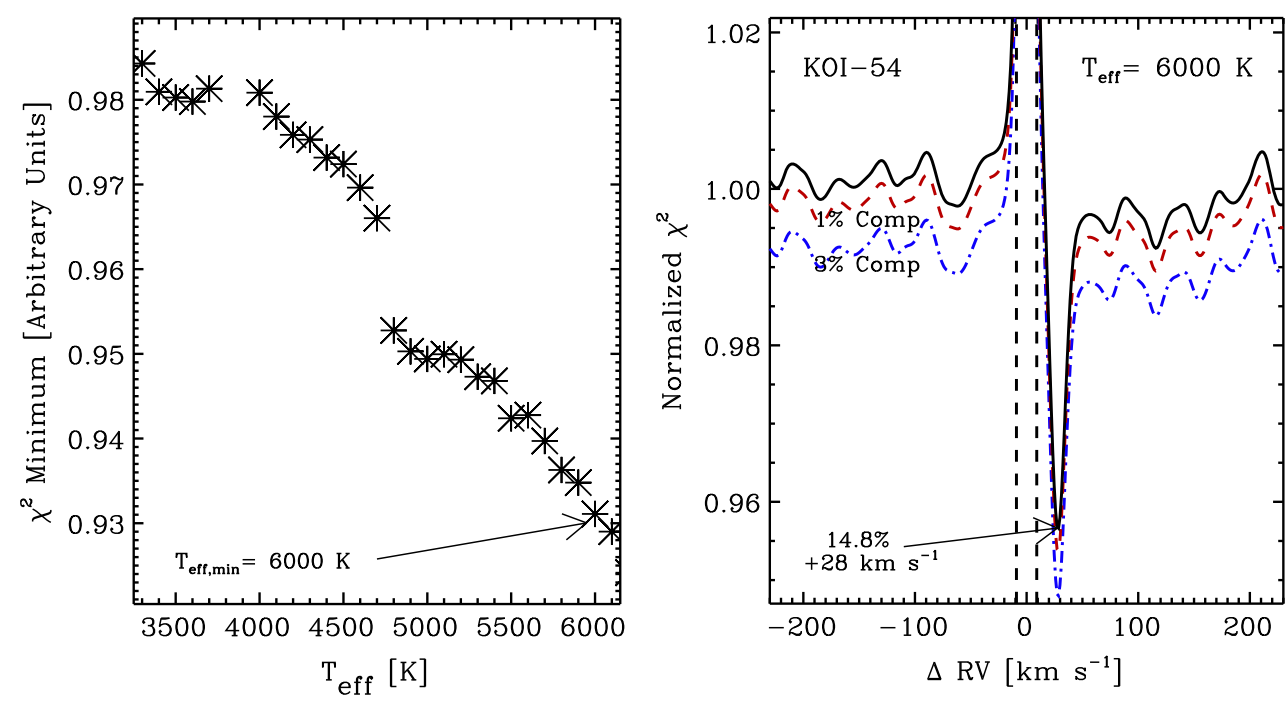

Figure 21. Final secondary star plot for KOI-54. Same as Figure 9. A secondary star is detected at $+28 \mathrm{~km} \mathrm{~s}^{-1} \Delta \mathrm{RV}$, with an estimated $T_{\text {eff }}$ of $6200 \mathrm{~K}$ and contributing $17.4 \%$ to the total flux. This low relative brightness is surprising, as the two minima appeared almost equal in Figure 20. For this system, however, both constituent stars are $\sim 2000 \mathrm{~K}$ hotter than the hottest star in the SpecMatch library, thus our derived parameters are highly inaccurate.

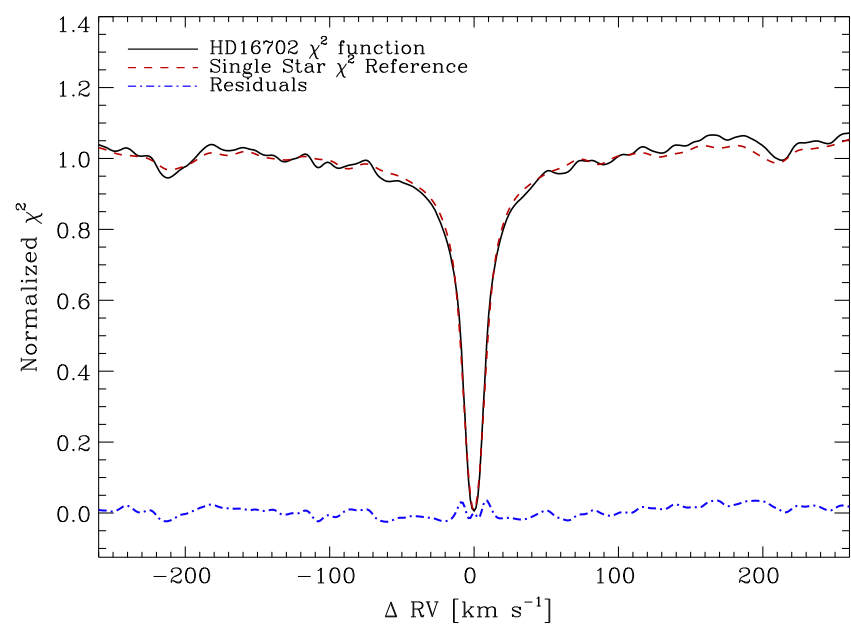

Figure 22. Primary star $\chi^{2}$ function for HD 16702. Because the companion is very faint and separated only by $5 \mathrm{~km} \mathrm{~s}^{-1}$ from the primary star, the primary $\chi^{2}$ function alone shows no evidence of spectrum binarity.

percentage contribution to the total flux and the value derived by our algorithm. We know the actual flux contribution precisely, because we artificially scaled down the secondary spectra when constructing synthetic binaries. The values are listed in terms of the percentage of the total flux, $\left(\%_{\text {known }}-\%_{\text {deduced }}\right)$. Each primary-secondary temperature combination consisted of 10 trial cases, with different realizations of added Poisson noise.

We report both the systematic difference (the mean discrepancy) and the associated standard deviation. Since the systematic differences are not constant over all temperature combinations or relative brightnesses, we cannot apply a single correction to obtain more accurate values. As such, Tables 3 and 4 serve more as illustrations of the injection-recovery results.
We noticed larger $T_{\text {eff }}$ systematic differences for $4000 \mathrm{~K}$ secondary stars. This is mostly due to the scarce SpecMatch library populations in that region, with some $100 \mathrm{~K} T_{\text {eff }}$ intervals missing altogether. On the other hand, the algorithm did consistently correctly identify the $\mathrm{M}$ dwarf secondaries, owing their detectability to their distinct spectral features. As expected, the systematic differences in $T_{\text {eff }}$ and the standard deviations decreased for brighter secondary stars, as the secondary spectrum becomes brighter than the $2 \%$ noise.

On the other hand, absolute errors in percentage increased when the secondary star was brighter. This was expected because the errors in the linear extrapolations of $\chi^{2}$ minimum depth become larger for brighter stars. Nevertheless, the errors were still mostly only around $1 \%$, and the relative errors rarely exceed $50 \%$. We also noticed a pattern that the relative brightness of hotter secondary stars was mostly overestimated, while cooler stars were underestimated. These trends can be useful when establishing limits on brightnesses of any real secondaries.

We combine the systematic error and the uncertainty to form more conservative uncertainties to be used with the secondary star parameters deduced in the analysis of actual spectra. These are shown in Table 5. Columns two through seven show the absolute uncertainty in the effective temperature of the secondary star for several possible primary-secondary star pairs; columns eight through 13 show the relative uncertainty in the flux ratio. Based on the effective temperatures of the primary-secondary pair and their flux ratio, appropriate uncertainty for both the effective temperature of the secondary star and its relative brightness can be read from Table 5 .

\subsubsection{G-type Primary and $M d$ warf Secondary Star}

As a part of the injection-recovery experiment, we also more closely examined the case of a G-type primary star with a temperature of $5500 \mathrm{~K}$ and an M dwarf secondary at $3500 \mathrm{~K}$. Due to its low temperature, the $\mathrm{M}$ dwarf spectrum contains many molecular lines in the visible spectrum, which isquite 

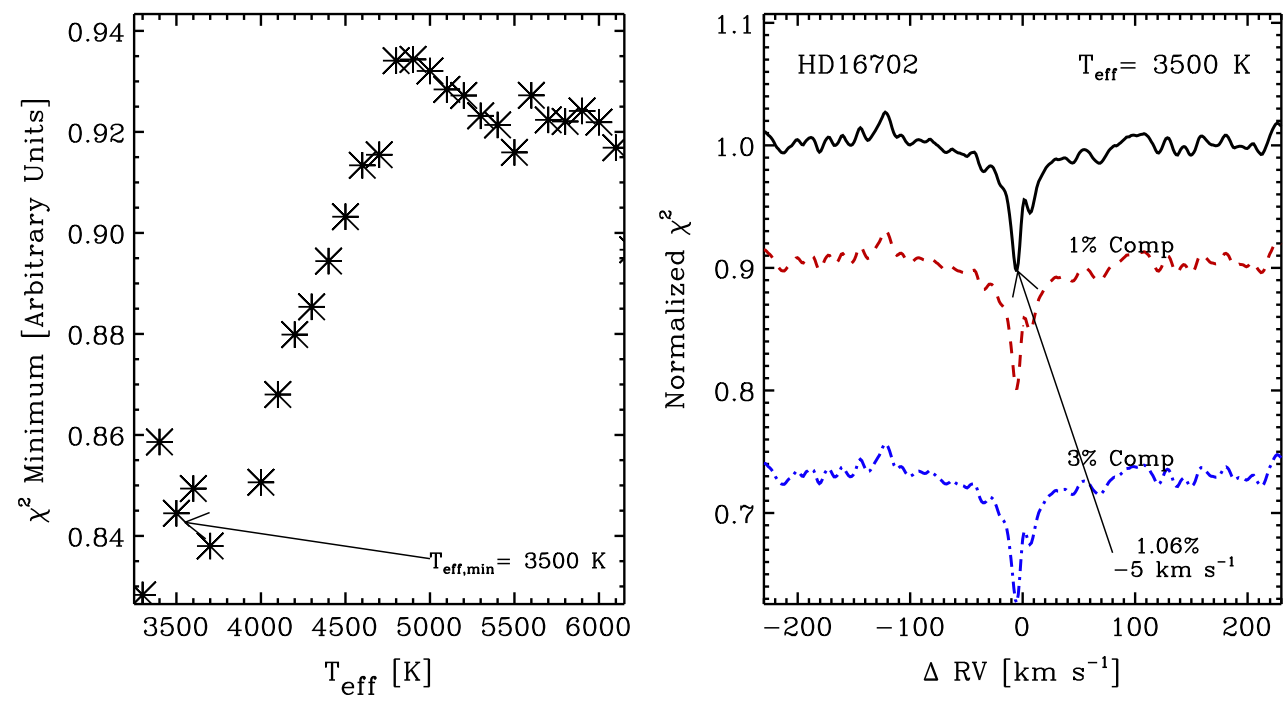

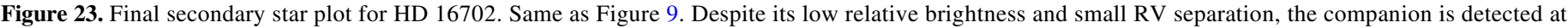
$\Delta \mathrm{RV}=-5 \mathrm{~km} \mathrm{~s}^{-1}$. The estimated companion temperature is $3500 \pm 250 \mathrm{~K}$ and it contributes $1.06 \%$ of the total flux.

distinct from the spectrum of FGK-type stars. As such, it is much easier to detect an $\mathrm{M}$ dwarf secondary spectra, even when the $\Delta \mathrm{RV}$ for the two stars is low.

We considered a situation where the two stars are separated by only $5 \mathrm{~km} \mathrm{~s}^{-1}$, placing the secondary set of absorption lines into the masked-out region on our residual $\chi^{2}$ versus $\Delta R V$ plot. We constructed three sets of 20 different synthetic binary stars with a $5500 \mathrm{~K}$ primary and a $3500 \mathrm{~K}$ secondary star, with the secondary contributing either $1 \%, 3 \%$, or $5 \%$ of the total flux. We then executed the algorithm on each spectrum, determined the recovery rate, and calculated the discrepancies in the deduced $T_{\text {eff }}$ and percentage flux. Results are shown in Table 6. Each companion percentage contribution sample consisted of 20 test cases.

These results quantify the ability of our algorithm to detect M dwarf secondary stars even when the two sets of absorption lines overlap. Some of the M dwarf absorption lines still get subtracted away together with the primary star, thus the relative brightness of the secondary was consistently underestimated for all cases. This suggests an important limitation on our algorithm; when the secondary stars are detected in the $\Delta \mathrm{RV}<$ $10 \mathrm{~km} \mathrm{~s}^{-1}$ region, the estimated relative brightness of the second star is more of a lower limit rather than an actual value.

Besides considering $\mathrm{M}$ dwarf secondary stars with low $\Delta \mathrm{RV}$ separation, we also explored the detectability limit in terms of the relative brightness of the two stars. We constructed synthetic binaries where the $\mathrm{M}$ dwarf secondary contributed $0.05 \%, 0.1 \%$, and $0.5 \%$ of the total flux. The recovery rate for the $0.5 \% \mathrm{M}$ dwarf secondary star was $40 \%$, whereas none of the fainter companions were detected. Since all of the spectra had a $2 \%$ percent noise, this places the M dwarf detectability to an $\mathrm{S} / \mathrm{N} \sim 0.25$.

\subsection{Known Real Spectroscopic Binaries}

We executed our algorithm on several cases of known spectroscopic binaries. We then compared our results to those available in the literature. Results are summarized in Table 7 for the primary star parameters, and in Table 8 for the secondary. The uncertainties for the secondary star parameter are based on the Table 5. Even though we normally do not report the parameters of the primary star, we include them in this experiment. This is to demonstrate that even though our primary star parameters might carry larger errors and uncertainties than those derived using alternative methods, they still pose a sufficient accuracy for the purposes of the subtraction of the primary star absorption lines.

\subsubsection{KIC 10319590}

KIC 10319590 is a precessing eclipsing binary, with timevarying eclipse depths (Slawson 2011; Rappaport et al. 2013). There is very little evidence of the secondary star in the $\chi^{2}$ function for the primary star shown in Figure 11, indicating that the secondary is faint.

The residual $\chi^{2}$ function shown in Figure 12 shows evidence of a secondary set of absorption lines at $\Delta \mathrm{RV}=+44 \mathrm{~km} \mathrm{~s}^{-1}$. Based on the residual $\chi^{2}$ minimum distribution, we estimate the secondary star $T_{\text {eff }}=4300 \pm 500 \mathrm{~K}$, with uncertainty based on Table 5.

Deduced percentage of the total flux for the secondary star is $3.54 \%$, yielding the flux ratio $\mathrm{F}_{B} / \mathrm{F}_{A}=0.036 \pm 0.07$. The comparison of our results to known parameters is limited by the lack of the available information.

\subsubsection{KIC 5473556}

KIC 5473556 is another example of a SB2 eclipsing binary (R. Angus 2013, private communication). For this system, the companion is sufficiently bright to cause a secondary minimum in the $\chi^{2}$ function for the primary star, as shown in Figure 13.

There is a clear detection shown in the residual $\chi^{2}$ (Figure 14) at $\Delta \mathrm{RV}=+89 \mathrm{~km} \mathrm{~s}^{-1}$. We estimate the secondary star $T_{\text {eff }}$ at $6000 \pm 100 \mathrm{~K}$. At the relative flux of $\mathrm{F}_{B} / \mathrm{F}_{A}=0.22$ \pm 0.07 , this could either be a bound companion or a background star, as the primary star $T_{\text {eff }}$ is similar to the estimated secondary star value. 
In order to resolve the ambiguity, we executed our algorithm using a different observation taken at a different time. If the two stars orbit each other, a different orbital phase should yield a different $\Delta \mathrm{RV}$ value. This was indeed the case, as another observation shows a companion at $\Delta \mathrm{RV} \approx-115$ $\mathrm{km} \mathrm{s}^{-1}$.

The flux ratio of $0.22 \pm 0.07$ and approximately the same $T_{\text {eff }}$ for the two stars indicates that our deduced companion temperature might be an overestimate, and the actual companion $T_{\text {eff }}$ most likely lies at the lower uncertainty limit, $T_{\text {eff }} \sim 5900 \mathrm{~K}$. Moreover, it is possible that only a fraction of the flux from the companion actually entered the slit during the observation. Since the parameters for the companion of KIC 5473556 are not known, we cannot compare our derived flux ratio, $\mathrm{F}_{B} / \mathrm{F}_{A}=0.22 \pm 0.07$, to a known value.

\subsubsection{KIC 8572936}

KIC 8572936 is an SB2 binary system that is also known for its circumbinary planet, Kepler-34 (Welsh et al. 2012). The two stars are both Sun-like stars, and their fluxes are comparable; $\mathrm{F}_{B} / \mathrm{F}_{A}=0.8475 \pm 0.005$ (Welsh et al. 2012). The effective temperatures of the two stars are similar, $T_{\text {eff,A }}$ $=5932 \pm 130 \mathrm{~K}$ and $T_{\text {eff }, \mathrm{B}}=5867 \pm 130 \mathrm{~K}$.

This is an interesting case due to the similarity between the two stars, both in their spectral types and in the fractional contribution to the total flux. The main problem with such cases is that the two sets of absorption lines interfere with the best-fit for the primary star, thus we inevitably subtract some of the secondary lines together with the primary star. This leads to a somewhat larger uncertainty in the derived flux ratio for the stars using our method; however, the detection of both stars is clear and unambiguous, as seen in Figures 15 and 16.

The estimated temperature of the companion of $T_{\text {eff }}=6000$ $\pm 100 \mathrm{~K}$, is in agreement with $T_{\text {eff }}=5812 \pm 150 \mathrm{~K}$ quoted by Welsh et al. (2012). We calculated the flux ratio of $\mathrm{F}_{B} / \mathrm{F}_{A}$ $=0.82 \pm 0.25$, which, in the uncertainty limit, also matches the Welsh et al. (2012) value of $0.8475 \pm 0.005$.

\subsubsection{KIC 9837578}

The case of KIC 9837578 is similar to KIC 8572936. It is also an SB2 binary system, with the two stars of similar $T_{\text {eff }}$, but the flux ratio in the Kepler bandpass is somewhat lower, $\mathrm{F}_{B} / \mathrm{F}_{A}$ $=0.3941$ (Welsh et al. 2012).

We find a secondary star at $\Delta \mathrm{RV}=-81 \mathrm{~km} \mathrm{~s}^{-1}$, with the estimated effective temperature of $5600 \pm 400 \mathrm{~K}$ (Figures 17 and 18). Taking into account the uncertainty, $T_{\text {eff }}$ agrees with the Welsh et al. (2012) value of $5202 \pm 100 \mathrm{~K}$. The estimated flux ratio for the two stars of $0.56 \pm 0.14$ is larger than the Welsh et al. (2012) value by $7 \%$. Some of this discrepancy can be attributed to a slightly different wavelength bandpass of our spectra as compared to Welsh et al. (2012), and some due to the mixing of the two sets of absorption lines, as both stars are of relatively similar spectral types with the secondary star contributing a significant amount of the total flux.

\subsubsection{HD 61994}

HD 61994 is a double-lined spectroscopic binary with a period of 552.8 days (Strassmeier et al. 2012). When the spectrum was taken, the relative radial velocity for the two stars was only $+18 \mathrm{~km} \mathrm{~s}^{-1}$, which is an interesting example for cases where the relative radial velocity is low and the secondary star is something other than an $M$ dwarf. Furthermore, the companion is faint - the visible brightness ratio for the two stars $\mathrm{F}_{B} / \mathrm{F}_{A}$ is only 0.069 (Strassmeier et al. 2012).

The primary $\chi^{2}$ deviates slightly from the characteristic single-star $\chi^{2}$ function at $\Delta \mathrm{RV} \approx-20 \mathrm{~km} \mathrm{~s}^{-1}$, shown in Figure 19. However, more convincing evidence for the presence of a secondary star comes from the residual $\chi^{2}$ function shown in Figure 9, indicating a secondary star at $\Delta \mathrm{RV}=-16 \mathrm{~km} \mathrm{~s}^{-1}$. Its residual $\chi^{2}$ minimum distribution function sets the secondary star $T_{\text {eff }}$ at $4200 \pm 650 \mathrm{~K}$. Within the uncertainty limit, this agrees with the Strassmeier et al. (2012) value of $T_{\text {eff }}=4775 \pm 150 \mathrm{~K}$. The calculated flux ratio, $\mathrm{F}_{B} / \mathrm{F}_{A}=0.055 \pm 0.022$, also matches the flux ratio of $\mathrm{F}_{B} / \mathrm{F}_{A}=0.069$, quoted by Strassmeier et al. (2012).

\subsection{6. $\mathrm{KOI}-54$}

KOI-54 is a non-eclipsing, SB2 binary system. The two stars are in an almost face-on, highly eccentric orbit (Welsh et al. 2011).

This example shows the ability to detect the presence of both stars even when their effective temperatures are outside the range of our library and above the highest SpecMatch library spectrum temperature used to construct the residual $\chi^{2}$ panels. The primary star has a temperature $T_{\text {eff }}=8800 \pm 200 \mathrm{~K}$, and the companion lags behind this value only slightly with $T_{\text {eff }}$ $=8500 \pm 200 \mathrm{~K}$ (Welsh et al. 2011). This is over $2000 \mathrm{~K}$ higher than any of our library spectra.

Nonetheless, we are able to see the minimum in the $\chi^{2}$ function shown in Figure 20 due to each of the two stars, as well as detect a clear minimum indicating the presence of the companion after the primary star was subtracted, as seen in Figure 21. We do not state the parameters obtained for KOI-54 system, because they are incorrect due to the large discrepancy between the stellar spectra for the KOI-54 stars and the available library spectra; more on this issue is explained in Section 6.2.

\subsection{7. $H D 16702$}

HD 16702 is identified as a binary star in Díaz et al. (2012), with the mass ratio for the companion and the primary star being $\mathrm{M}_{B} / \mathrm{M}_{A}=0.35-0.41$ (Díaz et al. 2012). We detect a secondary star at $\Delta \mathrm{RV}=-5 \mathrm{~km} \mathrm{~s}^{-1}$, with an estimated $T_{\text {eff }}$ $=3500 \pm 250 \mathrm{~K}$ and contributing $1.06 \%$ of the total flux, as shown in Figures 22 and 23.

Since the only known companion parameter is its mass, we performed some analysis to confirm that our detection agrees with Díaz et al. (2012) value of $0.40 \pm 0.05 M_{\odot}$. The spectrum was taken when the velocity of both stars was almost exclusively along the line of sight, so we can predict the mass of the companion based on their radial velocity separation. Using nine precise RV measurements (Marcy \& Butler 1992), we determine that the primary star velocity relative to the center of mass of the binary system is at its maximum at the time of observation, $v_{p}=-2.2 \mathrm{~km} \mathrm{~s}^{-1}$. Using the primary mass of 0.98 $M_{\odot}$ (Díaz et al. 2012), and $\Delta \mathrm{RV}$ of $-5 \mathrm{~km} \mathrm{~s}^{-1}$, as determined by our algorithm, we predict the mass ratio $\mathrm{M}_{B} / \mathrm{M}_{A}=0.30$. This indicates a companion mass of $\sim 0.3 \mathrm{~km} \mathrm{~s}^{-1}$, which is only slightly below the Díaz et al. (2012) value. Using known calibrations, both the flux ratio of $\sim 0.01$ and the companion 
Table 9

California Kepler Search: Binary Systems

\begin{tabular}{|c|c|c|c|c|c|}
\hline \multirow[b]{2}{*}{ KOI } & \multirow{2}{*}{$\begin{array}{c}\text { Primary Star } \\
T_{\text {eff,A }}(\mathrm{K}) \\
\end{array}$} & \multicolumn{3}{|c|}{ Companion Parameters } & \multirow{2}{*}{$\begin{array}{c}\text { Planetary } \\
\text { Data }^{a}\end{array}$} \\
\hline & & $T_{\text {eff,B }}(\mathrm{K})$ & $\mathrm{F}_{B} / \mathrm{F}_{A}$ & $\Delta \mathrm{RV}\left(\mathrm{km} \mathrm{s}^{-1}\right)$ & \\
\hline 5 & $5753 \pm 75$ & $5900 \pm 850$ & $\geqslant 0.066 \pm 0.020$ & +11 & $2 \mathrm{PC}$ \\
\hline 151 & $6276 \pm 163$ & $3500 \pm 250$ & $\geqslant 0.012 \pm 0.006$ & +14 & $1 \mathrm{PC}$ \\
\hline 219 & $5513 \pm 184$ & $\geqslant 6000 \pm 100$ & $\geqslant 0.330 \pm 0.033$ & +13 & $1 \mathrm{PC}$ \\
\hline 652 & $4700 \pm 128$ & $3700 \pm 150$ & $0.092 \pm 0.028$ & +22 & $1 \mathrm{PC}$ \\
\hline$\cdots$ & $\ldots$ & $4000 \pm 350$ & $0.020 \pm 0.007$ & +46 & $\ldots$ \\
\hline$\cdots$ & $\ldots$ & $3500 \pm 150$ & $0.006 \pm 0.002$ & -44 & $\ldots$ \\
\hline 698 & $6120 \pm 196$ & $4800 \pm 600$ & $0.048 \pm 0.005$ & +29 & $1 \mathrm{PC}$ \\
\hline 969 & $6224 \pm 186$ & $\geqslant 6000 \pm 100$ & $0.821 \pm 0.246$ & -42 & $1 \mathrm{PC}$ \\
\hline 1020 & $6058 \pm 158$ & $6000 \pm 100$ & $0.292 \pm 0.088$ & +39 & $1 \mathrm{PC}$ \\
\hline 1121 & $5671 \pm 156$ & $6000 \pm 100$ & $\geqslant 0.077 \pm 0.008$ & -12 & $1 \mathrm{PC}$ \\
\hline 1137 & $5324 \pm 178$ & $3600 \pm 150$ & $\geqslant 0.020 \pm 0.004$ & +11 & $1 \mathrm{PC}$ \\
\hline 1152 & $3806 \pm 50$ & $4200 \pm 350$ & $0.307 \pm 0.138$ & +27 & $1 \mathrm{FP}$ \\
\hline 1227 & $5658 \pm 159$ & $\geqslant 6000 \pm 100$ & $0.821 \pm 0.082$ & -123 & $1 \mathrm{PC}$ \\
\hline 1326 & $5378 \pm 173$ & $\geqslant 6000 \pm 100$ & $0.395 \pm 0.040$ & +42 & $1 \mathrm{PC}$ \\
\hline 1361 & $4014 \pm 76$ & $3600 \pm 200$ & $0.022 \pm 0.007$ & +40 & $1 \mathrm{CP}$ \\
\hline 1452 & $7172 \pm 211$ & $\cdots$ & $\cdots$ & +81 & $1 \mathrm{PC}$ \\
\hline 1613 & $6044 \pm 117$ & $\geqslant 6000 \pm 850$ & $\geqslant 0.044 \pm 0.013$ & +10 & $2 \mathrm{PC}$ \\
\hline 1645 & $5193 \pm 170$ & $4500 \pm 200$ & $0.287 \pm 0.029$ & +55 & $1 \mathrm{PC}$ \\
\hline 1684 & $6428 \pm 134$ & $\geqslant 6000 \pm 100$ & $0.300 \pm 0.090$ & -50 & $1 \mathrm{PC}$ \\
\hline 1784 & $5936 \pm 150$ & $\geqslant 6000 \pm 100$ & $\geqslant 0.192 \pm 0.058$ & -13 & $1 \mathrm{PC}$ \\
\hline 1796 & $6065 \pm 205$ & $3500 \pm 250$ & $0.012 \pm 0.006$ & +36 & $1 \mathrm{PC}$ \\
\hline 2059 & $4996 \pm 103$ & $3600 \pm 250$ & $0.016 \pm 0.008$ & +5 & $2 \mathrm{PC}$ \\
\hline 2075 & $6403 \pm 159$ & $3400 \pm 250$ & $\geqslant 0.008 \pm 0.004$ & -13 & $1 \mathrm{PC}$ \\
\hline 2215 & $5974 \pm 187$ & $\geqslant 6000 \pm 100$ & $0.185 \pm 0.056$ & +32 & $1 \mathrm{PC}$ \\
\hline$\ldots$ & $\ldots$ & $\geqslant 6000 \pm 100$ & $\geqslant 0.155 \pm 0.047$ & -15 & $\ldots$ \\
\hline 2311 & $5754 \pm 147$ & $5600 \pm 400$ & $\geqslant 0.276 \pm 0.069$ & +11 & $3 \mathrm{PC}$ \\
\hline 2457 & $6728 \pm 155$ & $\geqslant 6000 \pm 100$ & $\geqslant 0.076 \pm 0.023$ & +14 & $1 \mathrm{PC}$ \\
\hline 2787 & $6335 \pm 177$ & $\geqslant 6000 \pm 100$ & $0.222 \pm 0.067$ & +16 & $1 \mathrm{FP}$ \\
\hline 2813 & $5143 \pm 164$ & $\geqslant 6000 \pm 100$ & $0.195 \pm 0.058$ & +26 & $1 \mathrm{PC}$ \\
\hline 2867 & $4865 \pm 194$ & $4700 \pm 200$ & $0.389 \pm 0.136$ & +44 & $1 \mathrm{PC}$ \\
\hline 2965 & $6468 \pm 159$ & $\geqslant 6000 \pm 850$ & $0.022 \pm 0.007$ & +20 & $1 \mathrm{FP}$ \\
\hline 3000 & $5919 \pm 157$ & $4100 \pm 450$ & $\geqslant 0.055 \pm 0.019$ & +10 & $1 \mathrm{FP}$ \\
\hline 3002 & $6060 \pm 152$ & $\geqslant 6000 \pm 100$ & $0.475 \pm 0.143$ & -15 & $1 \mathrm{FP}$ \\
\hline 3035 & $6324 \pm 174$ & $\geqslant 6000 \pm 100$ & $\geqslant 0.212 \pm 0.064$ & +18 & $1 \mathrm{FP}$ \\
\hline 3161 & $6790 \pm 195$ & $\geqslant 6000 \pm 100$ & $0.305 \pm 0.092$ & -167 & $1 \mathrm{PC}$ \\
\hline 3162 & $6109 \pm 165$ & $5900 \pm 100$ & $\geqslant 0.553 \pm 0.166$ & -13 & $1 \mathrm{FP}$ \\
\hline 3216 & $5615 \pm 166$ & $\geqslant 6000 \pm 100$ & $\geqslant 0.326 \pm 0.033$ & +13 & $1 \mathrm{FP}$ \\
\hline 3231 & $6195 \pm 188$ & $\geqslant 6000 \pm 100$ & $0.175 \pm 0.053$ & -22 & $1 \mathrm{FP}$ \\
\hline 3243 & $6348 \pm 146$ & $\geqslant 6000 \pm 100$ & $0.590 \pm 0.177$ & +45 & $1 \mathrm{FP}$ \\
\hline 3415 & $5619 \pm 178$ & $5600 \pm 200$ & $0.786 \pm 0.157$ & -71 & $1 \mathrm{PC}$ \\
\hline 3471 & $4847 \pm 118$ & $4600 \pm 200$ & $0.746 \pm 0.079$ & -33 & $1 \mathrm{FP}$ \\
\hline$\ldots$ & $\ldots$ & $\geqslant 6000 \pm 100$ & $0.202 \pm 0.061$ & +26 & $\ldots$ \\
\hline$\ldots$ & $\ldots$ & $4100 \pm 300$ & $0.117 \pm 0.041$ & -52 & $\ldots$ \\
\hline 3506 & $6878 \pm 194$ & $\geqslant 6000 \pm 100$ & $0.206 \pm 0.062$ & +137 & $1 \mathrm{PC}$ \\
\hline 3515 & $6395 \pm 155$ & $\geqslant 6000 \pm 100$ & $0.134 \pm 0.040$ & +48 & $1 \mathrm{FP}$ \\
\hline 3527 & $5668 \pm 153$ & $\geqslant 6000 \pm 100$ & $0.880 \pm 0.264$ & -16 & $1 \mathrm{PC}$ \\
\hline 3528 & $6061 \pm 200$ & $\geqslant 6000 \pm 100$ & $0.182 \pm 0.055$ & +66 & $1 \mathrm{PC}$ \\
\hline 3557 & $6462 \pm 161$ & $\geqslant 6000 \pm 100$ & $0.393 \pm 0.118$ & +55 & $1 \mathrm{FP}$ \\
\hline 3573 & $6403 \pm 158$ & $\geqslant 6000 \pm 100$ & $\geqslant 0.109 \pm 0.033$ & -15 & $1 \mathrm{PC}$ \\
\hline 3583 & $7591 \pm 230$ & $\geqslant 6000 \pm 100$ & $\geqslant 0.109 \pm 0.033$ & -23 & $1 \mathrm{PC}$ \\
\hline 3602 & $5940 \pm 157$ & $\geqslant 6000 \pm 100$ & $0.253 \pm 0.076$ & +24 & $1 \mathrm{PC}$ \\
\hline 3605 & $5126 \pm 152$ & $3600 \pm 150$ & $0.044 \pm 0.018$ & -57 & $1 \mathrm{PC}$ \\
\hline 3606 & $6570 \pm 177$ & $\geqslant 6000 \pm 100$ & $0.399 \pm 0.120$ & +162 & $1 \mathrm{PC}$ \\
\hline 3721 & $6261 \pm 185$ & $\geqslant 6000 \pm 100$ & $0.143 \pm 0.043$ & +51 & $1 \mathrm{PC}$ \\
\hline$\ldots$ & $\ldots$ & $4500 \pm 350$ & $0.016 \pm 0.003$ & -61 & $\ldots$ \\
\hline 3782 & $5018 \pm 250$ & $3400 \pm 135$ & $\geqslant 0.022 \pm 0.011$ & -7 & $1 \mathrm{PC}$ \\
\hline 3837 & $6540 \pm 181$ & $4500 \pm 500$ & $0.048 \pm 0.005$ & +37 & $1 \mathrm{PC}$ \\
\hline 3853 & $5081 \pm 118$ & $3400 \pm 250$ & $0.009 \pm 0.001$ & +22 & $1 \mathrm{PC}$ \\
\hline 3875 & $6022 \pm 198$ & $\geqslant 6000 \pm 100$ & $0.623 \pm 0.187$ & -85 & $1 \mathrm{PC}$ \\
\hline 4345 & $5776 \pm 185$ & $3600 \pm 250$ & $0.012 \pm 0.006$ & -48 & $1 \mathrm{PC}$ \\
\hline 4355 & $5780 \pm \ldots$ & $\geqslant 6000 \pm 850$ & $0.044 \pm 0.013$ & -21 & $1 \mathrm{FP}$ \\
\hline
\end{tabular}


Table 9

(Continued)

\begin{tabular}{|c|c|c|c|c|c|}
\hline \multirow[b]{2}{*}{ KOI } & \multirow{2}{*}{$\begin{array}{c}\text { Primary Star } \\
T_{\text {eff,A }}(\mathrm{K})\end{array}$} & \multicolumn{3}{|c|}{ Companion Parameters } & \multirow{2}{*}{$\begin{array}{c}\text { Planetary } \\
\text { Data }^{a}\end{array}$} \\
\hline & & $T_{\text {eff,B }}(\mathrm{K})$ & $\mathrm{F}_{B} / \mathrm{F}_{A}$ & $\Delta \mathrm{RV}\left(\mathrm{km} \mathrm{s}^{-1}\right)$ & \\
\hline 4457 & $6384 \pm 159$ & $3600 \pm 250$ & $\geqslant 0.012 \pm 0.006$ & +11 & $1 \mathrm{FP}$ \\
\hline 4713 & $5780 \pm \ldots$ & $\geqslant 6000 \pm 100$ & $\geqslant 0.344 \pm 0.103$ & -15 & $1 \mathrm{PC}$ \\
\hline 4871 & $6532 \pm 159$ & $\geqslant 6000 \pm 100$ & $\geqslant 0.012 \pm 0.003$ & -23 & $1 \mathrm{PC}$ \\
\hline
\end{tabular}

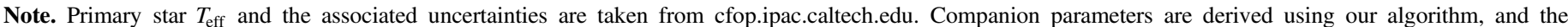

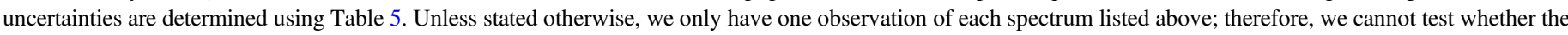
secondary star is part of the bound system or a background star.

${ }^{\mathrm{a}}$ From cfop.ipac.caltech.edu. $\mathrm{PC}$ - planet candidate, $\mathrm{CP}$-confirmed planet, $\mathrm{FP}$-false positive.

temperature of $\sim 3500 \mathrm{~K}$ are consistent with a star whose mass is approximately $0.4 M_{\odot}$.

\section{APPLICATION: 1160 KEPLER OBJECTS}

We have carried out a spectroscopic survey of the $1160 \mathrm{KOI}$ that are brighter than the Kepler magnitude of 14.2, announced as of 2013 September by the Kepler Project and listed in the Exoplanet Archive. ${ }^{4}$ A complete description of the $1160 \mathrm{KOIs}$ and our spectra of them are provided by A. W. Howard et al. (2014, in preparation). We obtained a Keck-HIRES spectrum with an $\mathrm{S} / \mathrm{N}$ of $\sim 45$ per pixel and a resolution of $\mathrm{R}=60,000$ from 3800 to $8000 \AA$ for each one. All spectra are available to the public on the Keck Observatory Archive.

In Table 9 we identify the KOIs where a secondary star was detected by our algorithm, and provide the deduced parameters of both the primary star and the companion. The uncertainties are determined using Table 5, and were computed using the injection-recovery tests. Companion plots for identified KOI binary systems are shown in the Appendix in Figures A1 through A61.

Out of 1160 KOIs, 60 KOIs show clear evidence of companion stars using our spectroscopic algorithm. Additionally, we report three detections that were marginal. Among them, we found $41 \mathrm{KOIs}$ that have one planetary candidate (one validated as a planet), three KOIs that have two planetary candidates, and one KOI with three planetary candidates. In addition, 15 of the 60 KOIs had been marked as false positives in the Exoplanet Archive.

Of course, these spectroscopic detections of secondary stars do not necessarily imply that the planet candidate is false. Instead, each detection merely indicates that there is either a background star or a bound companion to the target KOI. In either case, the transit lightcurve could be caused by a planet transiting the primary star, albeit with a diluted light curve, or an orbiting object that blocks the light of the secondary star, either bound or background.

The relative brightness of the secondary and the primary star can be used to compute the dilution factor of the Kepler light curves. Our wavelength domain approximately encompasses the $\mathrm{V}$ and $\mathrm{R}$ bandpasses of the classical photometry, and the relative brightness can thus be converted to the corresponding value in the Kepler bandpass, and used to determine a more accurate radius for the planet.

Some of the KOIs had secondary stars hotter than the primary star, but contributed only a few percent of the total flux. There are three possible explanations for such cases. First,

\footnotetext{
4 http://exoplanetarchive.ipac.caltech.edu
}

the primary star might have already evolved off main sequence, and become a red giant. In this case, it is possible that it is in fact cooler than the secondary, if the secondary is still on main sequence. The second possible explanation is that only a fraction of the flux from the secondary actually entered the spectrometer slit, and thus not all of the flux from the secondary star is collected by the spectrometer. A third alternative is that the secondary star is simply a background star much further away.

\subsection{KOI Companion Detections}

This section is intended to be used in conjunction with the list of detected companions in Table 9, providing additional information relevant for selected KOIs. We also list the number of confirmed planets and planetary candidates, or indicate whether the KOI was identified as a false positive.

\subsubsection{Confirmed Planets}

KOI-1361-Also known as Kepler-61, it has one confirmed planet (Ballard et al. 2013). We determined the primary star temperature to be $T_{\text {eff }}=4265 \pm 145$, which is $\sim 100 \mathrm{~K}$ larger than Ballard et al. (2013). We identified an even cooler companion, with $T_{\text {eff }}=3600 \pm 130 \mathrm{~K}$, at a radial velocity of $+40 \mathrm{~km} \mathrm{~s}^{-1}$ relative to the primary star. Ballard et al. (2013) reports a companion 0.94 in North of the primary star, detected using adaptive optics. The magnitude difference between the primary star and the companion, as reported by Ballard et al. (2013), is $2.98 \mathrm{mag}$ in Kepler bandpass. This corresponds to the flux ratio of $\mathrm{F}_{A} / \mathrm{F}_{B}$ $=0.064$, which is only slightly above the value deduced by our algorithm, $\mathrm{F}_{A} / \mathrm{F}_{B}=0.022 \pm 0.003$. Some of this difference can be attributed to the difference in the Kepler bandpass regions as compared to the regions used in our algorithm. It is also possible that the spectrometer slit was not necessarily aligned to capture all of the light from the companion, thus the flux ratio is correspondingly lower.

\subsection{Secondary Stars to KOIs with Three Planet Candidates}

KOI-2311-Due to a low RV separation of $+11 \mathrm{~km} \mathrm{~s}^{-1}$, the flux ratio of $0.276 \pm 0.069$ is a lower limit. The final secondary star plot is shown in Figure A26.

\subsubsection{Secondary Stars to KOIs with Two Planet Candidates}

KOI-5-The observed RV trend indicates the possibility of a stellar companion (Wang et al. 2014). Low $\Delta \mathrm{RV}$ of +11 $\mathrm{km} \mathrm{s}^{-1}$ might have caused some of the secondary absorption lines to be subtracted away with the primary star, thus the 
quoted flux ratio of $0.066 \pm 0.020$ is a lower limit rather than an actual value. We analyzed several different observations of the same star, and the RV separation varied with observation time. This indicates that the secondary and the primary star form a bound system. The final companion plot is shown in Figure A1.

KOI-354-Detection of the secondary star to the KOI-354 is marginal, thus we do not list it in Table 9. The system consists of a $5936 \pm 102$ primary star (cfop.ipac.caltech.edu) and an $\mathrm{M}$ dwarf secondary with $T_{\text {eff }}=3500 \pm 250 \mathrm{~K}$. The secondary star has a low relative radial velocity of +11 $\mathrm{km} \mathrm{s}^{-1}$, and thus the flux ratio of $\mathrm{F}_{A} / \mathrm{F}_{B}=0.005 \pm 001$ is a lower limit. The secondary star plot for KOI-354 is shown in Figure A4.

KOI-1613-Due to a low RV separation of $+10 \mathrm{~km} \mathrm{~s}^{-1}$, the flux ratio of $0.044 \pm 0.013$ is a lower limit. Law et al. (2013) detected a secondary star at a separation of $0.22 \mathrm{in}$, which is 1.3 mag fainter than the primary star. A 1.3 magnitude difference indicates a flux ratio of 0.30 , which is much higher than our lower limit. Since they observed the system in a

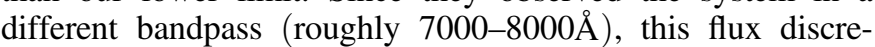
pancy could be due to a different flux ratios in different wavelength regions. However, we suspect that most of the discrepancy can be attributed to the low $\Delta R V$ separation of the two stars of similar spectral types. At $10 \Delta \mathrm{RV}=\mathrm{km} \mathrm{s}^{-1}$, the two sets of absorption lines are barely resolved, and most likely a large fraction of the secondary star was subtracted away together with the primary, leaving only a weak signal in the residuals. The final secondary star plot is shown in Figure A18.

KOI-2059-M dwarf secondary star has a small RV separation of only $+5 \mathrm{~km} \mathrm{~s}^{-1}$, making the flux ratio of 0.016 \pm 0.008 a lower limit. Nevertheless, due to a significantly different secondary spectral type of the secondary compared to the primary with $T_{\text {eff }}$ of $5996 \pm 103 \mathrm{~K}$ (cfop.ipac.caltech.edu), we do not expect the actual flux ratio to deviate significantly from this lower limit. Using AO, a companion was detected at a separation of $0.38 \mathrm{in}, 1.1 \mathrm{mag}$ fainter (Law et al. 2013). A magnitude difference of 1.1 predicts the flux ratio of about 0.36 , which is again larger than the ratio predicted by our algorithm. Again, since the Law et al. (2013) observation was made in the near-infrared region, where the flux of the $M$ dwarfs is considerably larger, we do expect that the flux ratio of an $\mathrm{M}$ dwarf and a $\sim 5000 \mathrm{~K}$ primary star is higher as compared to the ratio in the $\mathrm{V}$ and $\mathrm{R}$ bandpasses. Therefore, our flux ratio is still consistent with the Law et al. (2013) value, especially when accounting for the fact that the ratio predicted by our algorithm as a lower limit. The final secondary star plot is shown in Figure A23.

\subsubsection{Companions to KOIs with One Planet Candidate}

Here we provide notes about selected KOIs that have one planet candidate, and an indication from our spectroscopic analysis of a secondary set of absorption lines in the spectrum. A complete listing of all such detections is in Table 9.

$K O I-151-D u e$ to a low $\triangle \mathrm{RV}$ separation of $+14 \mathrm{~km} \mathrm{~s}^{-1}$, the flux ratio of $0.012 \pm 0.006$ represents a lower limit for KOI151 and its secondary star. The final secondary star plot is shown in Figure A2.

$\mathrm{KOI}-219-$ Both the flux ratio of $0.330 \pm 0.033$ as well as the secondary star $T_{\text {eff }}$ of $6000 \pm 100 \mathrm{~K}$ represent lower limits due to a restricted $T_{\text {eff }}$ library range and low RV separation, respectively. The final secondary star plot is shown in Figure A3.

KOI-652-KOI-652 is at least a triple star system, with a possible marginal detection of the fourth star. All three companions are $\mathrm{M}$ dwarfs, with each contributing only a few percent to the total flux. The final companion plot is shown in Figure A5. In Table 9, we do not list the faintest companion at $-44 \mathrm{~km} \mathrm{~s}^{-1}$, because its detection is only marginal.

KOI-698-This system is analyzed in Santerne (2012). Assuming a main sequence companion, our $T_{\text {eff }}$ estimate for the secondary star of $4800 \pm 600 \mathrm{~K}$ indicates a secondary mass of $0.7-0.8 M_{\odot}$, which is below the predicted value of $1.1 \pm 0.1$ $M_{\odot}$ by Santerne (2012). The final secondary star plot is shown in Figure A6.

KOI-716-The detection of KOI-716 is marginal, and we do not include it in the Table 9. The KOI-716 secondary star is extremely faint and at the threshold of detection, with a flux ratio of $\mathrm{F}_{A} / \mathrm{F}_{B}=0.004 \pm 0.001$. The primary star is a $T_{\text {eff }}$ $=6096 \pm 150 \mathrm{~K}$ (cfop.ipac.caltech.edu), and the secondary star is an $\mathrm{M}$ dwarf with $T_{\text {eff }}=3500 \pm 250 \mathrm{~K}$. Due to a relatively low RV separation of $+18 \mathrm{~km} \mathrm{~s}^{-1}$, the flux ratio might be underestimated, yet we do not expect the secondary star contribution to be larger than $1 \%$ of the total flux. KOI-716 has one planetary candidate, and the secondary star plot is shown in Figure A7.

KOI-984-AO imaging of KOI-984 revealed a companion of approximately equal magnitude 1.5 arcsec apart (Law et al. 2013). Our algorithm, however, detects a marginal secondary star at $\Delta \mathrm{RV}=+33 \mathrm{~km} \mathrm{~s}^{-1}$ with $T_{\text {eff }}=3500 \pm 150 \mathrm{~K}$, contributing less than $1 \%$ to the total flux. This suggests that only a small fraction of the light from a secondary star star actually entered the slit, and while we can confirm the possibility of the companion, its estimated parameters are highly inaccurate. The final secondary star plot is shown in Figure A9. Since the detection is marginal, we do not list it in Table 9.

KOI-1020 - The existence of the companion already speculated by (Hirano et al. 2012). The final secondary star plot is shown in Figure A10.

KOI-1137-Despite low RV separation of $+11 \mathrm{~km} \mathrm{~s}^{-1}$, the two stars are of sufficiently distinct spectral types that we do not expect the flux ratio to be significantly higher than the calculated lower limit of $0.020 \pm 0.004$. The final secondary star plot is shown in Figure A12.

KOI-1452 - The primary star has $T_{\text {eff }}=7172 \pm 211 \mathrm{~K}$ (cfop. ipac.caltech.edu), which is outside our library range. While the binarity of the spectrum is clear, the parameters for the secondary star cannot be determined accurately enough to be published. This is mostly due to a high rotational broadening of the secondary absorption lines, indicating a secondary star with a spectral type outside the range of our SpecMatch library. The final secondary star plot is shown in Figure A17.

$K O I-1784$-Due to a low RV separation of $-13 \mathrm{~km} \mathrm{~s}^{-1}$, the flux ratio of $0.192 \pm 0.058$ represents a lower limit. The final secondary star plot is shown in Figure A21.

$\mathrm{KOI}-2075$ - The secondary star is faint, contributing less than $1 \%$ to the total flux. While the flux ratio of $0.008 \pm 0.004$ is a lower limit due to a low RV separation of $-13 \mathrm{~km} \mathrm{~s}^{-1}$, we do not expect that a large fraction of the secondary absorption lines got subtracted away together with the primary star, as the 
two spectral types differ significantly from each other. The final secondary star plot is shown in Figure A24.

KOI-2215-This is a triple-lined spectrum, with both secondary and tertiary stars clearly detectable. Both additional stars are treated separately, but the uncertainties on the parameters might be slightly higher due to the blending of spectral lines as the spectral types of all three stars are relatively similar. The flux ratio for the third star of $0.155 \pm 0.047$ is a lower limit due to a low RV separation of $-15 \mathrm{~km} \mathrm{~s}^{-1}$. The final secondary star plot is shown in Figure A25.

KOI-2457-The primary star effective temperature of 6728 \pm 155 (cfop.ipac.caltech.edu), exceeds our temperature range of the SpecMatch library. This hinders our ability to determine accurate parameters for the secondary star at a low $\Delta \mathrm{RV}$ separation of $+14 \mathrm{~km} \mathrm{~s}^{-1}$. Nevertheless, our analysis indicates a secondary star with a lower limit flux ratio of $0.076 \pm 0.023$. The final secondary star plot is shown in Figure A27.

KOI-3471-The spectrum of KOI-3471 contains four stars, all but one of comparable brightness. We provide estimates for the parameters for each of the constituent stars, but expect larger errors due to significant blending of their spectral lines. Since all of the stars are contained within less than $80 \mathrm{~km} \mathrm{~s}^{-1}$ $\Delta \mathrm{RV}$, we expect that the temperatures and the flux ratios for additional stars are affected by the presence of the other stars. The final plot is shown in Figure A41.

KOI-3573-Low RV separation of $-15 \mathrm{~km} \mathrm{~s}^{-1}$, together with the similar spectral types of the two stars, suggest that the actual flux ratio is probably higher than the calculated lower limit of $0.109 \mathrm{lpm} 0.033$. As the two sets of absorption lines overlap slightly, it is likely that a fraction of the secondary absorption lines got subtracted away together with the primary star, thus making the secondary star appear fainter. The final secondary star plot is shown in Figure A47.

KOI-3583 - The secondary star $\chi^{2}$ minimum is significantly broadened, indicating rotational broadening of secondary star absorption lines. Thus, despite the RV separation of -23 $\mathrm{km} \mathrm{s}^{-1}$, there is a possibility for the blending of the two sets of absorption lines, and the calculated flux ratio of $0.109 \pm 0.033$ is a lower limit. The final secondary star plot is shown in Figure A48.

KOI-3606-Both the secondary and the primary $\chi^{2}$ minimum are broadened, indicating a significant rotational broadening of the spectral lines. As this is characteristic of hot stars, we assume that the actual $T_{\text {eff }}$ for the secondary star is above the quoted lower limit of $6000 \pm 100 \mathrm{~K}$. The final secondary star plot is shown in Figure A51.

KOI-3721-The spectrum of KOI-3721 contained three sets of absorption lines, with the faintest star contributing only $\sim 1 \%$ to the total flux. The brighter secondary star $\chi^{2}$ is broad, indicating significant rotational broadening. This is consistent with the estimate of the secondary star $T_{\text {eff }}$ above $6000 \pm$ $100 \mathrm{~K}$. The final plot is shown in Figure A52.

$\mathrm{KOI}-3782$ - The calculated flux ratio is a lower limit due to a low RV separation of $-7 \mathrm{~km} \mathrm{~s}^{-1}$, but we do not expect a significant deviation from the stated flux ratio due to significantly different spectral types of the two stars. The final secondary star plot is shown in Figure A53.

KOI-4713-Due to similar spectral types of the two stars, as well as the small RV separation of $-15 \mathrm{~km} \mathrm{~s}^{-1}$, the calculated flux ratio of $0.344 \pm 103$ is a lower limit. The final secondary star plot is shown in Figure A60.
KOI-4871-Despite the RV separation of $-23 \mathrm{~km} \mathrm{~s}^{-1}$, we state the flux ratio of $0.012 \pm 0.003$ as the lower limit due to the similarity of the primary and secondary star spectral type. This similarity allows for a chance of overlap of some of the absorption lines, and some of the secondary absorption lines might have been subtracted away with the primary star. The final secondary star plot is shown in Figure A61.

\subsubsection{Confirmed False Positives}

KOI-1152-KOI-1152 has a known companion that was previously detected using AO (Law et al. 2013). The planet candidate was identified as a false positive (cfop.ipac.caltech. edu). We analyzed several different observations of the same star, and the RV separation varied with different observation times. This indicates that the secondary and the primary star form a bound system. The final secondary star plot is shown in Figure A13.

KOI-3000 - The flux ratio of $0.055 \pm 0.019$ is a lower limit rather than an actual value due to a low RV separation of +10 $\mathrm{km} \mathrm{s}^{-1}$. Nevertheless, the two stars have sufficiently different spectral types that we do not expect a significant departure from the stated flux ratio. The final secondary plot is shown in Figure A32.

KOI-3035-Despite moderate RV separation of $+20 \mathrm{~km} \mathrm{~s}^{-1}$, both sets of spectral lines are rotationally broadened, causing the two sets of absorption lines to overlap slightly. Therefore, the flux ratio of $0.212 \pm 0.064$ is a lower limit rather than an actual value. The final secondary star plot is shown in Figure A34.

KOI-3162 - The flux ratio of $0.553 \pm 0.166$ is a lower limit due to a low RV separation of $-13 \mathrm{~km} \mathrm{~s}^{-1}$. The final secondary star plot is shown in Figure A36.

KOI-3216-Flux ratio of $0.326 \pm 0.033$ is a lower limit due to a low RV separation of $+13 \mathrm{~km} \mathrm{~s}^{-1}$. The final secondary star plot is shown in Figure A37.

KOI-4355-The secondary star to KOI-4355 exhibited a broad minimum, indicating significant rotational broadening. Due to shallower spectral lines, the uncertainties on the secondary star parameters might be somewhat larger. The final secondary star plot is shown in Figure A58.

$\mathrm{KOI}-4457$ - The calculated flux ratio of $0.012 \pm 0.003$ is a lower limit due to a low RV separation of $-15 \mathrm{~km} \mathrm{~s}^{-1}$, but we do not expect significant deviation from the stated value due to the significantly different spectral types of the two stars. The final secondary star plot is shown in Figure A59.

\section{LIMITATIONS}

\subsection{Relative Radial Velocity for the Secondary Star}

Our code cannot detect secondary stars with $|\Delta \mathrm{RV}|<10$ $\mathrm{km} \mathrm{s}^{-1}$, with the exception of $\mathrm{M}$ dwarfs. For bound systems consisting of two solar type stars, this corresponds to the physical separation of at most $\sim 5 \mathrm{AU}$, assuming the orbital plane is viewed edge-on and that the spectrum was taken when the two stars were coming directly toward or away from us. On the other hand, if the phase of the two stars is such that their momentary velocity is close to tangential, our code will not be able to detect the presence of the secondary star, regardless of their relative brightness. Somewhat less strictly, we are also limited to orbits with periods larger than $\sim 2.5$ days, as the maximum detectable Doppler shift for the secondary star is at $\pm 200 \mathrm{~km} \mathrm{~s}^{-1}$. This limit is determined 
assuming that the two stars in the binary system are solar type, that the spectrum was taken when the two stars were coming directly toward or away from us, and that the orbit is viewed edge-on. Shorter orbital periods can thus be detected for binaries with lower mass companions, for orbits viewed at an angle, or when the spectrum was taken at a different orbital phase.

For both bound companions and background secondary stars, whenever the spectral types of the two stars are similar and their relative radial velocity is low $\left(\lesssim 20 \mathrm{~km} \mathrm{~s}^{-1}\right.$, with the exact number depending upon the amount of the absorption line broadening in the spectrum), there is a possibility that some of the absorption lines of the secondary spectrum get subtracted away together with the primary star. In those cases, the flux of the secondary star is somewhat underestimated, and its parameters carry somewhat larger uncertainties.

\subsection{Effective Temperature}

The SpecMatch library used for our algorithm contains stars with $T_{\text {eff }}$ from 3200 to $6550 \mathrm{~K}$. For detecting the secondary star, the $T_{\text {eff }}$ range is slightly narrower, ranging from 3300 to $6100 \mathrm{~K}$. This is due to the fact that we only construct the median library spectrum for a particular $100 \mathrm{~K}$ $T_{\text {eff }}$ range when there are at least three different SpecMatch spectra within that range. For any primary star with $T_{\text {eff }}$ outside the SpecMatch library range, the fit will not be exact, and the subtraction will be imperfect-this decreases the detectability of the secondary stars with $\Delta \mathrm{RV} \lesssim 10 \mathrm{~km} \mathrm{~s}^{-1}$. Furthermore, for any secondary star with $T_{\text {eff }}$ that is much lower than $3300 \mathrm{~K}$ or much higher than $6100 \mathrm{~K}$, we cannot accurately estimate the percentage flux because the injected spectrum cannot match the actual secondary star spectrum. As a result, deduced $T_{\text {eff }}$ and flux contribution for stars outside the SpecMatch parameter range carry larger uncertainties. Nevertheless the binarity of the spectrum can still be established with a sufficient degree of certainty (see KOI-54 example in Section 4.2.6).

Since we cannot extrapolate the $\chi^{2}$ minima distribution function to temperatures lower than $3300 \mathrm{~K}$, we cannot predict whether a secondary star whose effective temperature is estimated at $\sim 3300 \mathrm{~K}$ is actually cooler than this limiting temperature. Thus, the $3300 \mathrm{~K}$ secondary star $T_{\text {eff }}$ is always more of an upper limit than an actual value. Similarly, we cannot extrapolate the behavior of the $\chi^{2}$ minima distribution to effective temperatures higher than $\sim 6000 \mathrm{~K}$, and any $\sim 6100 \mathrm{~K}$ estimated secondary star $T_{\text {eff }}$ is a thus a lower limit rather than the actual estimate.

\subsection{Rotational Broadening}

Rotational broadening does not pose a major challenge to the identification of the primary star, because we artificially broaden the library spectrum's absorption lines to match the amount of broadening present in the spectrum. We can fit and subtract the primary absorption lines successfully regardless of the star's rotation. For the secondary star, however, any significant broadening of absorption lines impairs our ability to detect it. As the lines become broader, they become shallower, and thus less distinct from the noise. This also causes a less sharp and distinct residual $\chi^{2}$ minimum that cannot be as easily distinguished from accidental alignments.
For cases where the secondary star has a significant rotational broadening but is sufficiently bright to enable detection, the estimated secondary temperature and its flux contribution become more uncertain. Since we do not artificially broaden the lines of the median library spectra used for the search of the secondary star, even the spectrum with $T_{\text {eff }}$ matching that of the actual secondary star is a poor match. Thus, because none of the median library spectra match the secondary star well, the differences in the $\chi^{2}$ minima are smaller among the $T_{\text {eff }}$ values for the median library spectra, and the residual $\chi^{2}$ minimum distribution's minimum is harder to identify correctly. Furthermore, shallower absorption lines cause the underestimation of the flux contribution, and the flux ratio for the two stars is also underestimated.

\subsection{Fractional Flux Contribution from the Secondary Star}

For the secondary star to be detectable, its fractional contribution most often needs to reach a value that is at least one half of the noise; for example, a $1 \%$ flux from the secondary star for a spectrum with $2 \%$ can be detected. This brings the $\mathrm{S} / \mathrm{N}$ of the residuals after the subtraction of the primary star to 0.5 . The exact value also depends on the spectral differences between the two stars, as well as their relative radial velocity.

At the other end, secondary stars with fractional contributions that are comparable to that of their primary stars also pose a challenge. While those stars are easily detectable, the determination of the properties for the secondary star is limited by the pollution of one set of absorption lines by the other. This effect is especially apparent when their relative radial velocity is low, with one set of lines significantly encroaching on the other. In those cases, the primary star fit and the secondary star parameters suffer by this mutual pollution of their respective absorption lines. Since some of the secondary absorption lines get subtracted away together with the primary star, this also causes the calculated flux ratio for the two stars to be below the true value.

\section{CONCLUSION}

Using the algorithm described in this paper we were able to detect secondary stars in the Keck-HIRES spectra that contributed as low as $1 \%$ of the total flux in the spectrum with a 2\% Poisson noise. These secondary stars can be angularly separated from the primary star by at most 0.43 in $-1.5 \mathrm{in}$, depending on the orientation of the aperture slit. For detection, the relative radial velocity for the two stars needs to exceed 10 $\mathrm{km} \mathrm{s}^{-1}$, except for the case of the G-type primary star and an M dwarf. For G-M pairs there is no restriction on the radial velocity separation for the two stars, given that the two sets of absorption lines are not overlapping exactly; for these, we were able to detect secondaries as faint as $0.5 \%$ relative to the primary star in a spectrum with a $2 \%$ Poisson noise. Our results suggest the detectability threshold $\mathrm{S} / \mathrm{N}$ for the secondary star of 0.5 for any spectral type combination of the two stars, and 0.25 for $\mathrm{M}$ dwarf secondaries to G-type primary stars.

For cases where the secondary star is detected, its effective temperature and the fractional flux contribution can be determined. The uncertainties in the derived parameters depend on the relative brightness of the secondary star, as well as on the spectral types of the two stars in the spectrum. These values 
are summarized in Table 5. For the cases of non-detections, we place an upper limit for the brightness of any potential hidden companions.

Currently, we are limited to stars with the effective temperature above $3300 \mathrm{~K}$ and below $6500 \mathrm{~K}$; for the stars outside this temperature range, the presence of the sufficiently bright secondary star can still be evaluated, but the derived parameters carry larger uncertainties. The parameter errors also increase when the two stars have comparable flux contributions, or when their spectral types are similar. Our results are most accurate for the cases where the relative radial velocity of the two stars exceeds $10 \mathrm{~km} \mathrm{~s}^{-1}$, both stars have $T_{\text {eff }}$ between 3000 and $6000 \mathrm{~K}$, and the secondary star contributes less than $20 \%$ of the total flux for the system. The recovery rate for a given radial velocity separation and relative brightness of the secondary star is the highest for $\mathrm{M}$ dwarfs, given that the primary star is of a different spectral type.

This spectroscopic algorithm can be useful for transiting planets toward recognizing multiple potential host stars for the planet and for computing photometric dilution in the analysis of the planet. As an application of the algorithm, we analyzed $1160 \mathrm{KOI}$ and found evidence of at least one additional star in 63 KOIs. A secondary star does not imply that the planet is false, but merely indicates that there is another star present that is angularly close to the primary star, either bound or background. Our estimate of the fractional flux contribution from the secondary star for each of these 63 KOIs can be used to compute the dilution factor for their Kepler light curves, and help to determine a more accurate radius of the planet.

We thank John Johnson for the CalTech Keck Telescope time to obtain many of the spectra of the KOIs. We also thank John Brewer and Debra Fischer for their preliminary estimates of the stellar parameters for the Specmatch library stars, as their parameters are an improvement over the values in Valenti \& Fischer (2005), with a published paper in preparation. G. Marcy, the Alberts Chair at UC Berkeley, would like to thank Marilyn and Watson Alberts for funding that made this research possible. We also thank the John Templeton Foundation and NASA grant NNX11AK04A for funding this research. We thank the government of the Republic of Slovenia and its Slovene Human Resources and

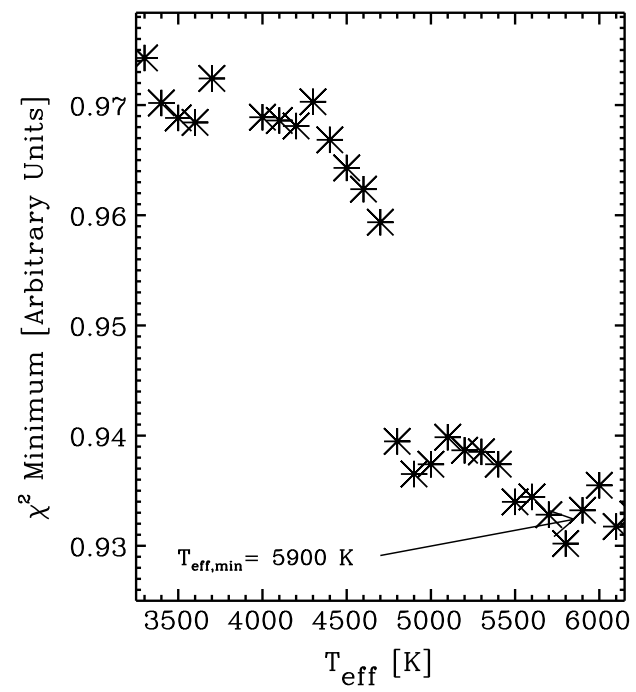

Scholarship Fund and its Ad futura programme that helps fund R. Kolbl.

Kepler was competitively selected as the 10th NASA Discovery mission. Funding for this mission is provided by the NASA Science Mission Directorate. Some of the data presented herein were obtained at the W. M. Keck Observatory, which is operated as a scientific partnership among the California Institute of Technology, the University of California, and the National Aeronautics and Space Administration. The Keck Observatory was made possible by the generous financial support of the W. M. Keck Foundation.

The authors would like to thank the many people who gave so generously of their time to make the NASA Kepler mission a success. All spectra used in this paper are available at the Keck Observatory Archive. All Kepler data products are available to the public at the Mikulski Archive for Space Telescopes http://stdatu.stsci.edu/kepler and the spectra and their products are made available at the NExSci Exoplanet Archive and its CFOP website: http://exoplanetarchive. ipac.caltech.edu We thank the many observers who contributed to the measurements reported here. We gratefully acknowledge the efforts and dedication of the Keck Observatory staff, especially Scott Dahm, Hien Tran, and Grant Hill for support of HIRES, and Greg Wirth for support of remote observing. This work made use of the SIMBAD database (operated at CDS, Strasbourg, France) and NASA's Astrophysics Data System Bibliographic Services. This research has made use of the NASA Exoplanet Archive, which is operated by the California Institute of Technology, under contract with the National Aeronautics and Space Administration under the Exoplanet Exploration Program. Finally, the authors wish to extend special thanks to those of Hawaiian ancestry on whose sacred mountain of Mauna Kea we are privileged to be guests. Without their generous hospitality, the Keck observations presented herein would not have been possible.

\section{APPENDIX}

Graphical supporting evidence for KOIs that showed an evidence of secondary star, listed in Table 9 are shown in Figures A1 - A61.

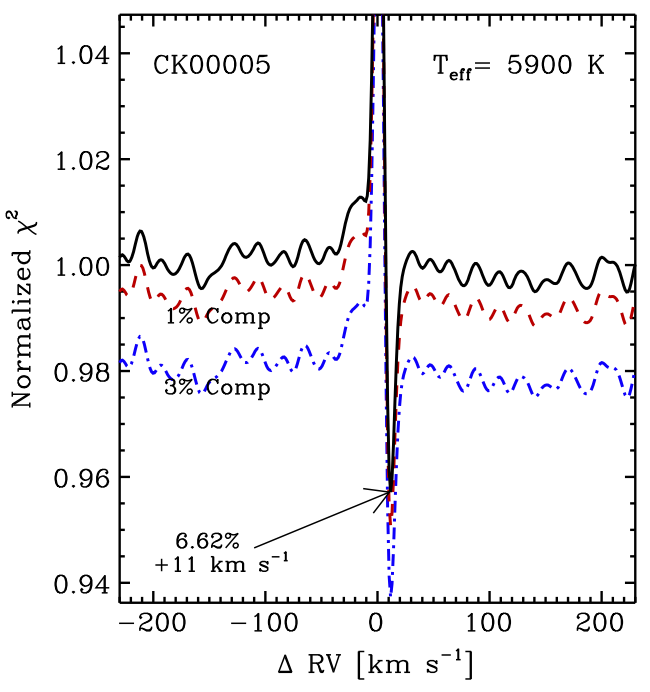

Figure A1. Final secondary star plot for KOI-5. Same as Figure 9. 

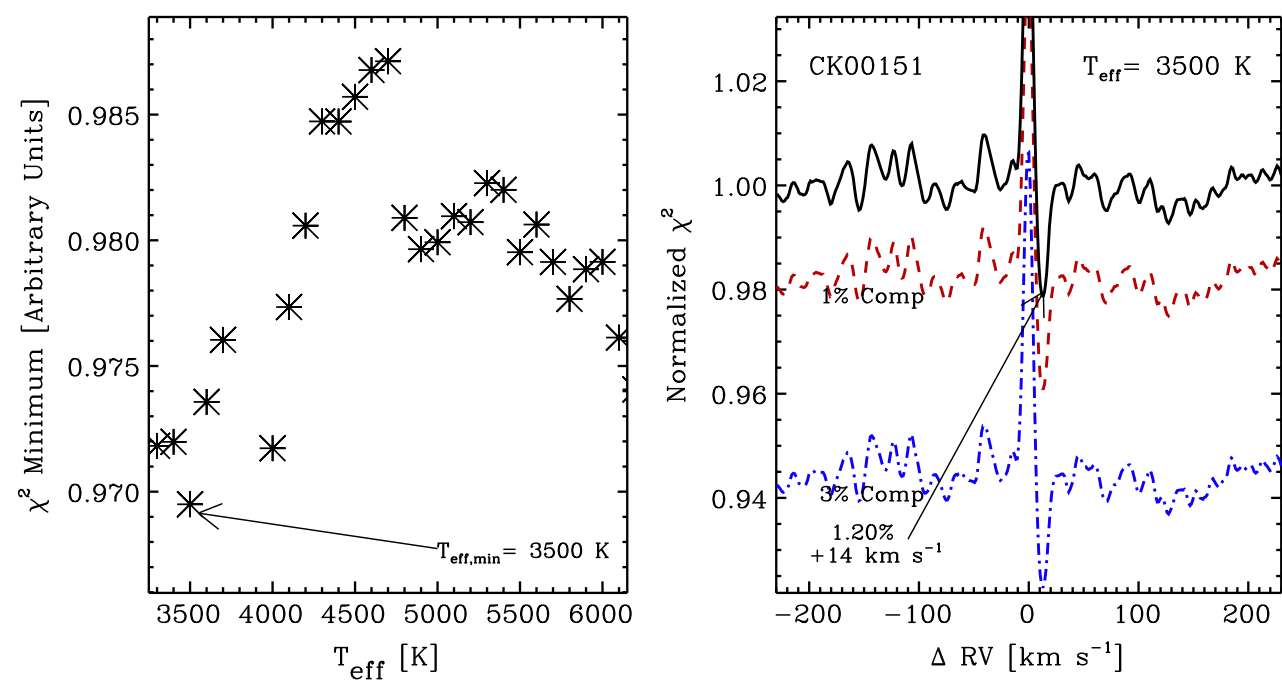

Figure A2. Final secondary star plot for KOI-151. Same as Figure 9.
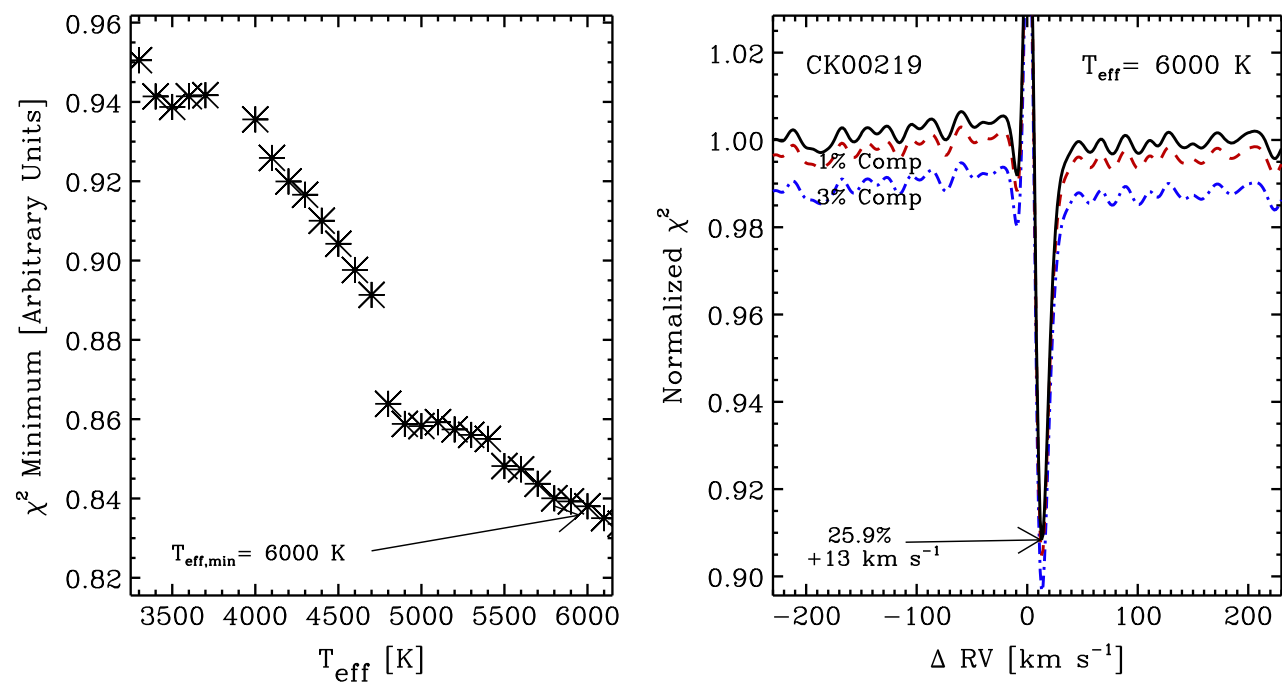

Figure A3. Final secondary star plot for KOI-219. Same as Figure 9.
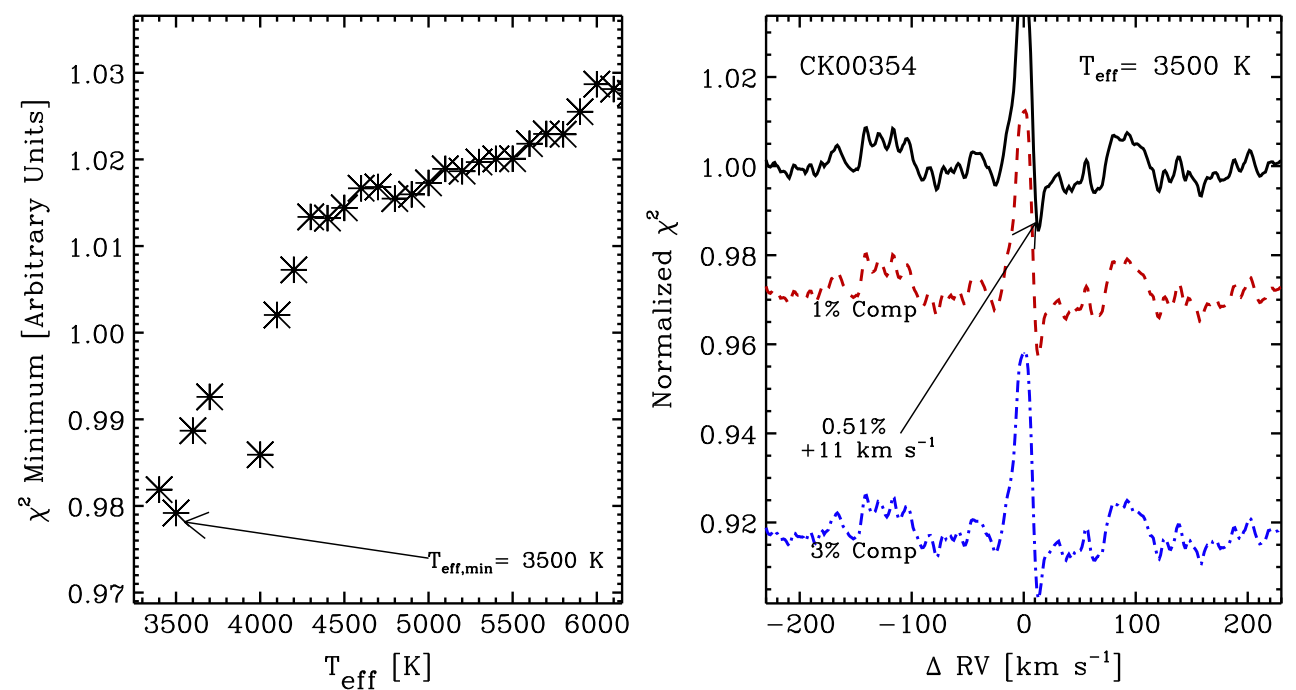

Figure A4. Final secondary star plot for KOI-354. Marginal detection. Same as Figure 9. 

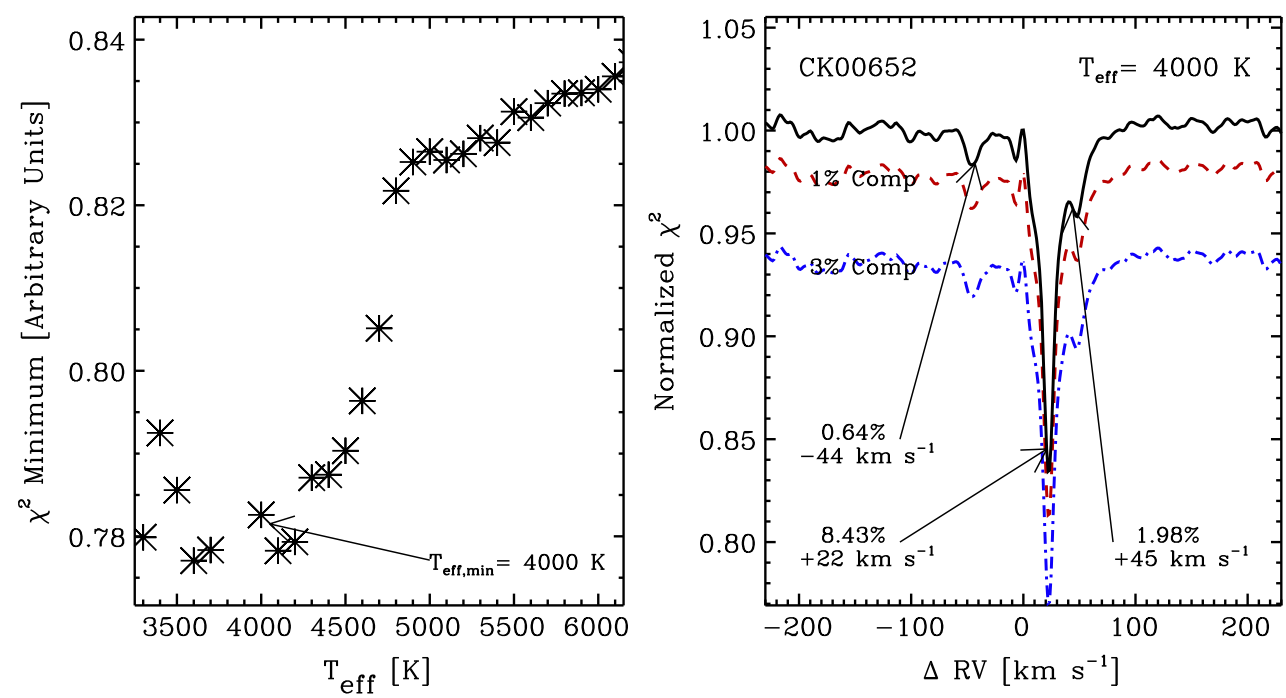

Figure A5. Final secondary star plot for KOI-652. Same as Figure 9. At left, we only show the $\chi^{2}$ minimum function for the brightest companion. At right, all three companions are annotated with an arrow. The detection of the faintest companion, contributing $0.6 \%$ to the total flux, is marginal and thus not listed in Table 9 .
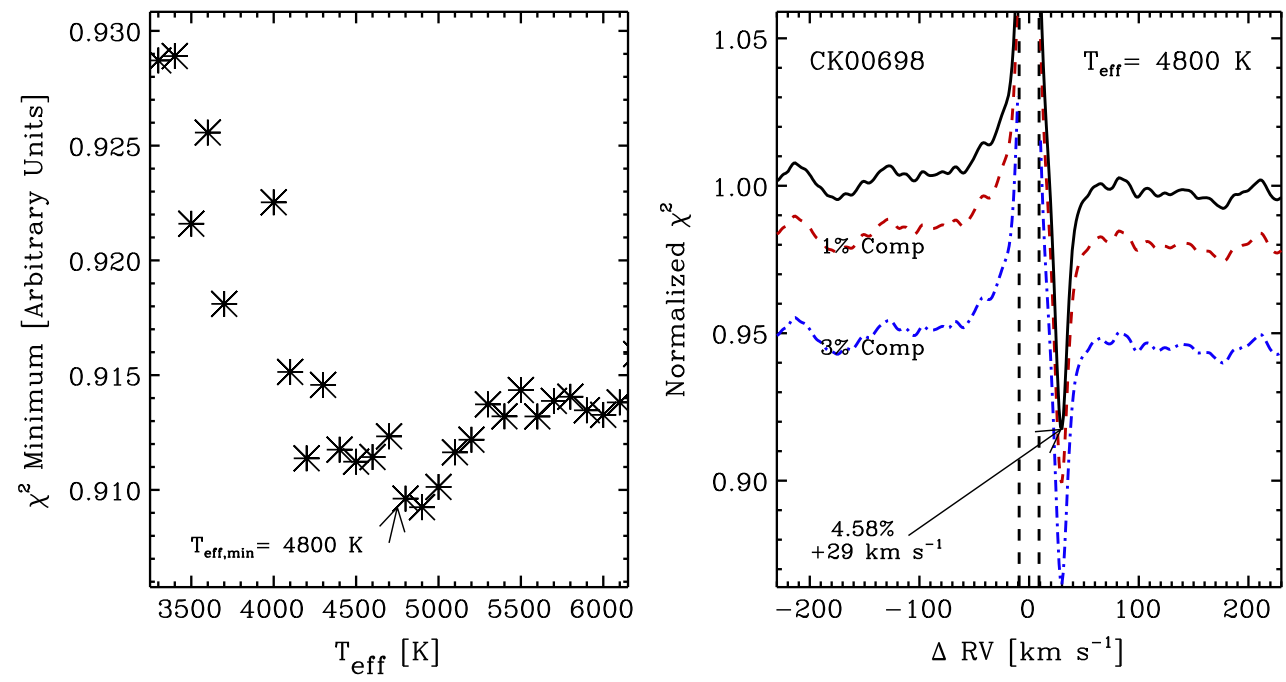

Figure A6. Final secondary star plot for KOI-698. Same as Figure 9.
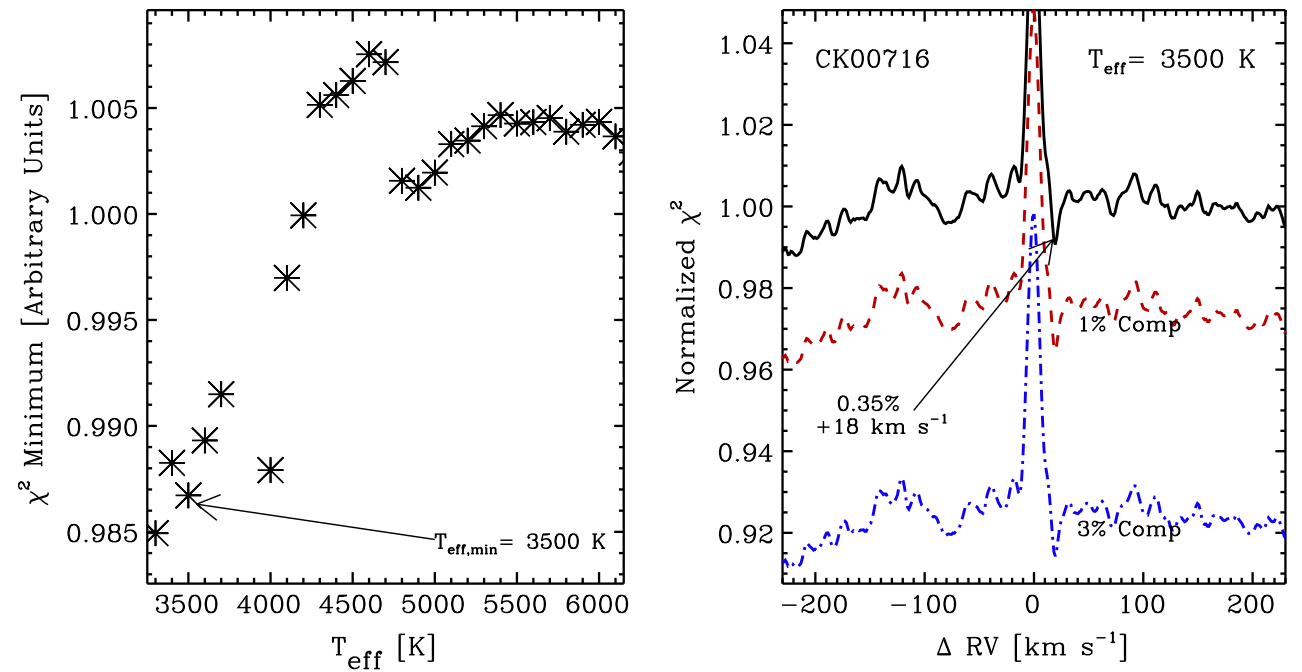

Figure A7. Final secondary star plot for KOI-716. Marginal detection. Same as Figure 9. 

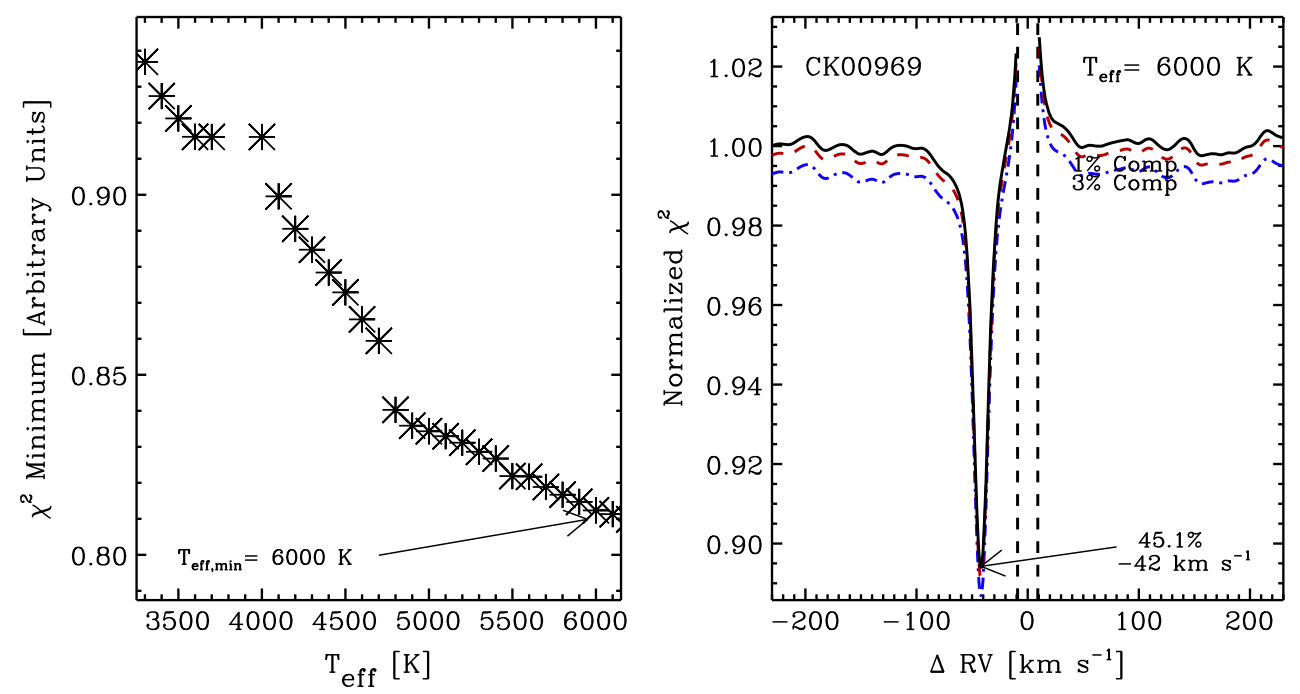

Figure A8. Final secondary star plot for KOI-969. Same as Figure 9.
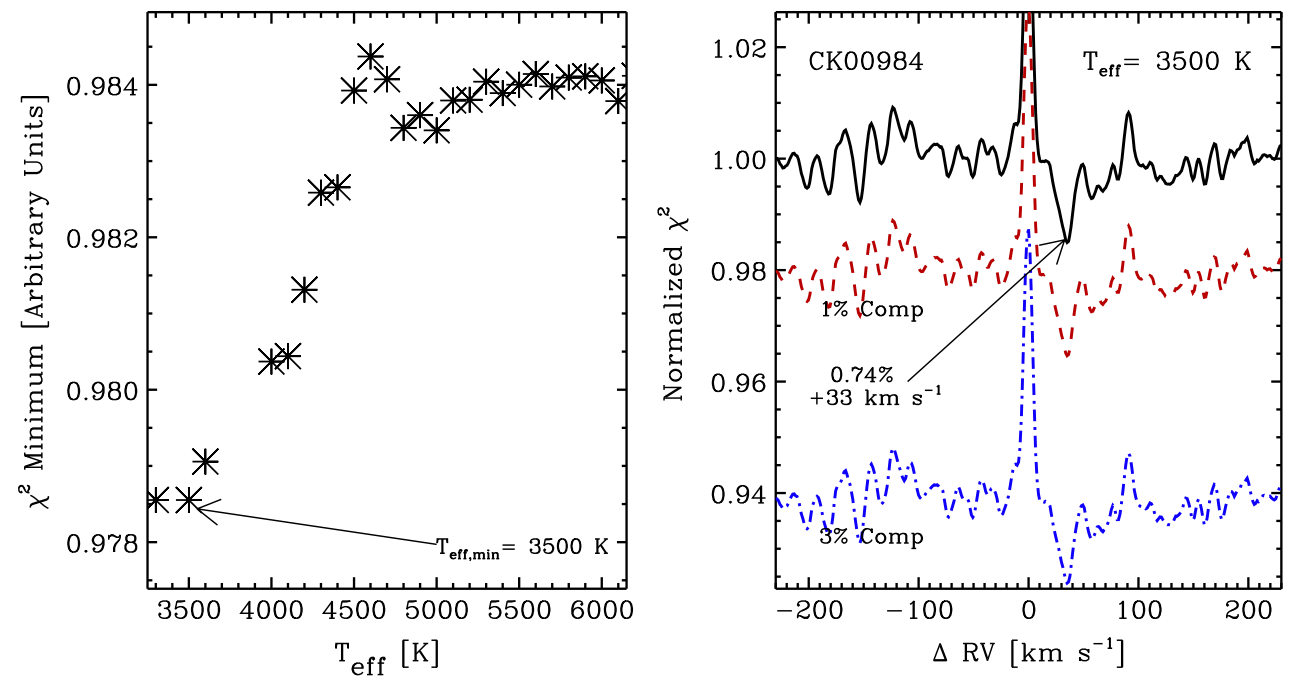

Figure A9. Final secondary star plot for KOI-984. Same as Figure 9.
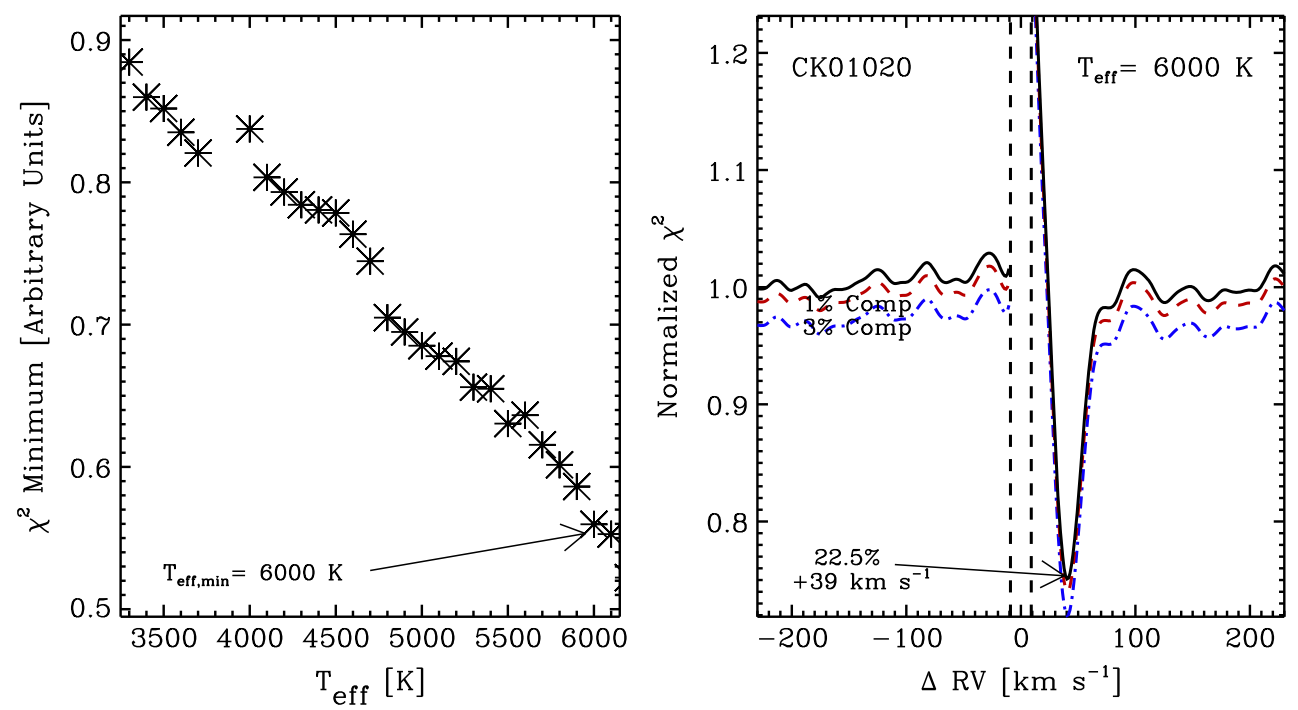

Figure A10. Final secondary star plot for KOI-1020. Same as Figure 9. 

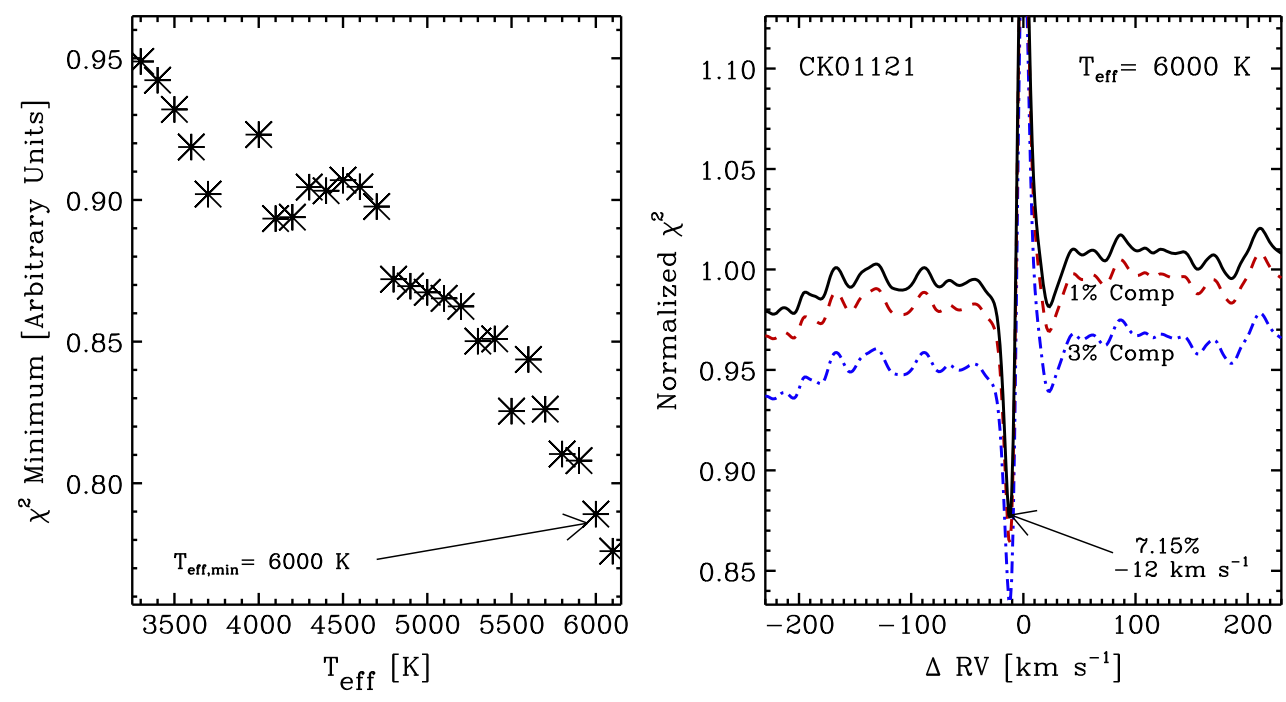

Figure A11. Final secondary star plot for KOI-1121. Same as Figure 9.
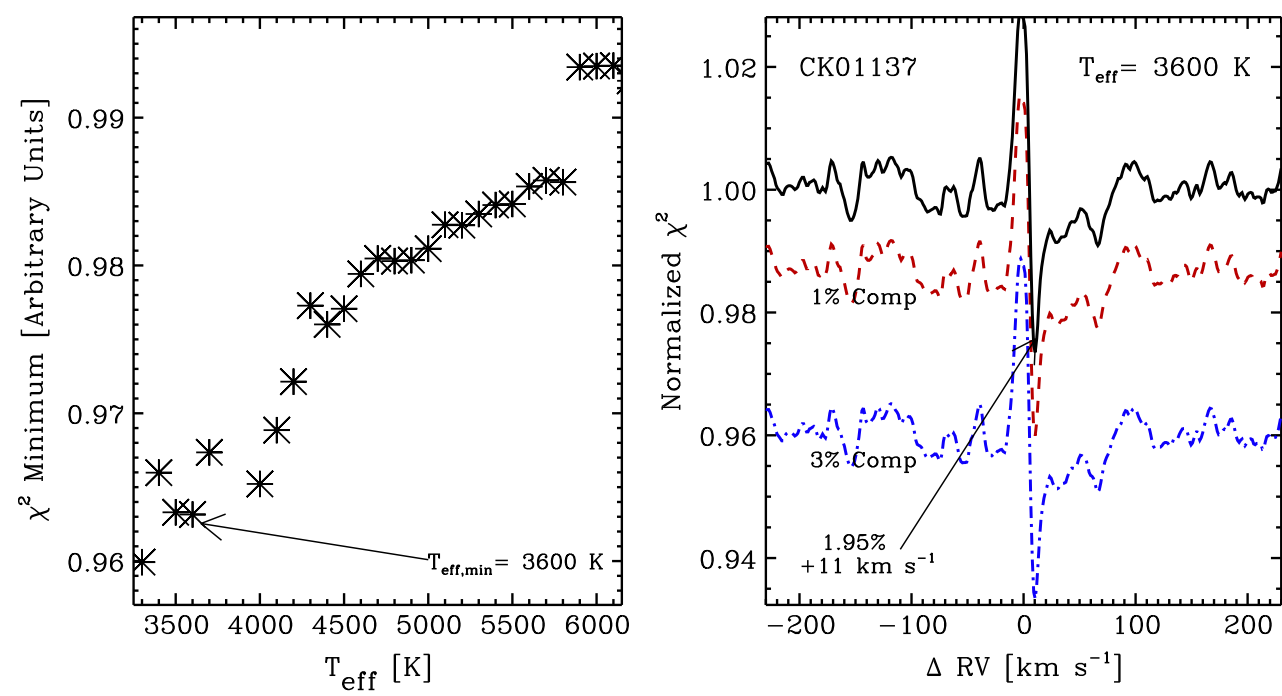

Figure A12. Final secondary star plot for KOI-1137. Same as Figure 9.
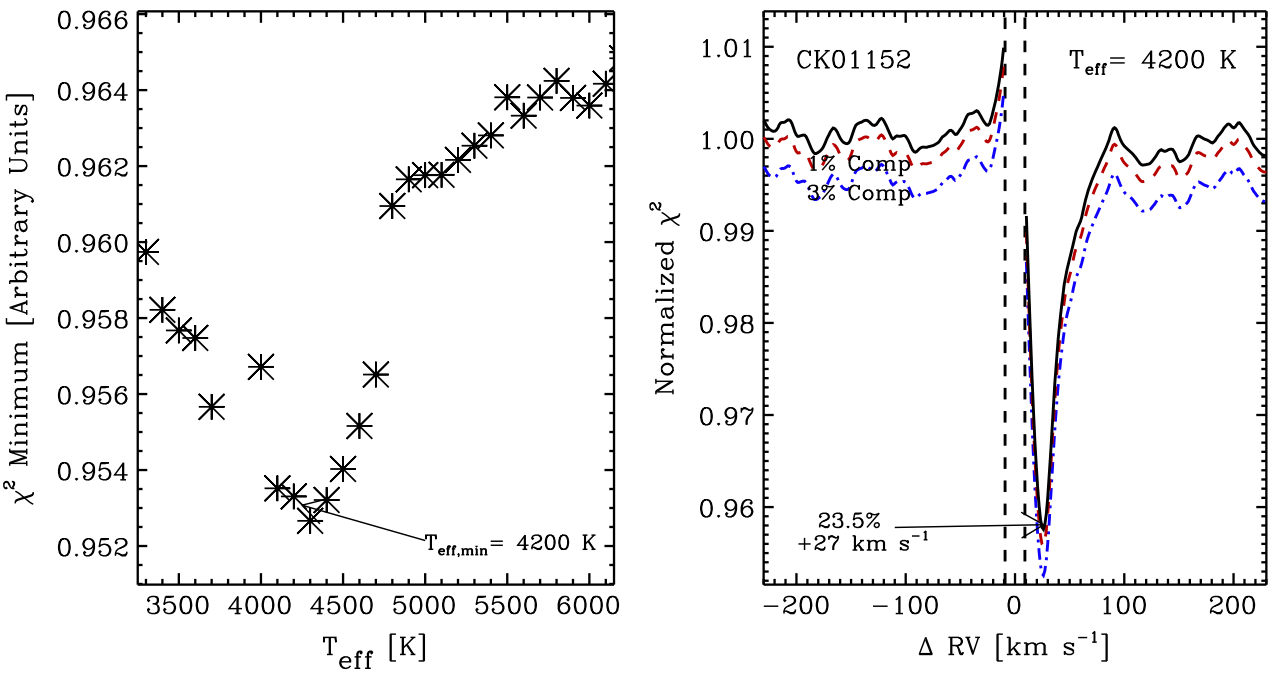

Figure A13. Final secondary star plot for KOI-1152. Same as Figure 9. 

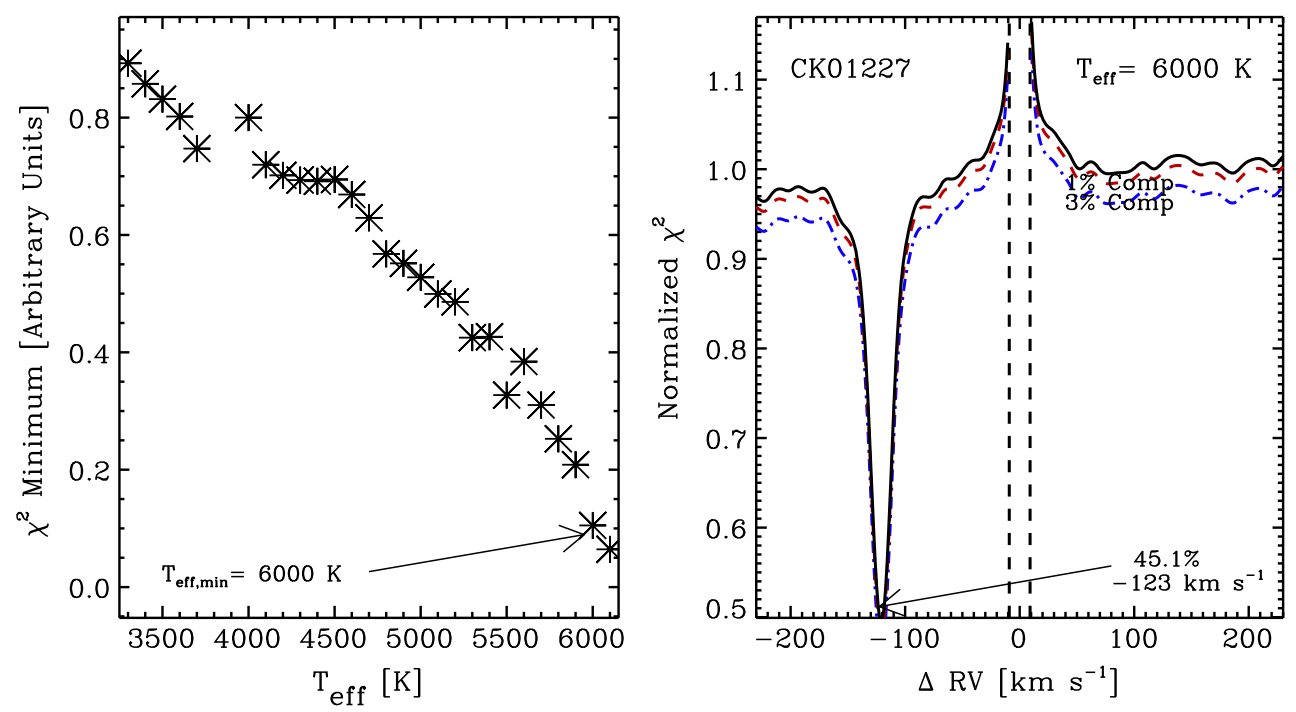

Figure A14. Final secondary star plot for KOI-1227. Same as Figure 9.
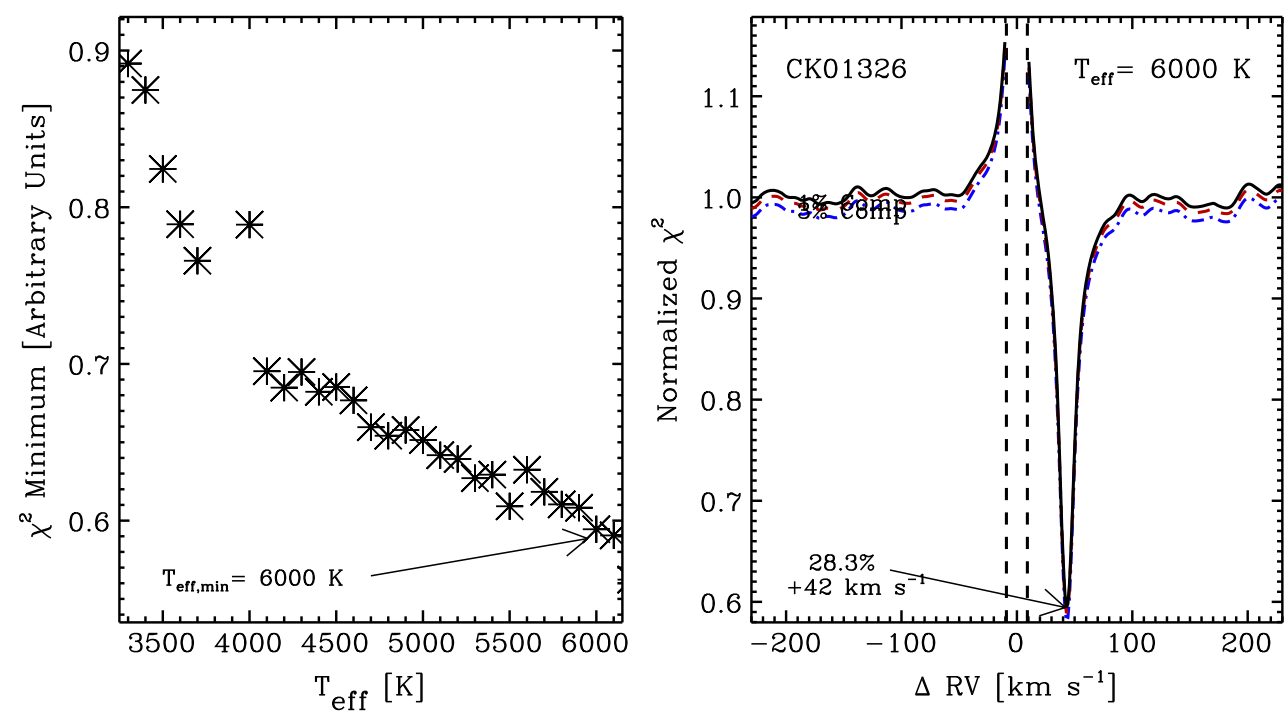

Figure A15. Final secondary star plot for KOI-1326. Same as Figure 9.
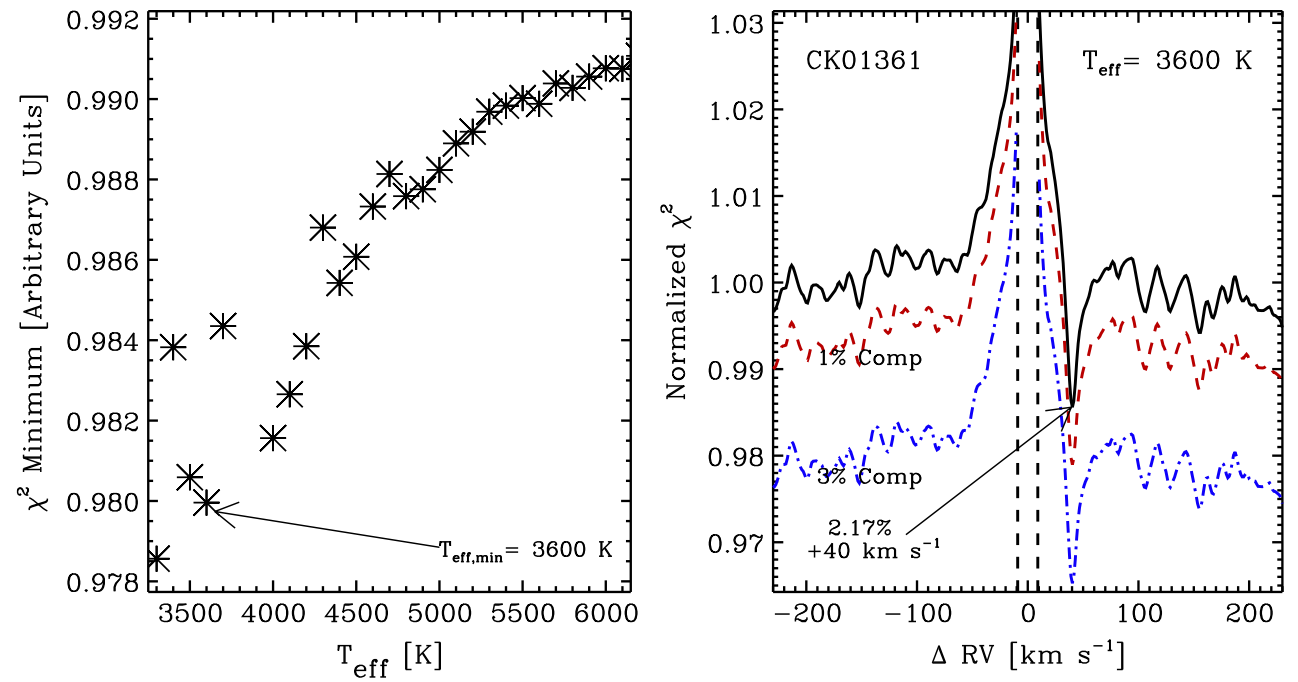

Figure A16. Final secondary star plot for KOI-1361. Same as Figure 9. 

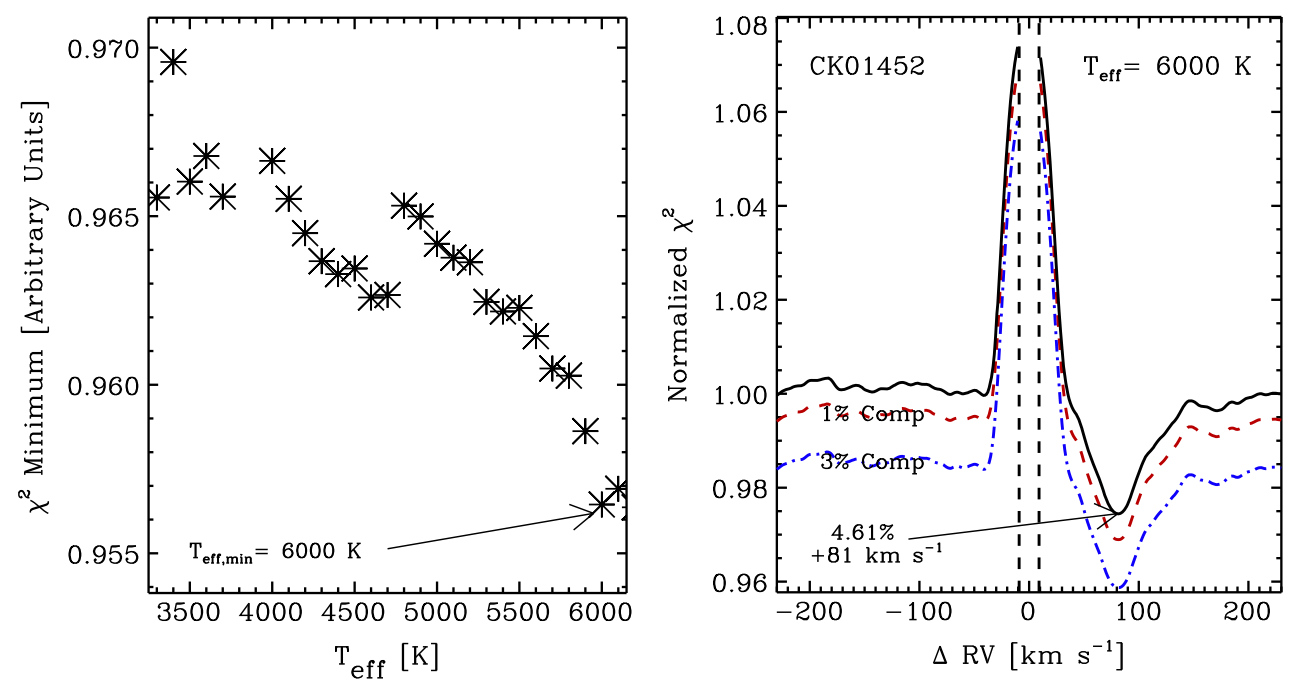

Figure A17. Final secondary star plot for KOI-1452. Same as Figure 9.
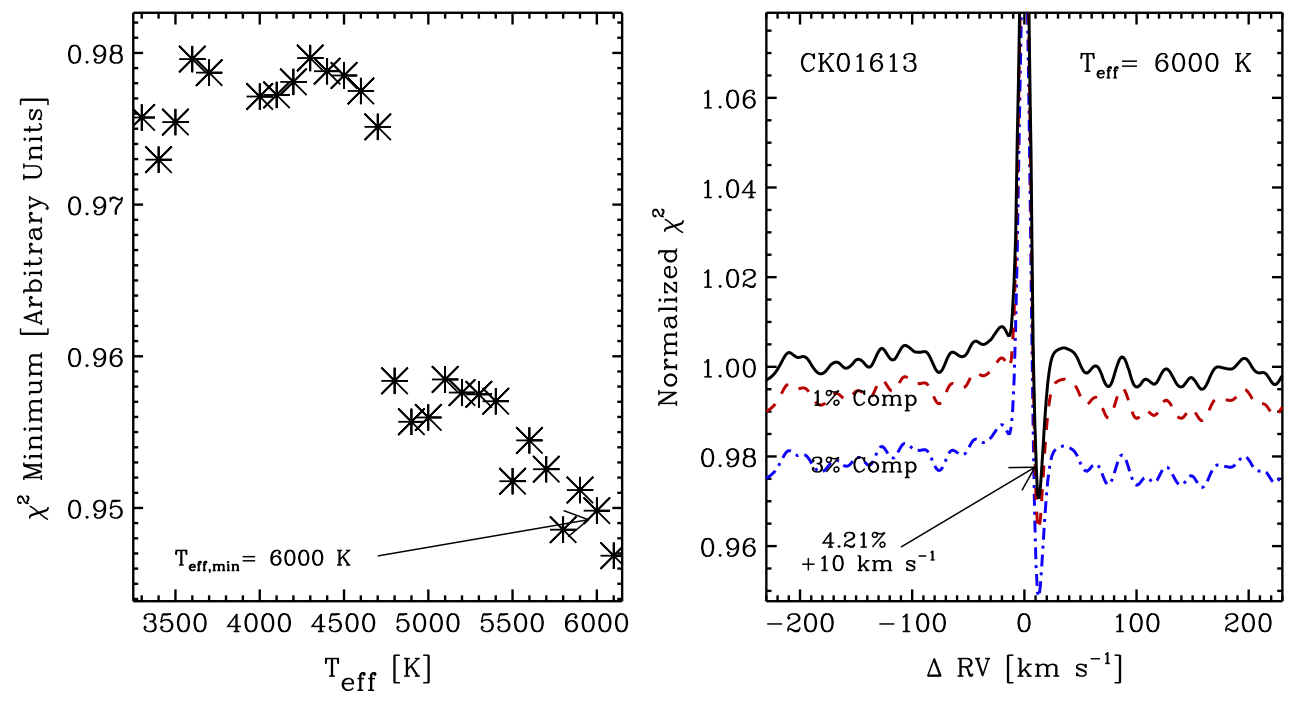

Figure A18. Final secondary star plot for KOI-1613. Same as Figure 9.
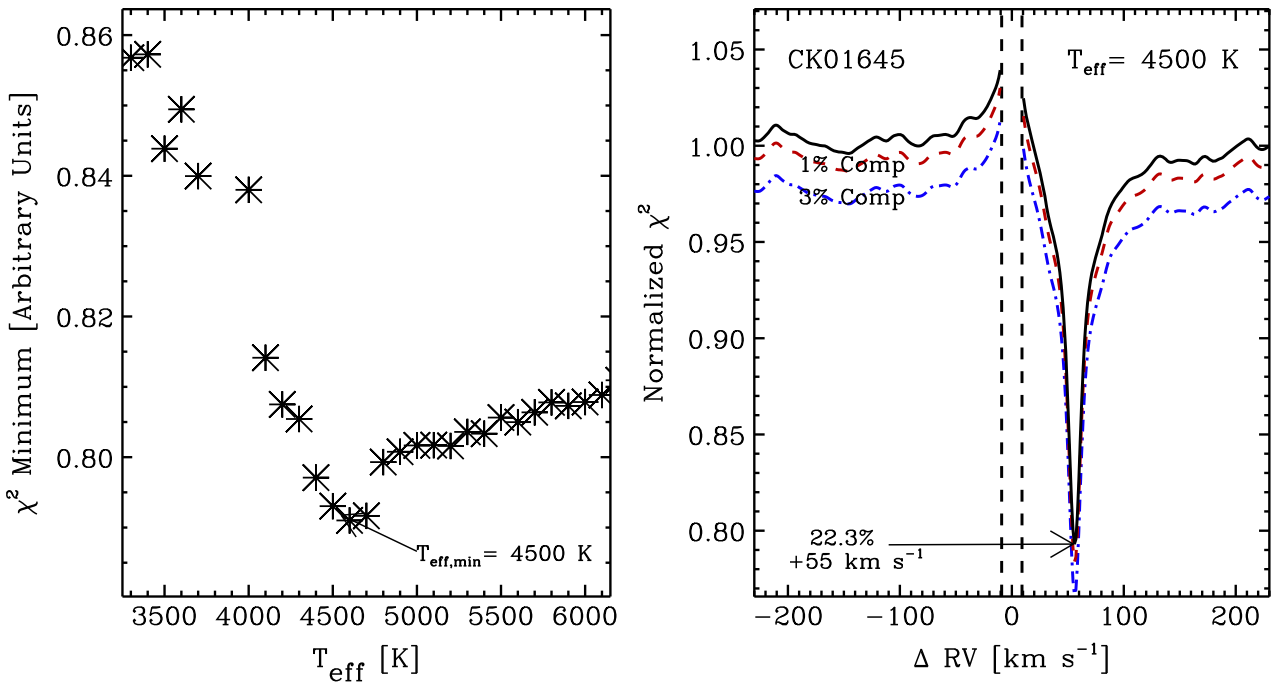

Figure A19. Final secondary star plot for KOI-1645. Same as Figure 9. 

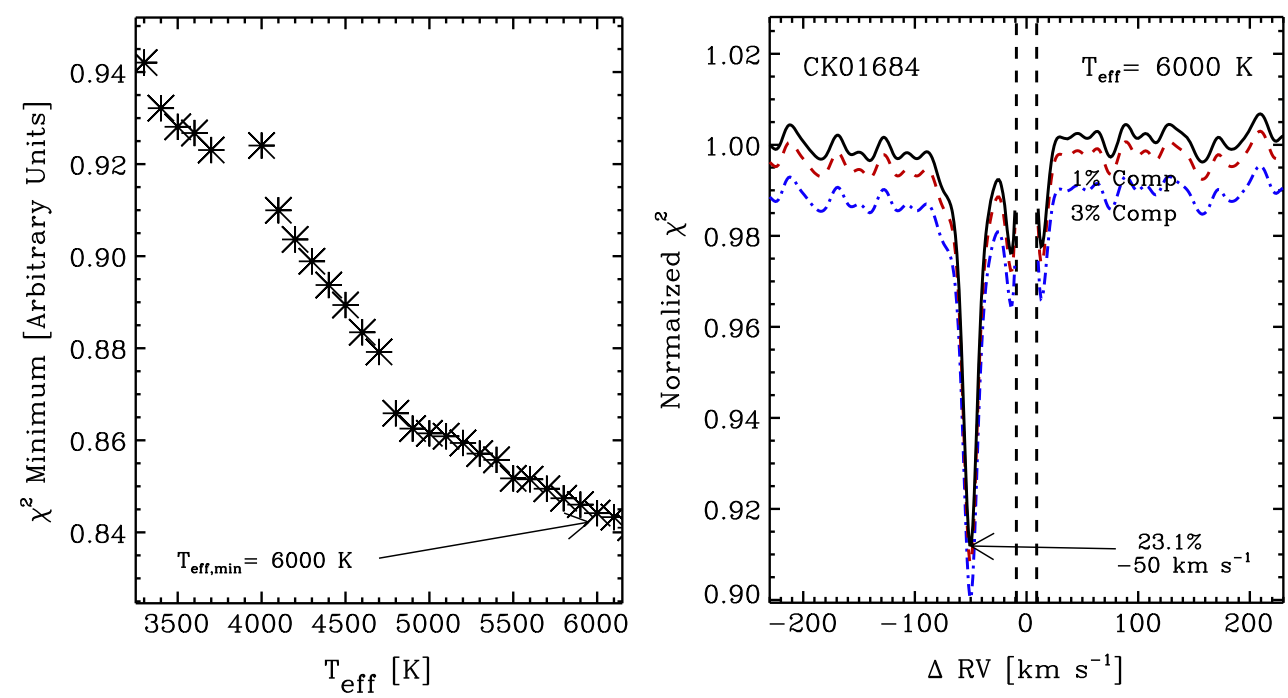

Figure A20. Final secondary star plot for KOI-1684. Same as Figure 9.
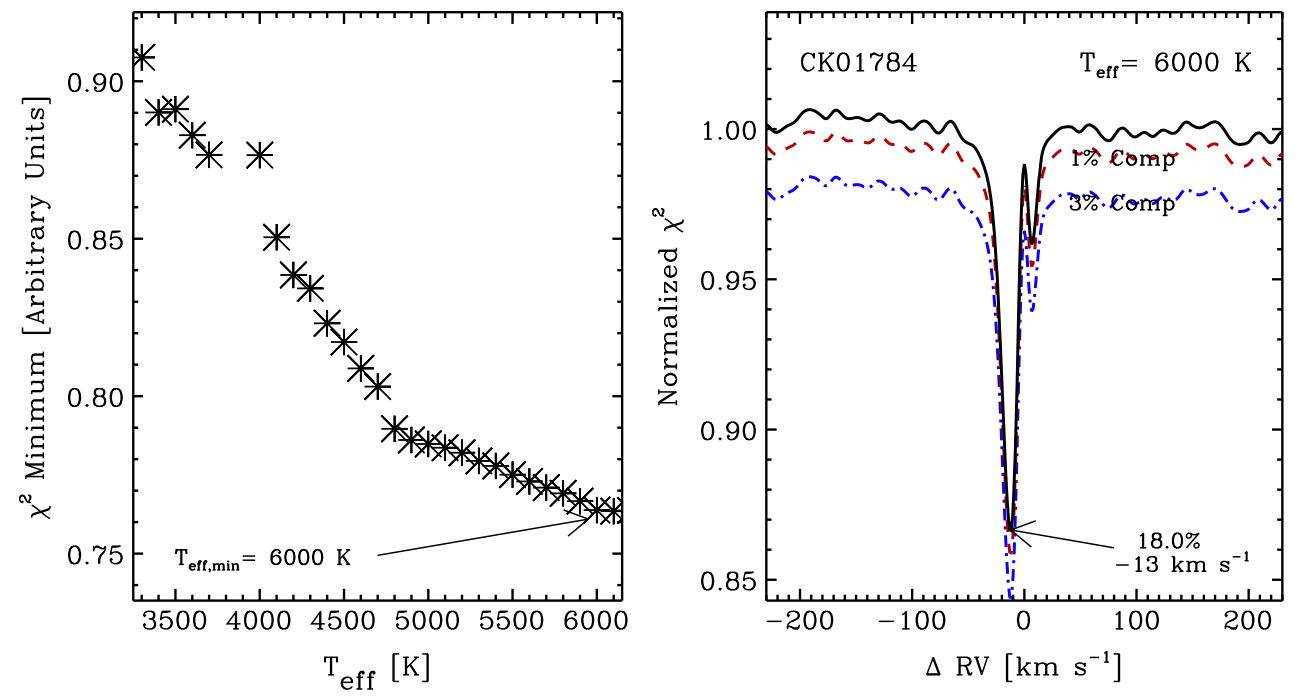

Figure A21. Final secondary star plot for KOI-1784. Same as Figure 9.
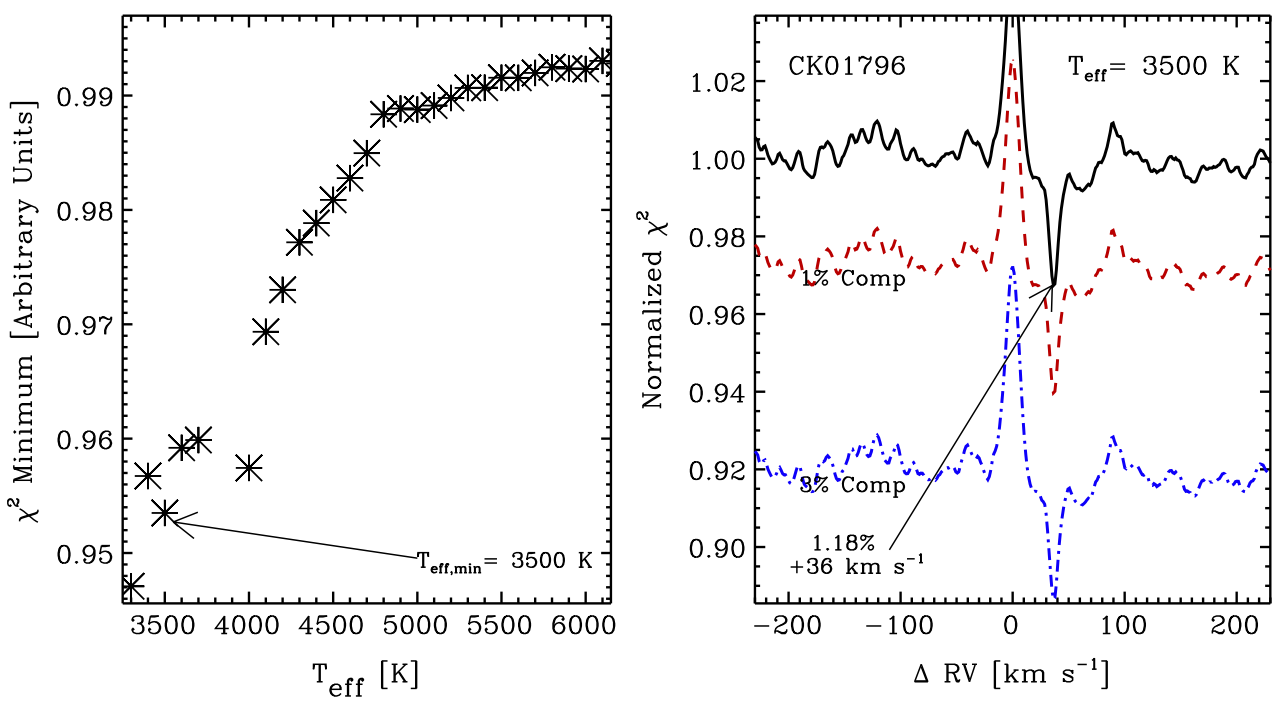

Figure A22. Final secondary star plot for KOI-1796. Same as Figure 9. 

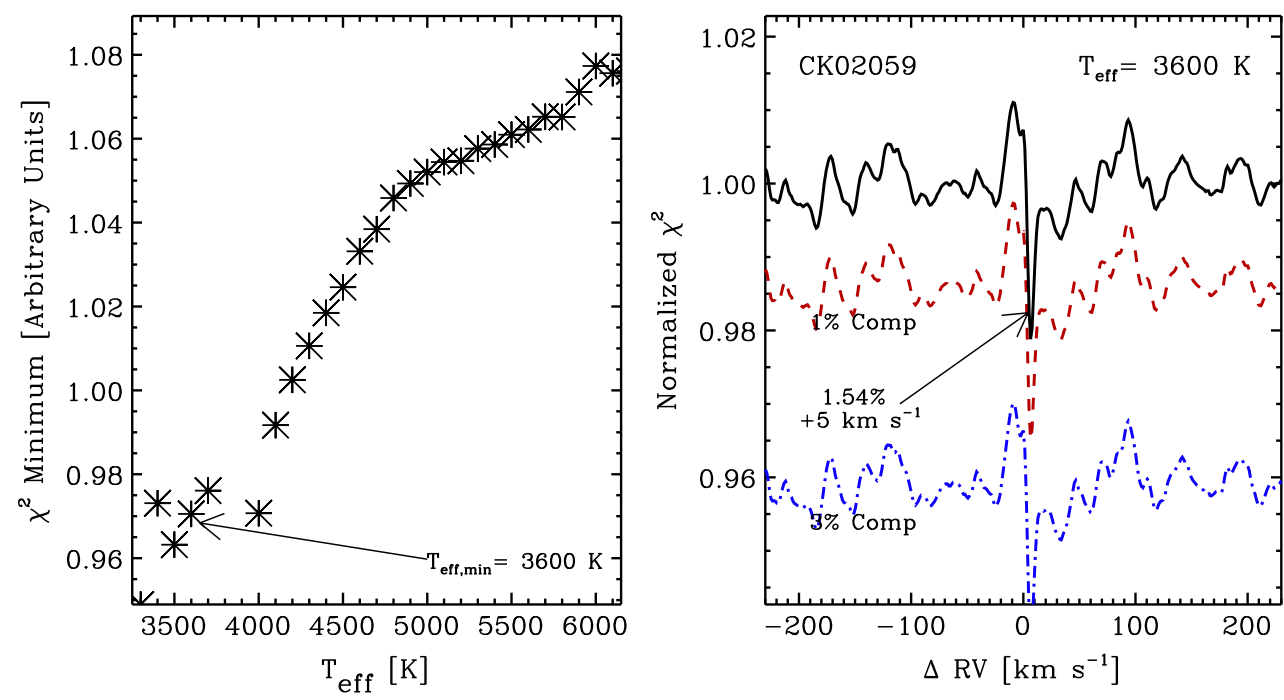

Figure A23. Final secondary star plot for KOI-2059. Same as Figure 9.
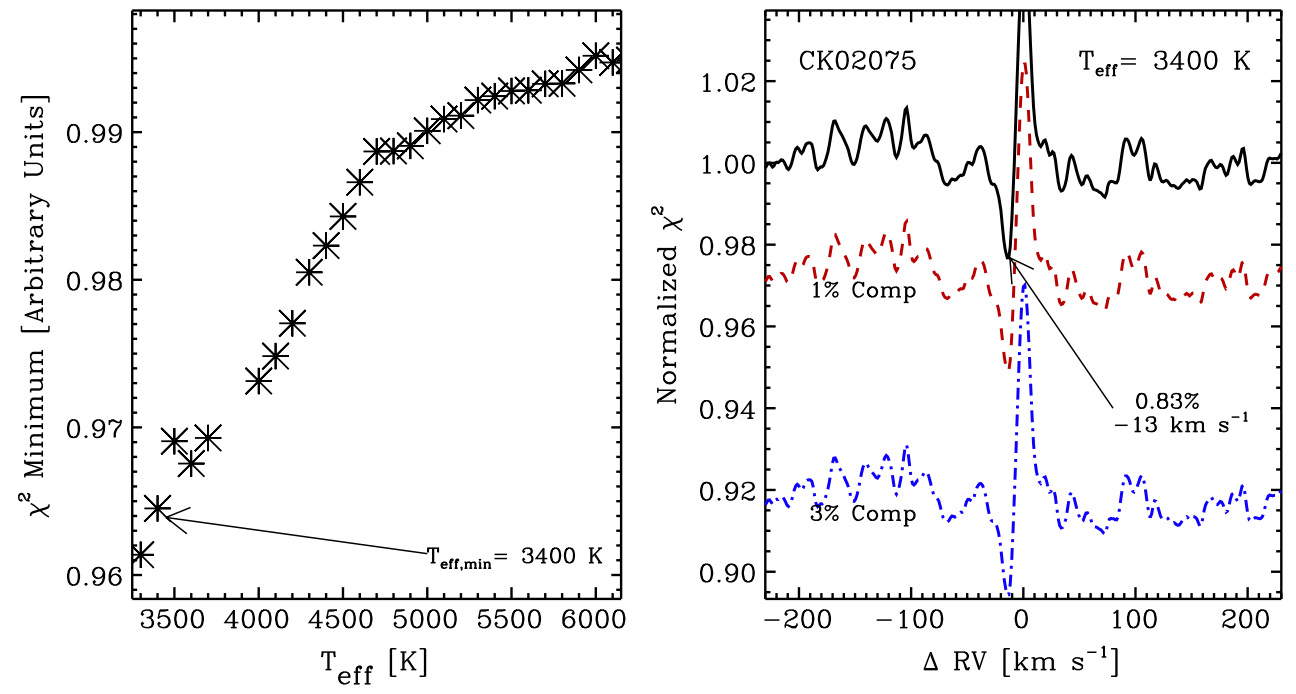

Figure A24. Final secondary star plot for KOI-2075. Same as Figure 9.
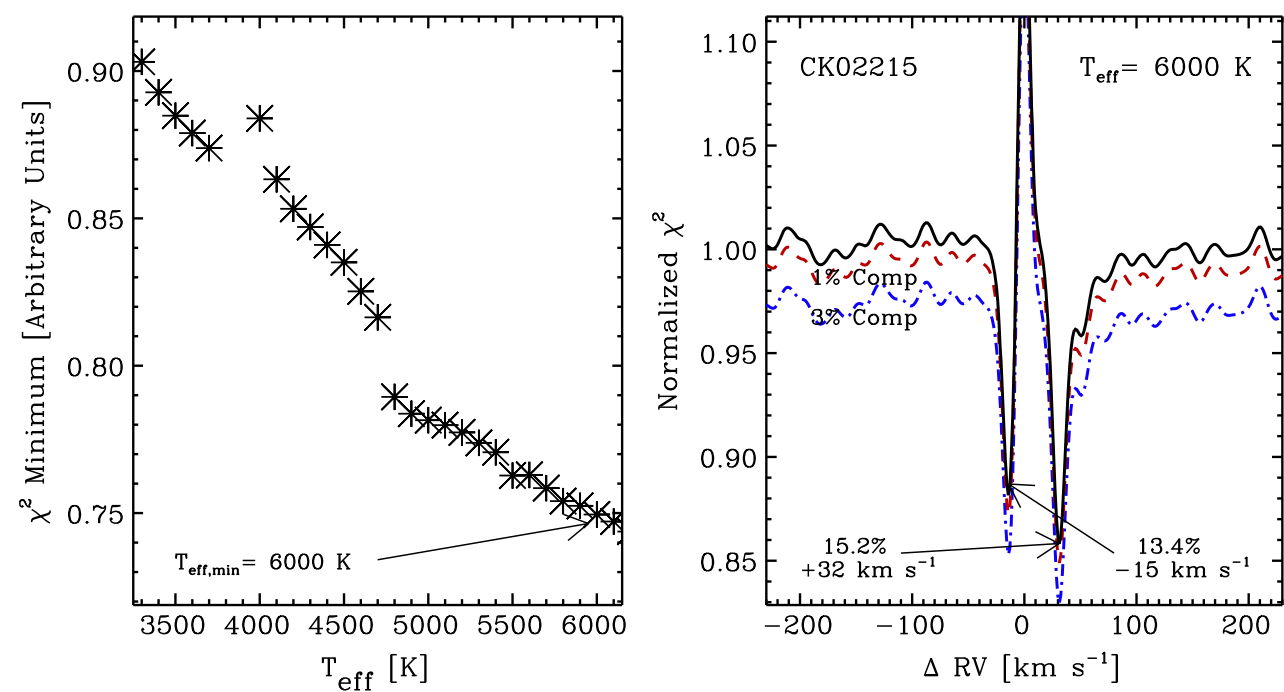

Figure A25. Final plot for KOI-2215. Same as Figure 9. Only the brightest secondary star $\chi^{2}$ minima function is shown at left, while both additional stars are annotated with an arrow at right. 

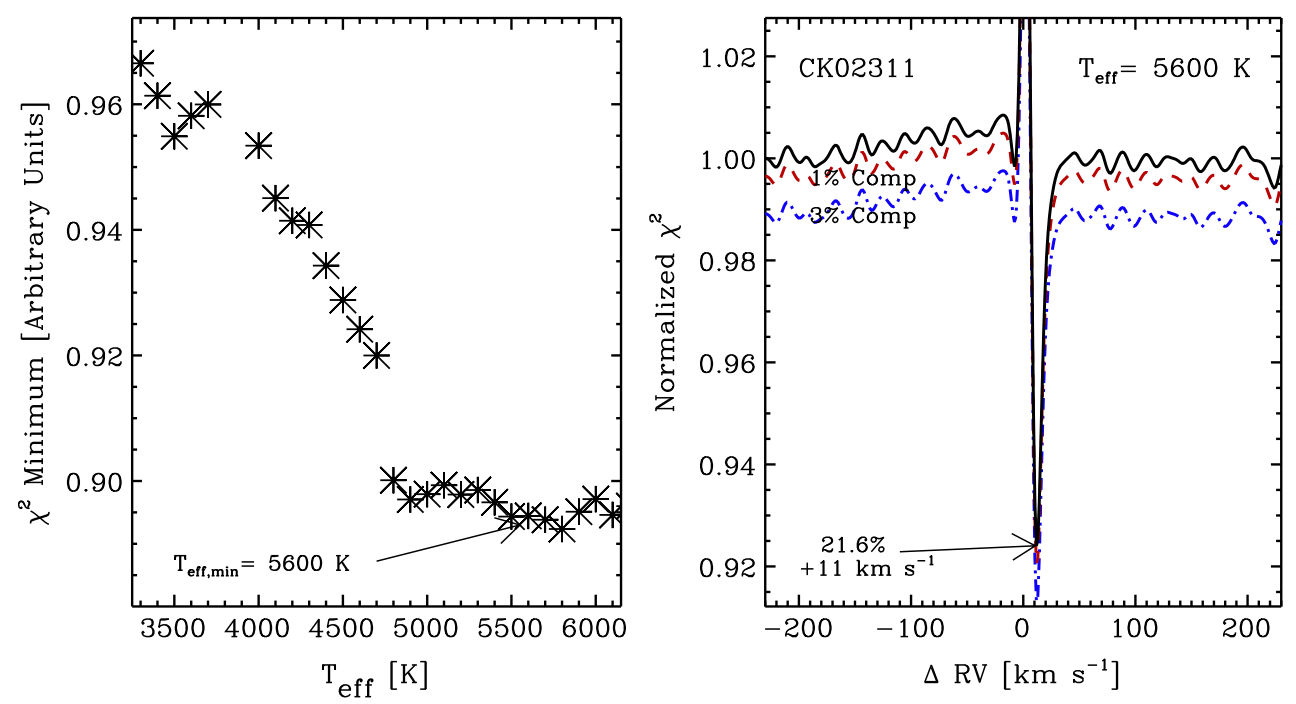

Figure A26. Final secondary star plot for KOI-2311. Same as Figure 9.
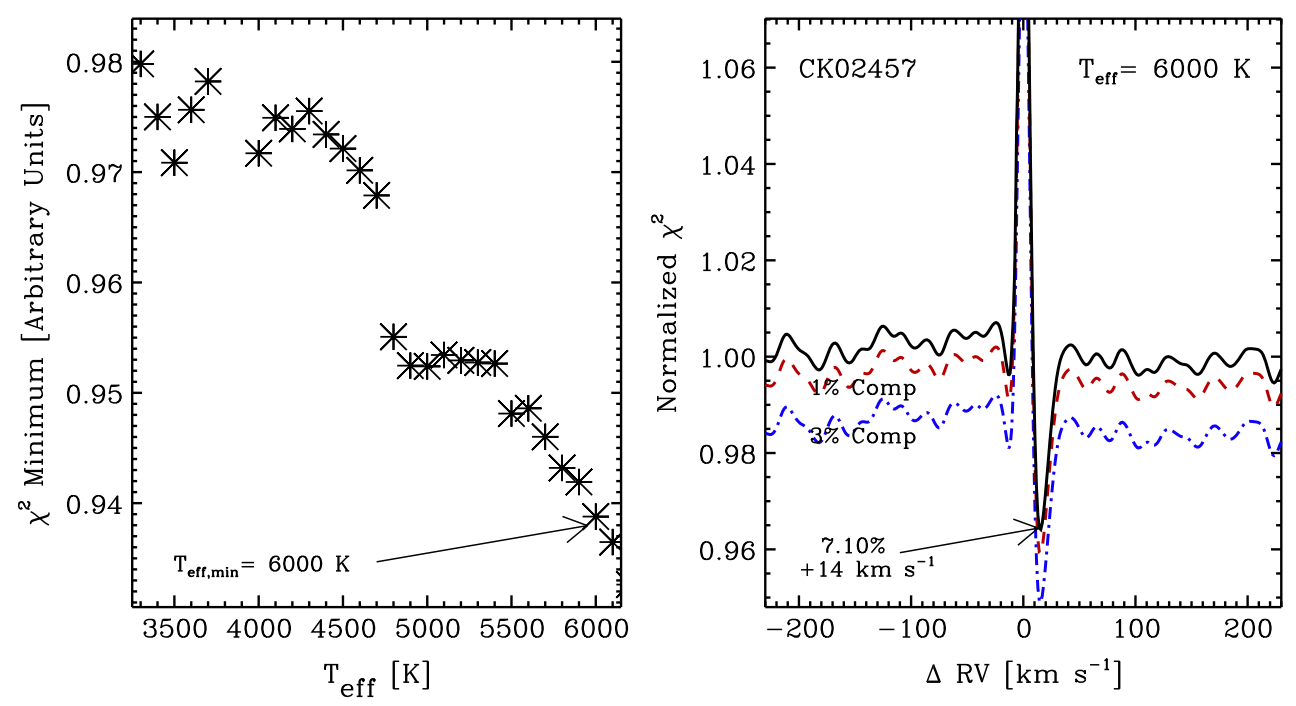

Figure A27. Final secondary star plot for KOI-2457. Same as Figure 9.
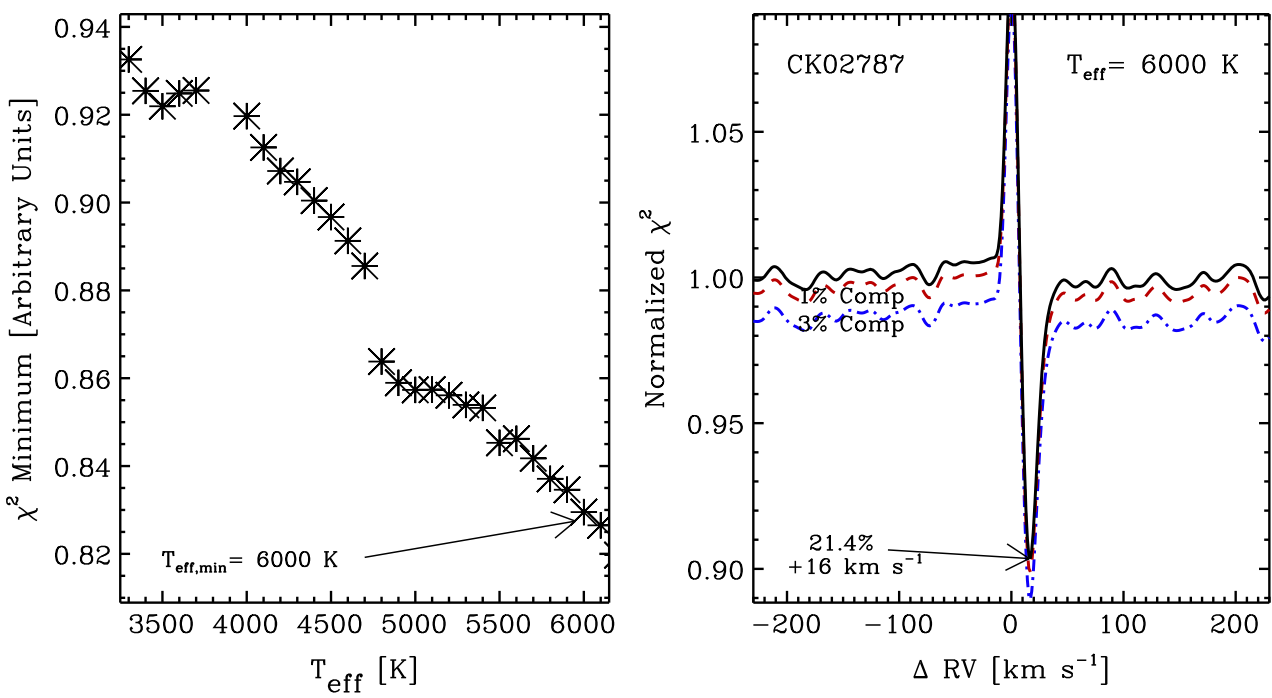

Figure A28. Final secondary star plot for KOI-2787. Same as Figure 9. 

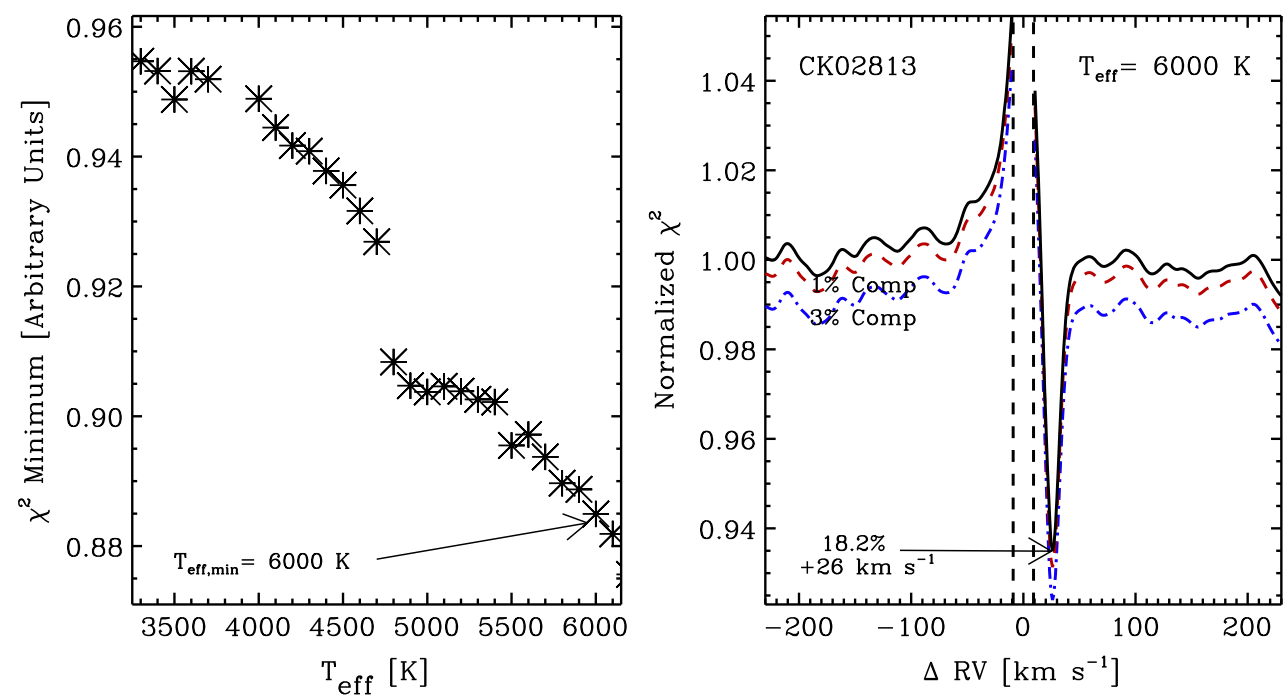

Figure A29. Final secondary star plot for KOI-2813. Same as Figure 9.
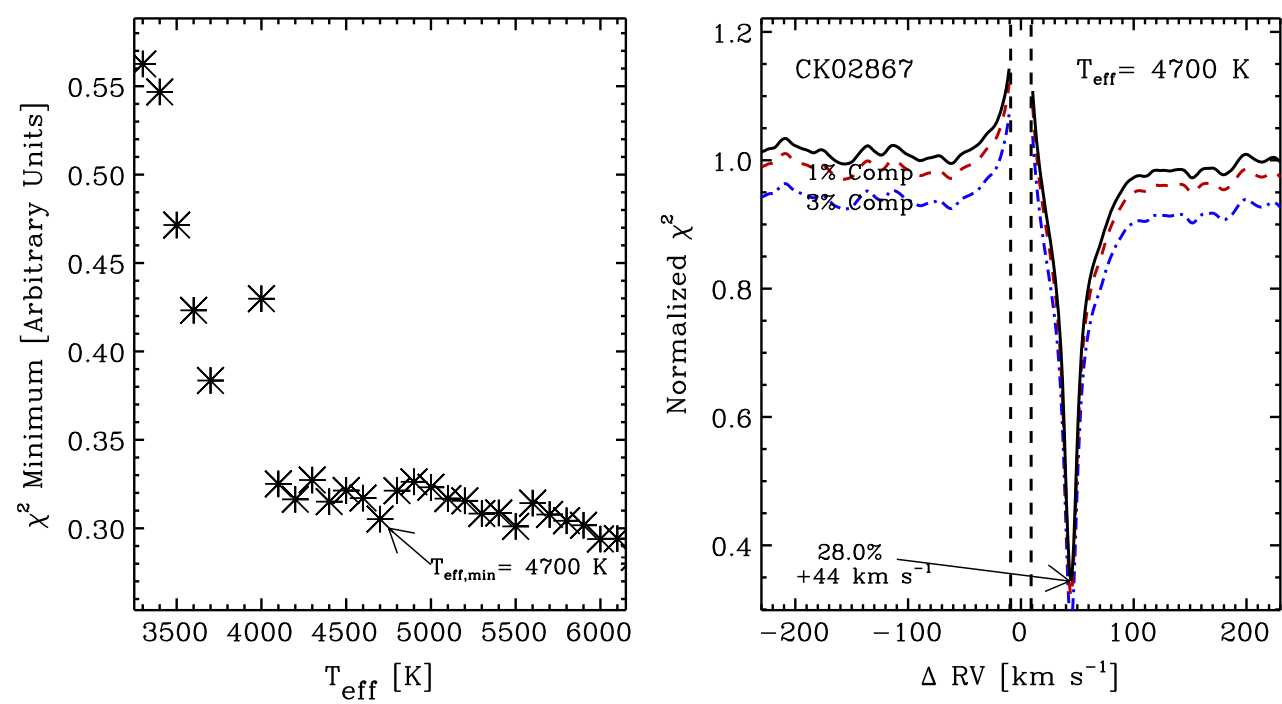

Figure A30. Final secondary star plot for KOI-2867. Same as Figure 9.
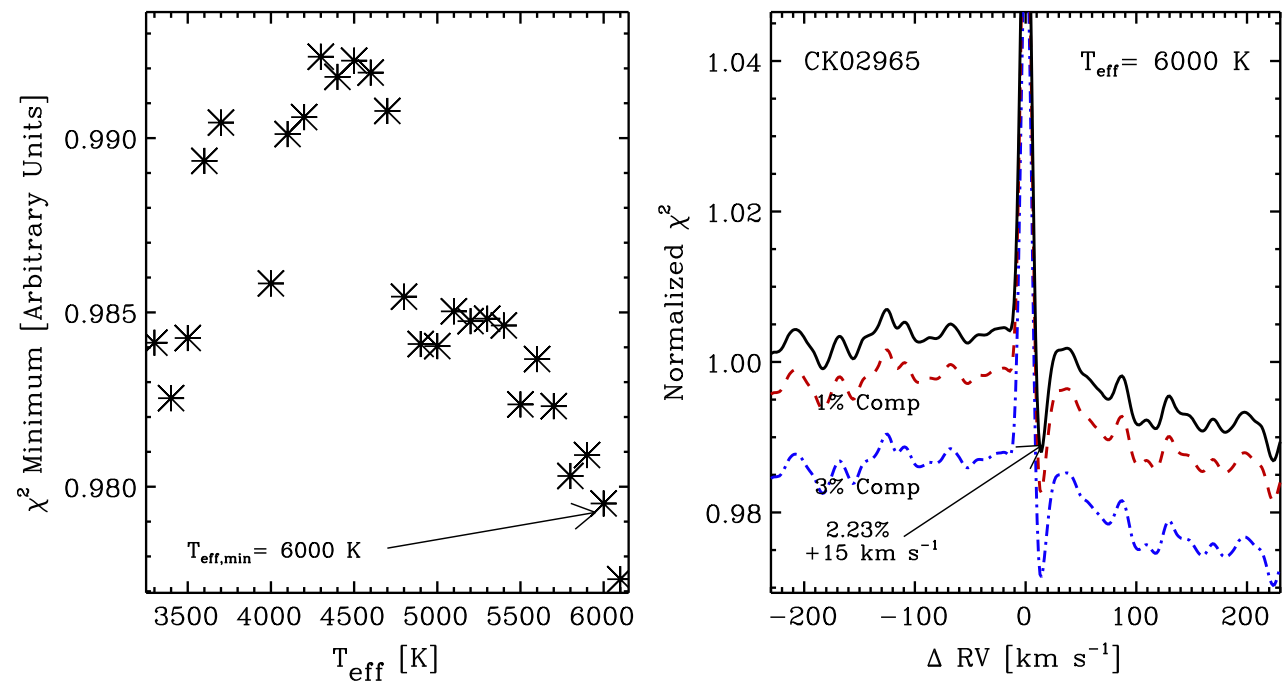

Figure A31. Final secondary star plot for KOI-2965. Same as Figure 9. 

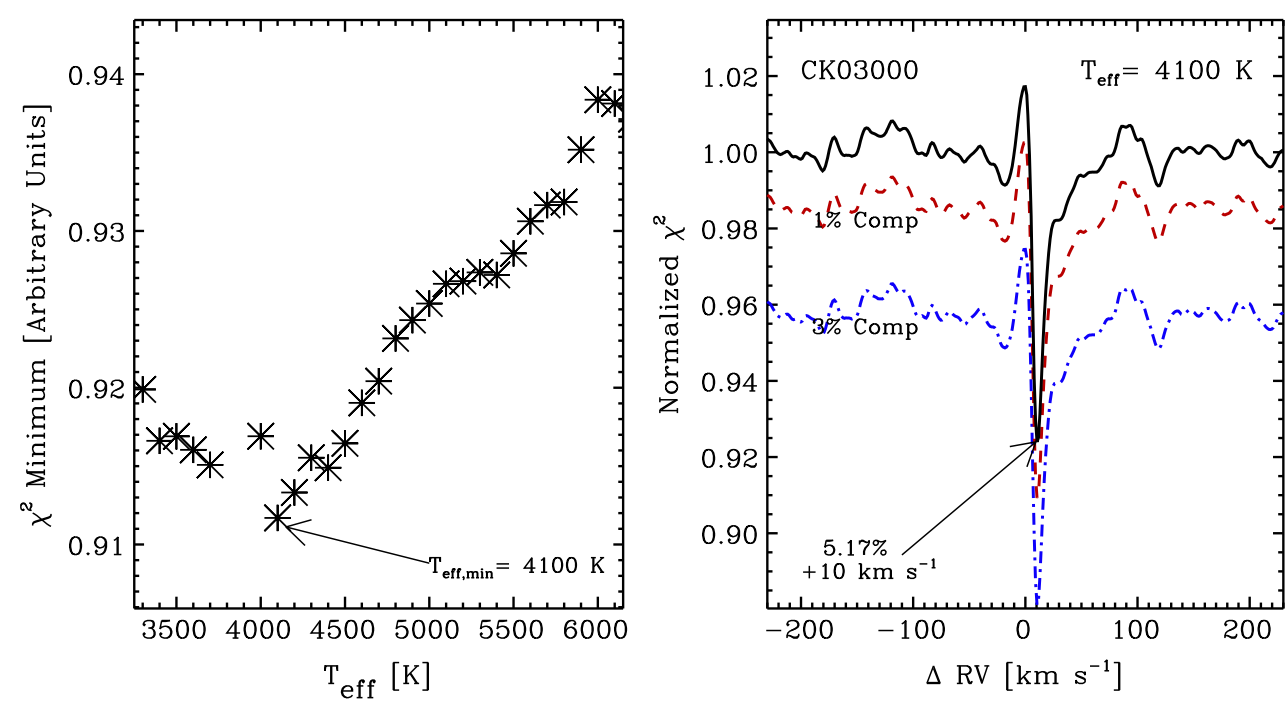

Figure A32. Final secondary star plot for KOI-3000. Same as Figure 9.
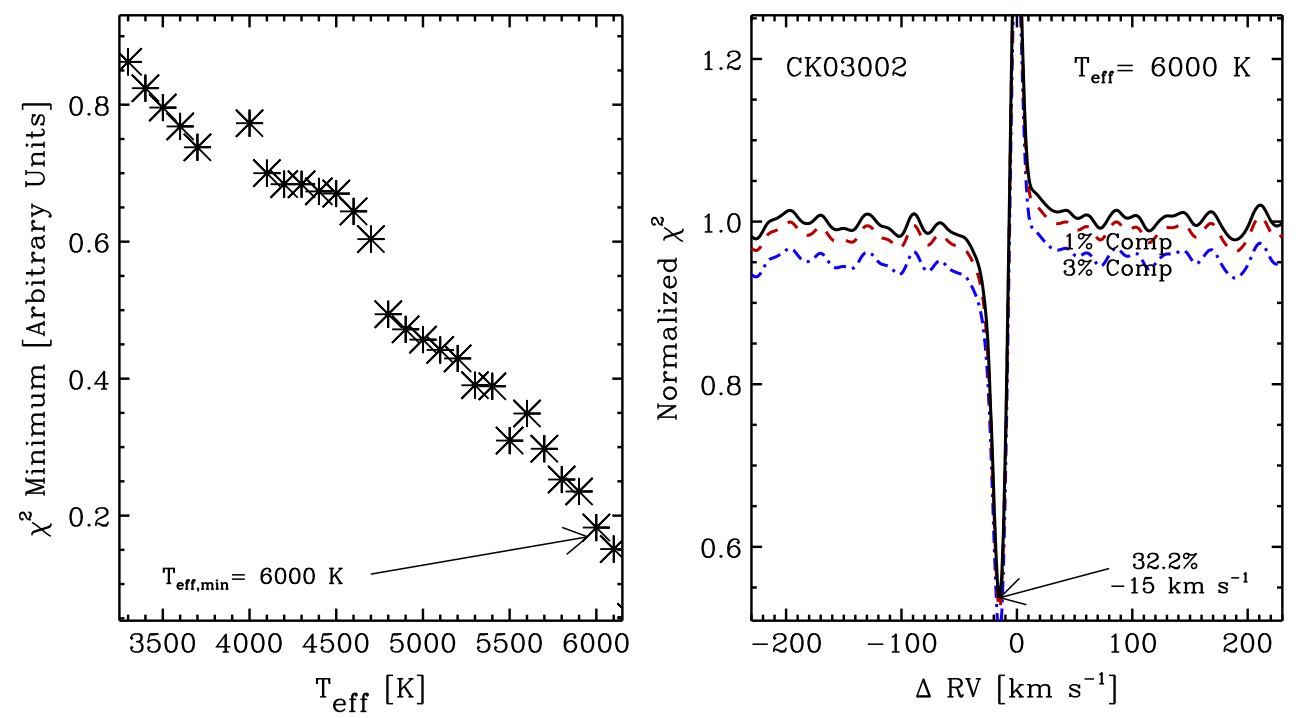

Figure A33. Final secondary star plot for KOI-3002. Same as Figure 9.
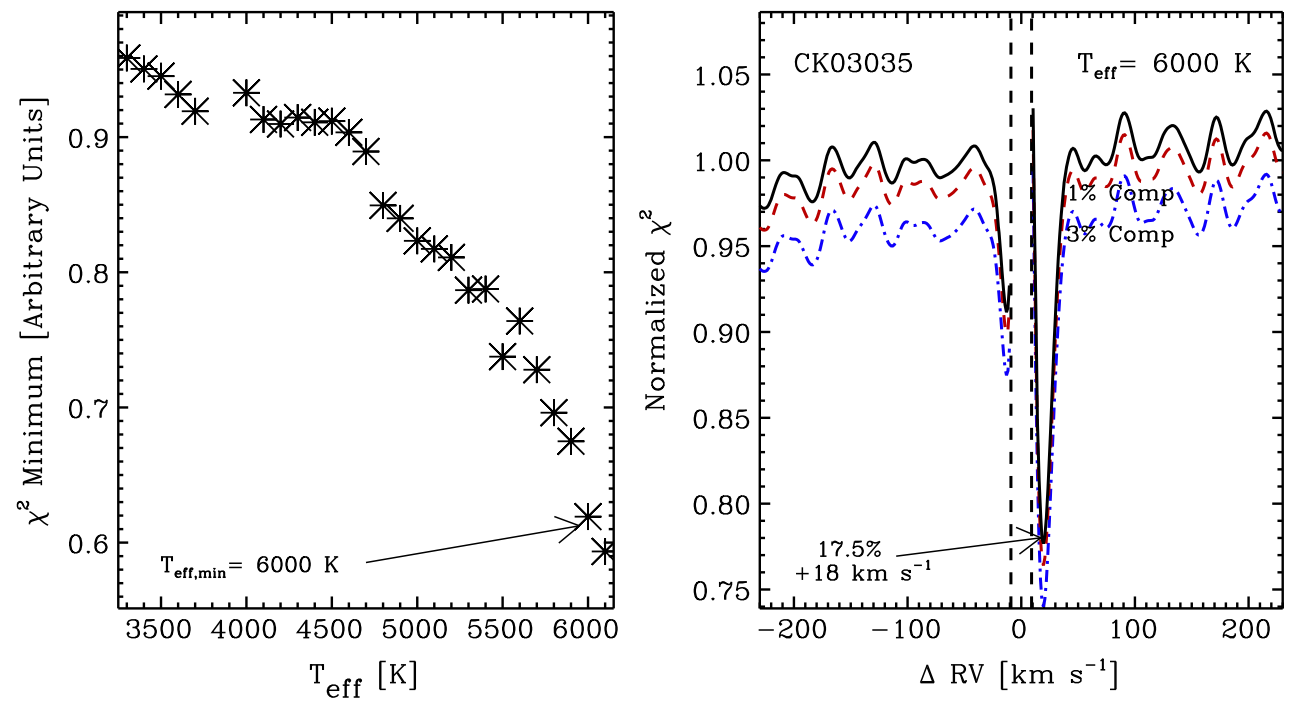

Figure A34. Final secondary star plot for KOI-3035. Same as Figure 9. 

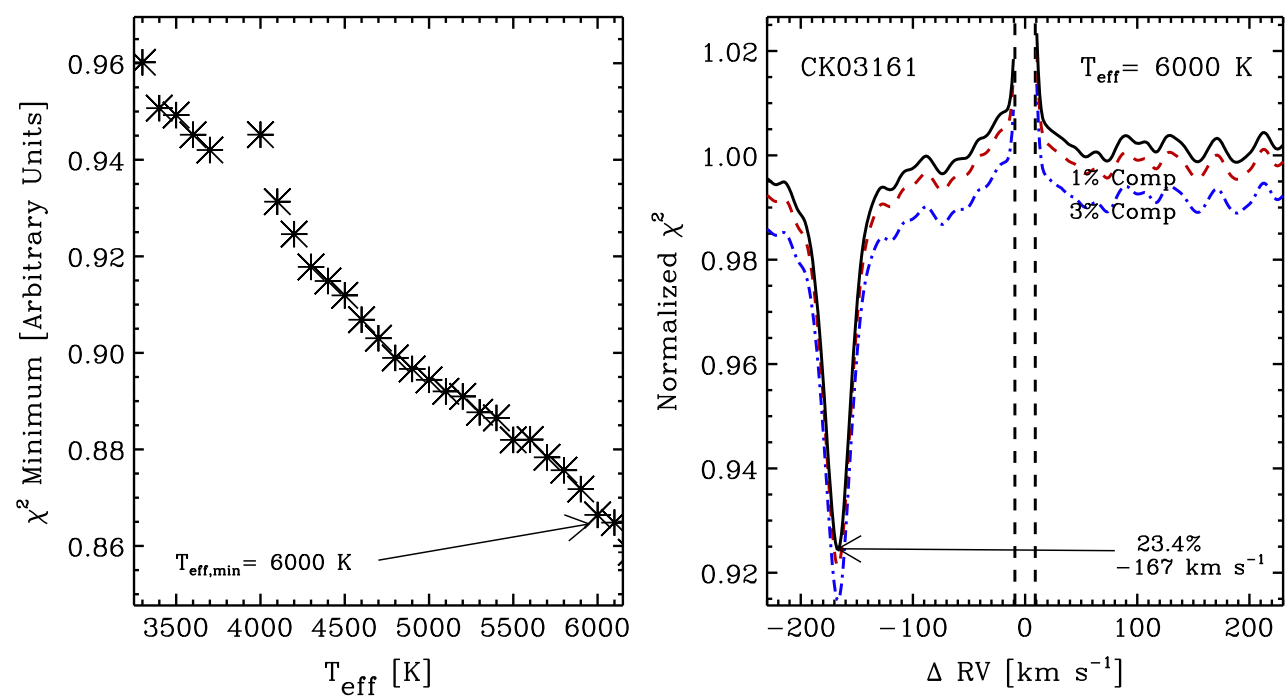

Figure A35. Final secondary star plot for KOI-3161. Same as Figure 9.
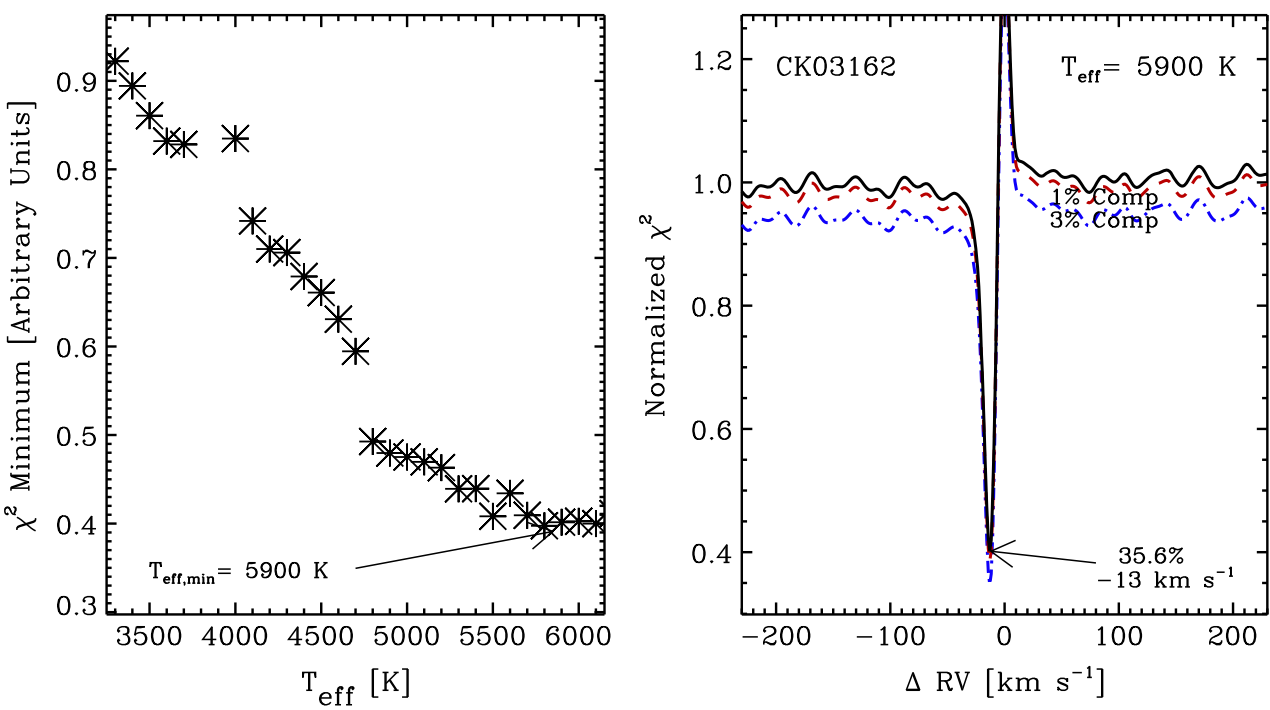

Figure A36. Final secondary star plot for KOI-3162. Same as Figure 9.
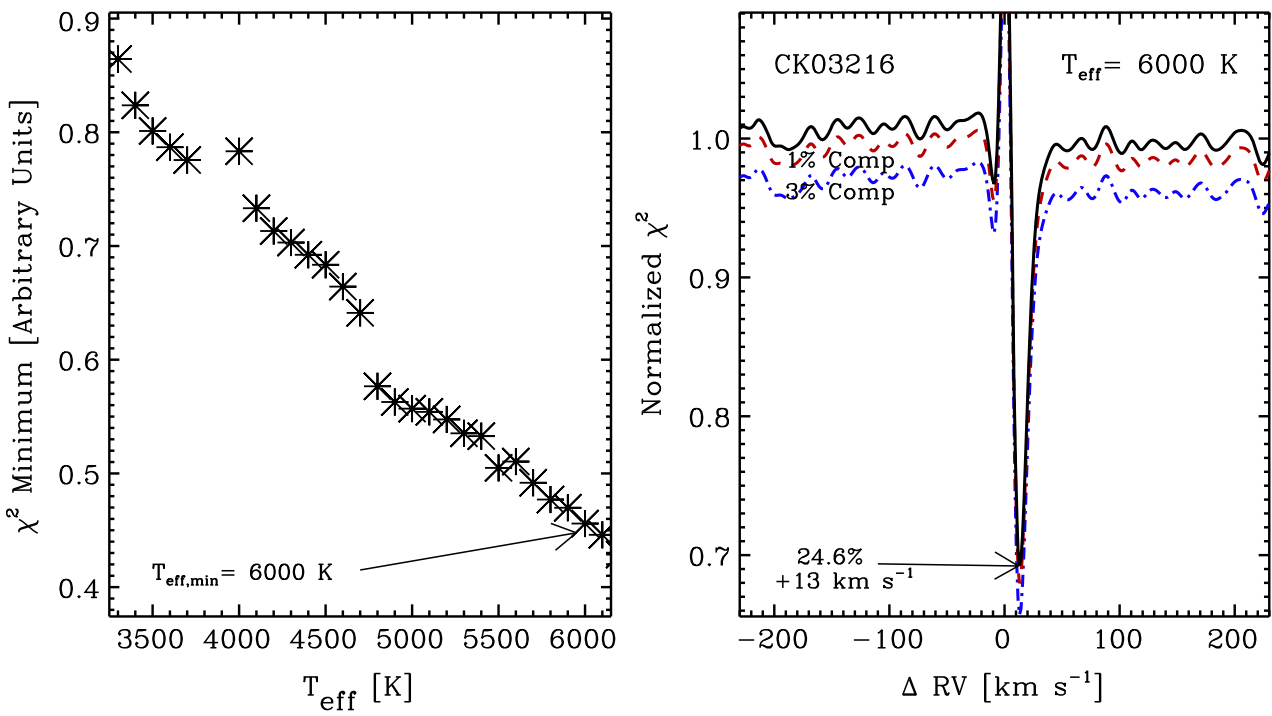

Figure A37. Final secondary star plot for KOI-3216. Same as Figure 9. 

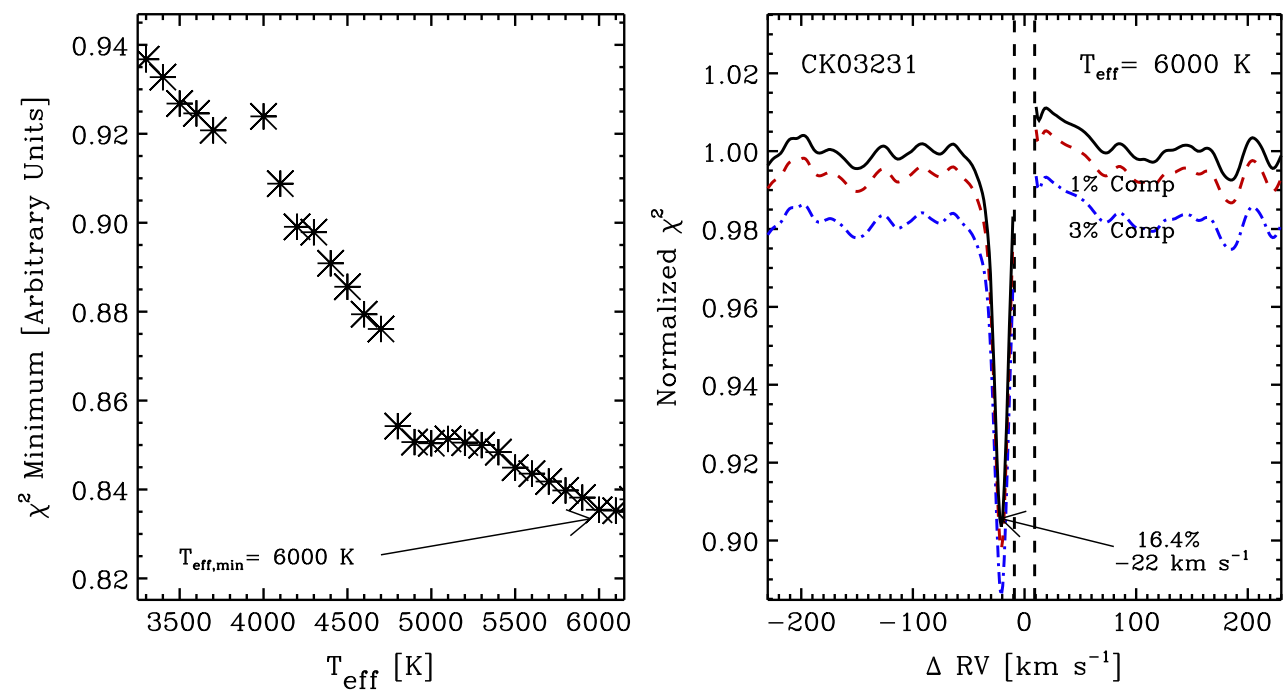

Figure A38. Final secondary star plot for KOI-3231. Same as Figure 9.
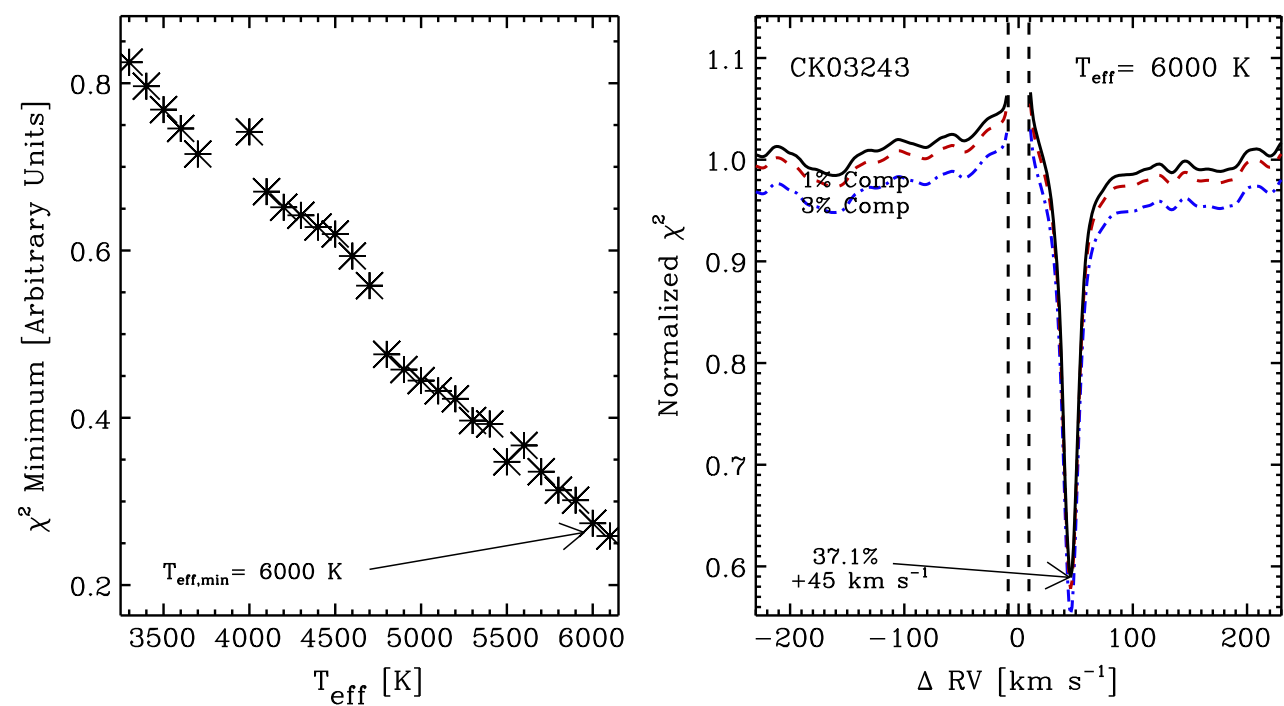

Figure A39. Final secondary star plot for KOI-3243. Same as Figure 9.
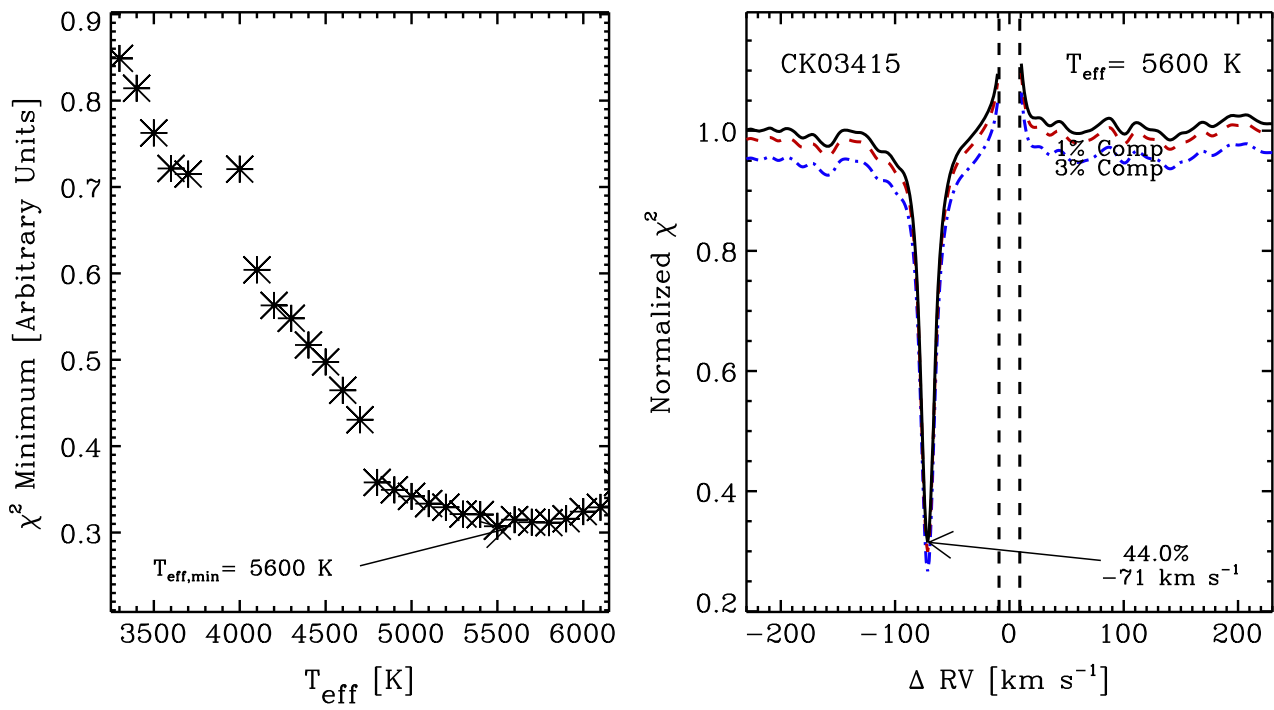

Figure A40. Final secondary star plot for KOI-3415. Same as Figure 9. 

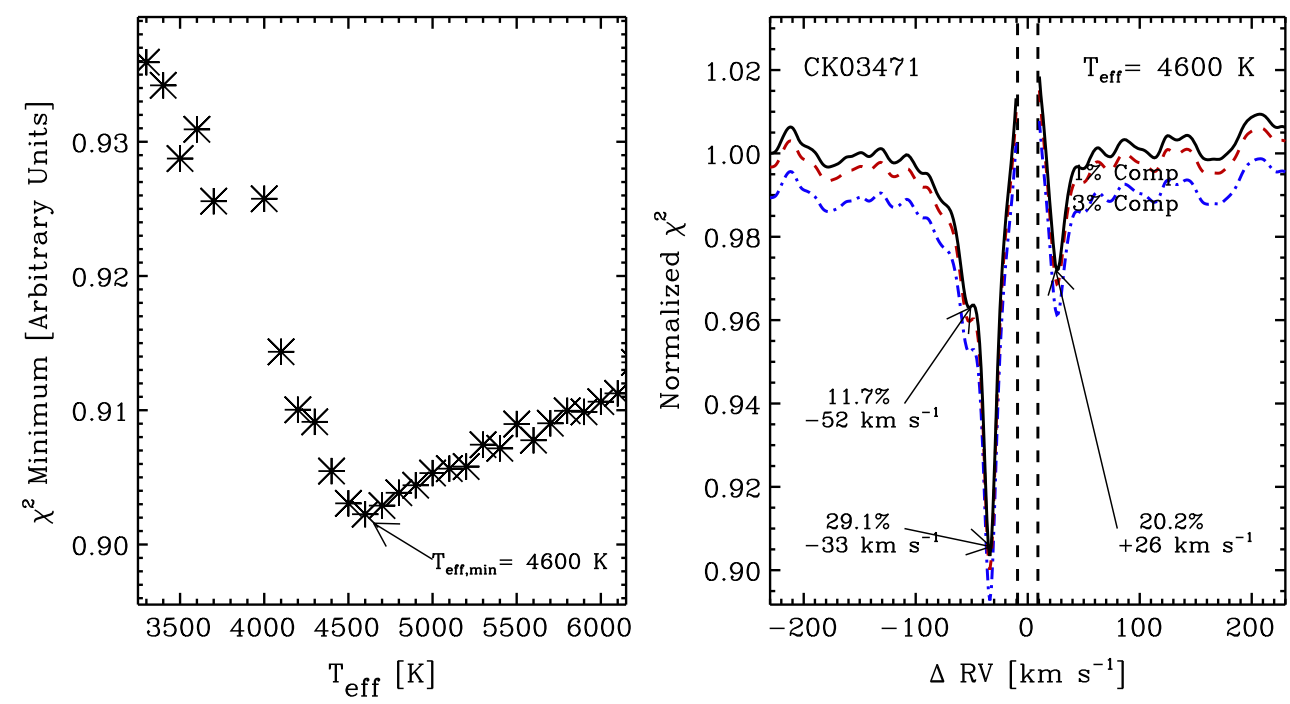

Figure A41. Final plot for KOI-3471. Same as Figure 9. At left, we show only the $\chi^{2}$ minima function for the brightest secondary star. At left, we annotate all three detected additional stars to KOI-3471 with an arrow.
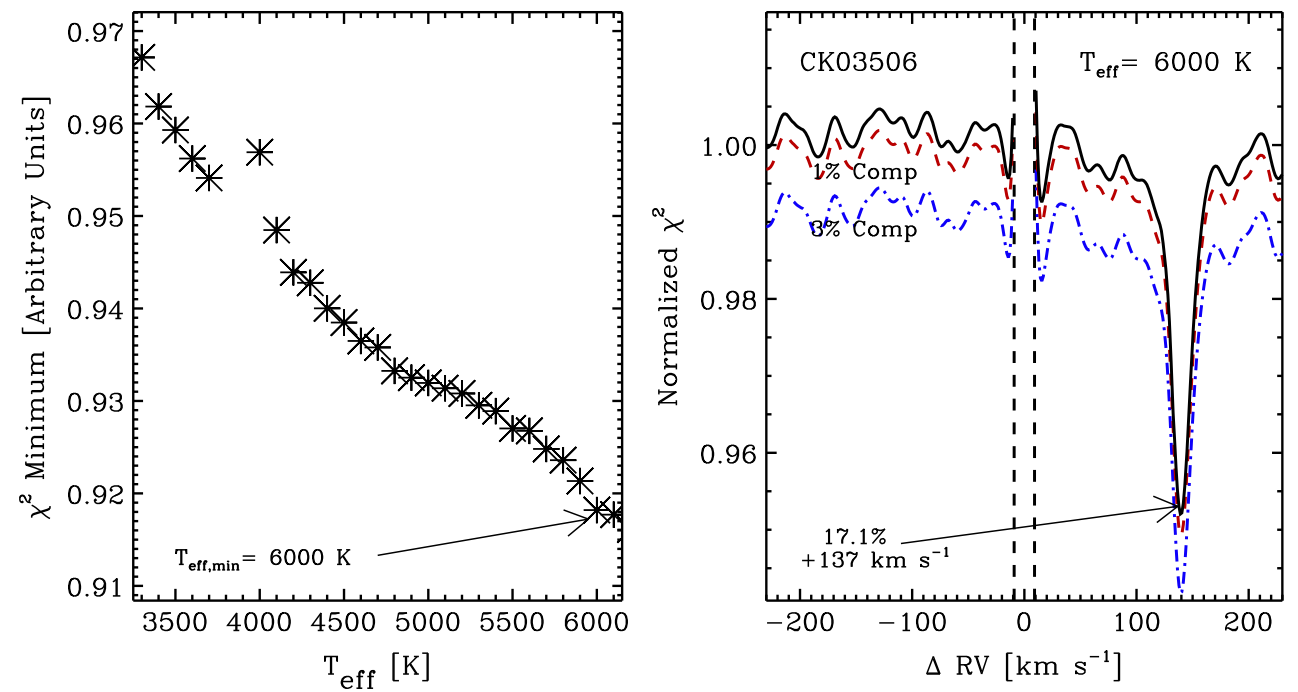

Figure A42. Final secondary star plot for KOI-3506. Same as Figure 9.
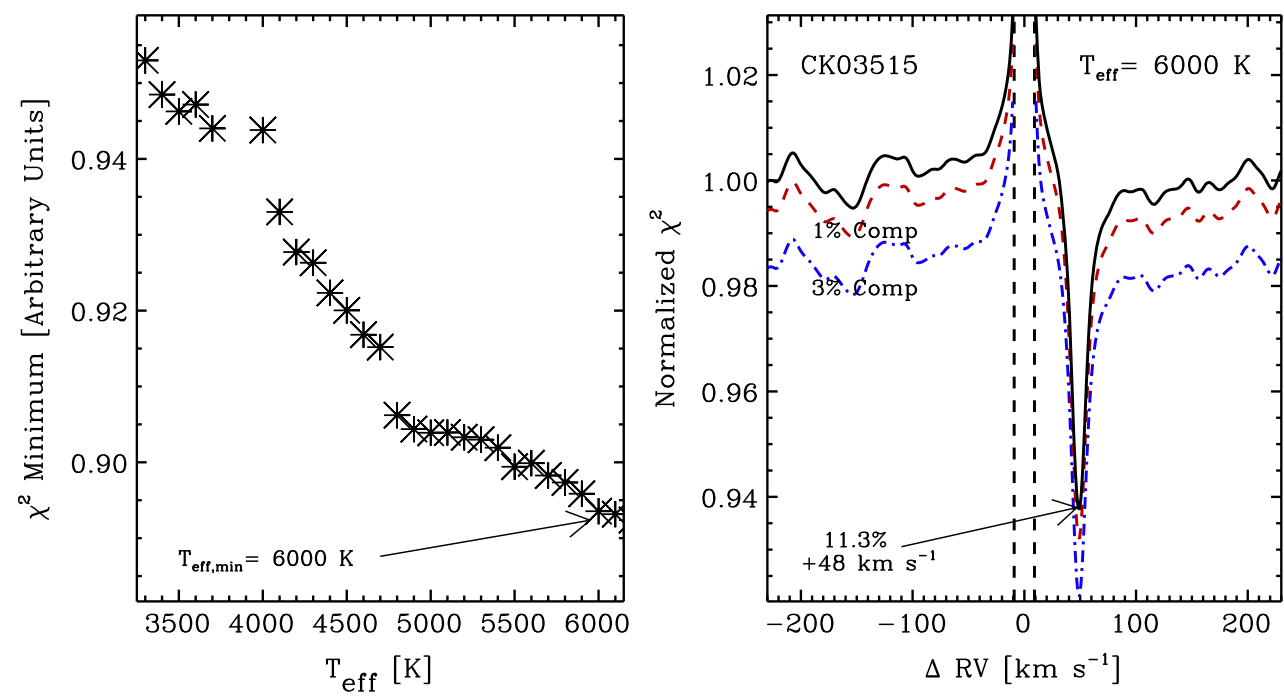

Figure A43. Final secondary star plot for KOI-3515. Same as Figure 9. 

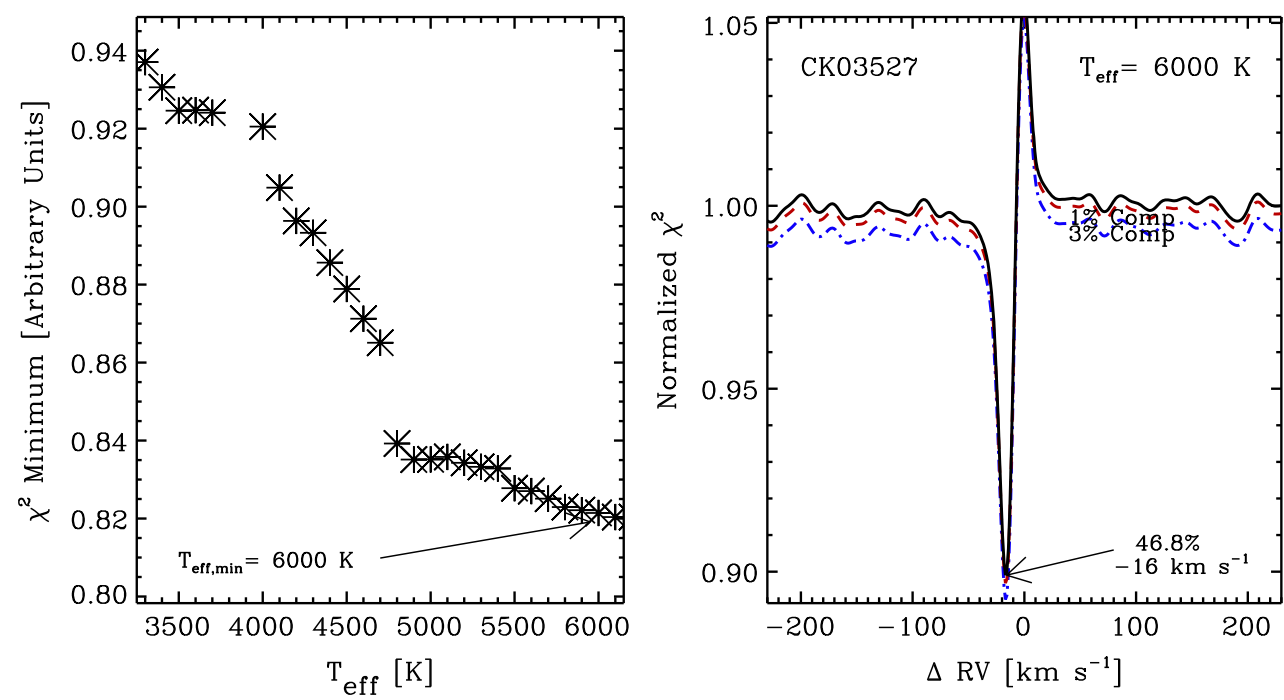

Figure A44. Final secondary star plot for KOI-3527. Same as Figure 9.
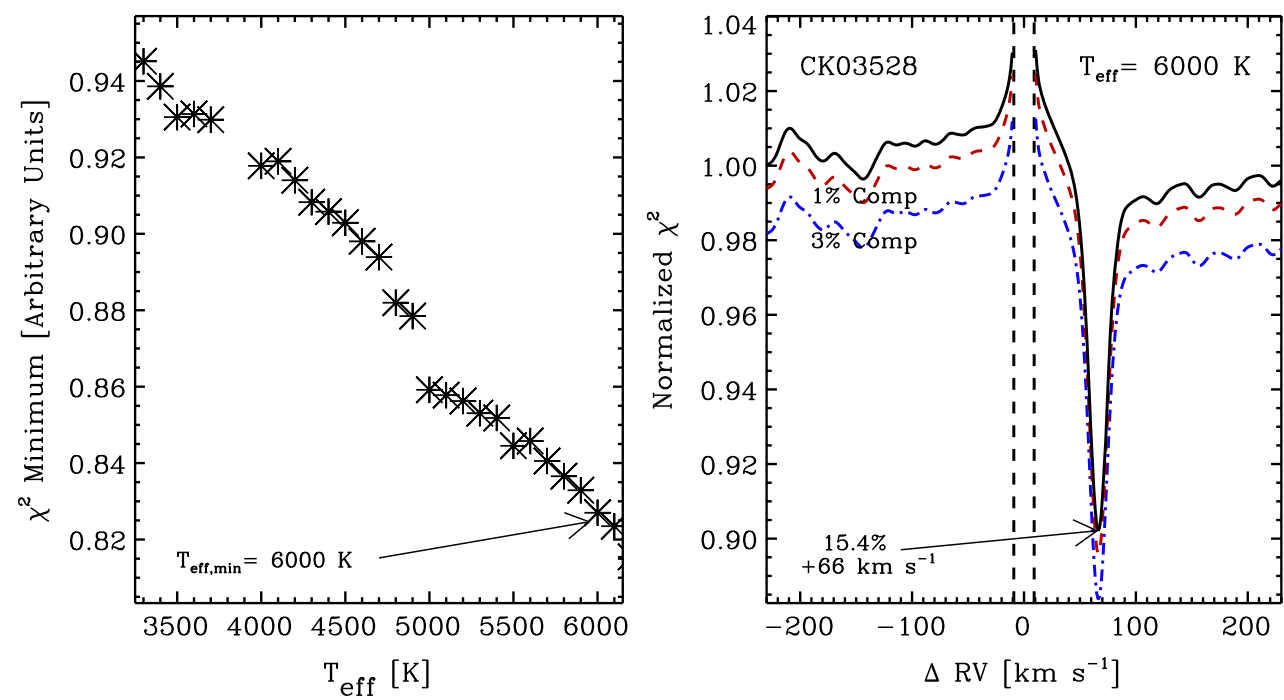

Figure A45. Final secondary star plot for KOI-3528. Same as Figure 9.
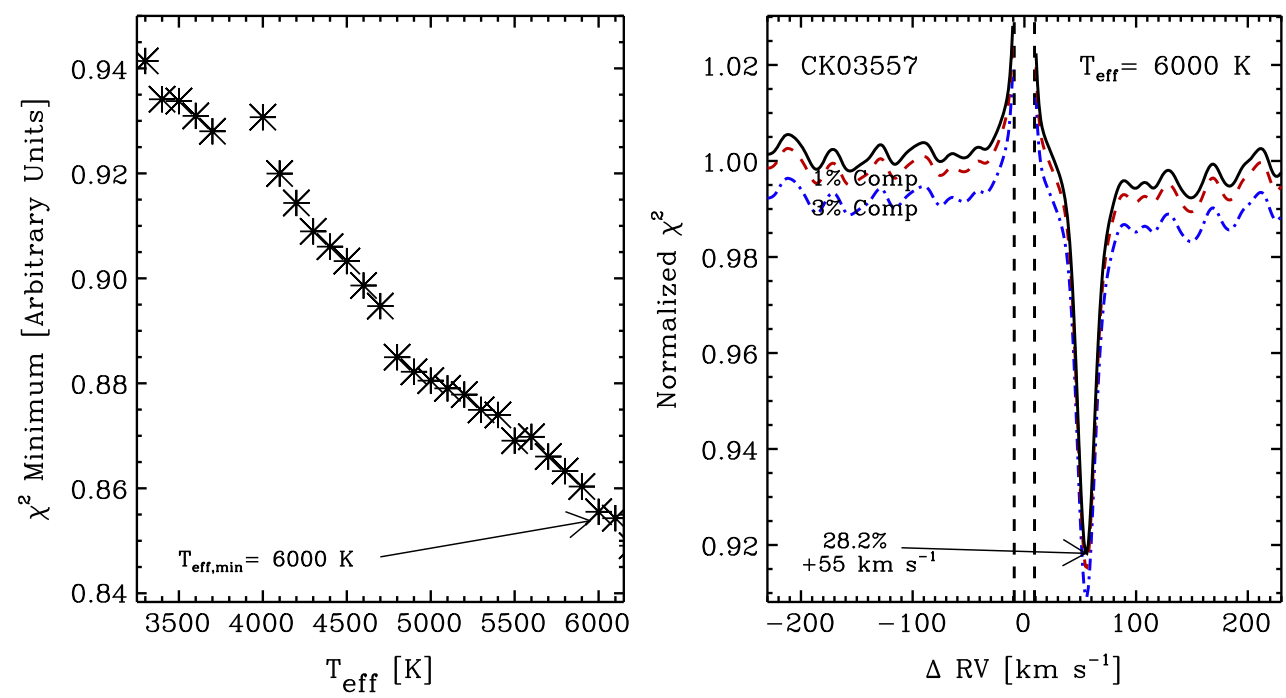

Figure A46. Final secondary star plot for KOI-3557. Same as Figure 9. 

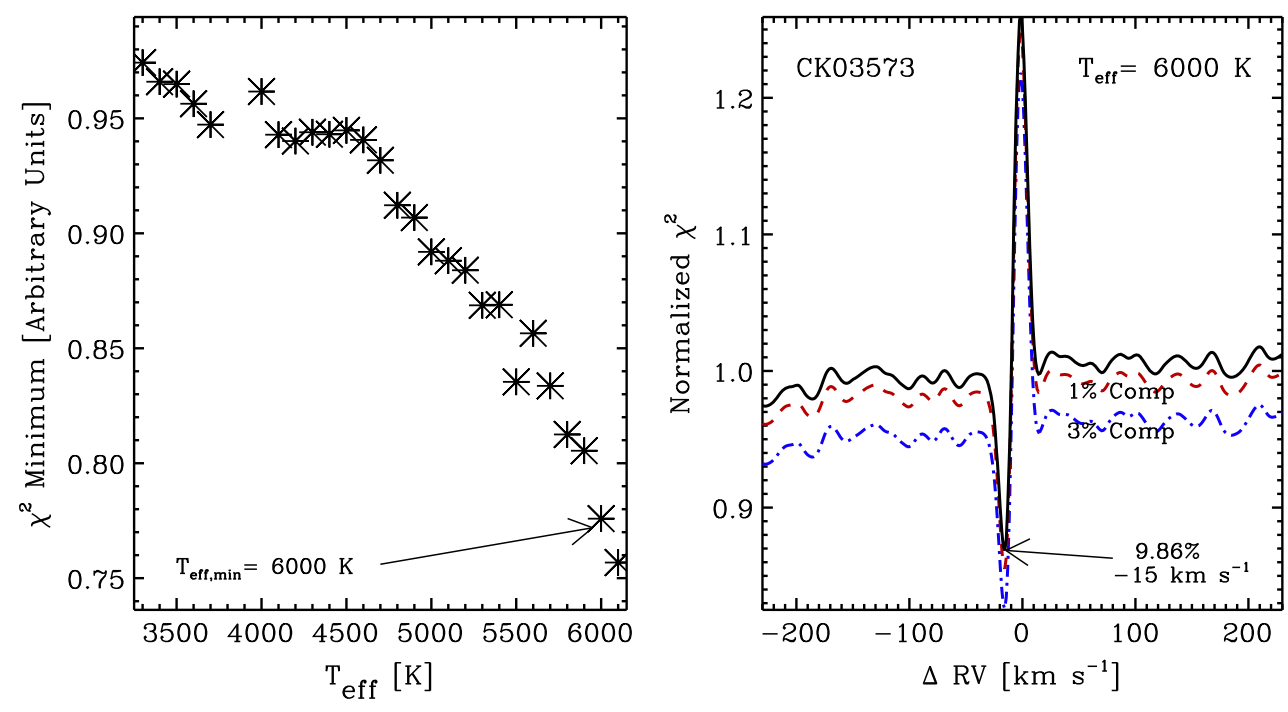

Figure A47. Final secondary star plot for KOI-3573. Same as Figure 9.
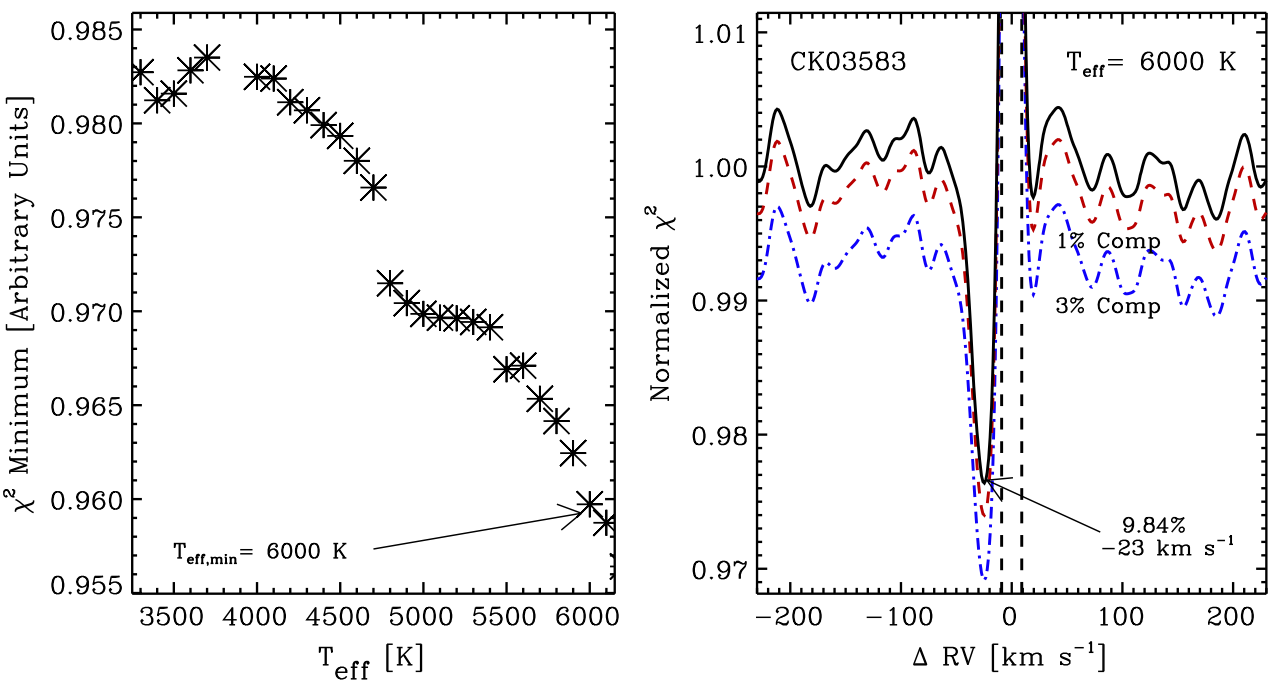

Figure A48. Final secondary star plot for KOI-3583. Same as Figure 9.
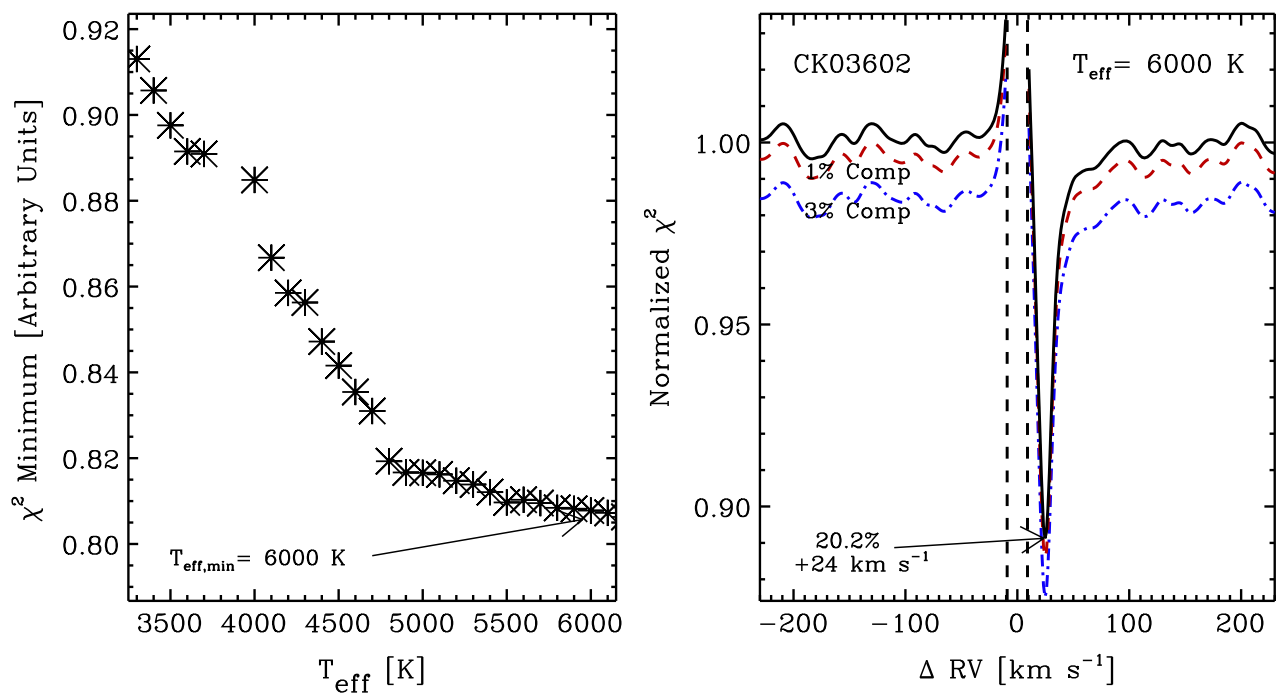

Figure A49. Final secondary star plot for KOI-3602. Same as Figure 9. 

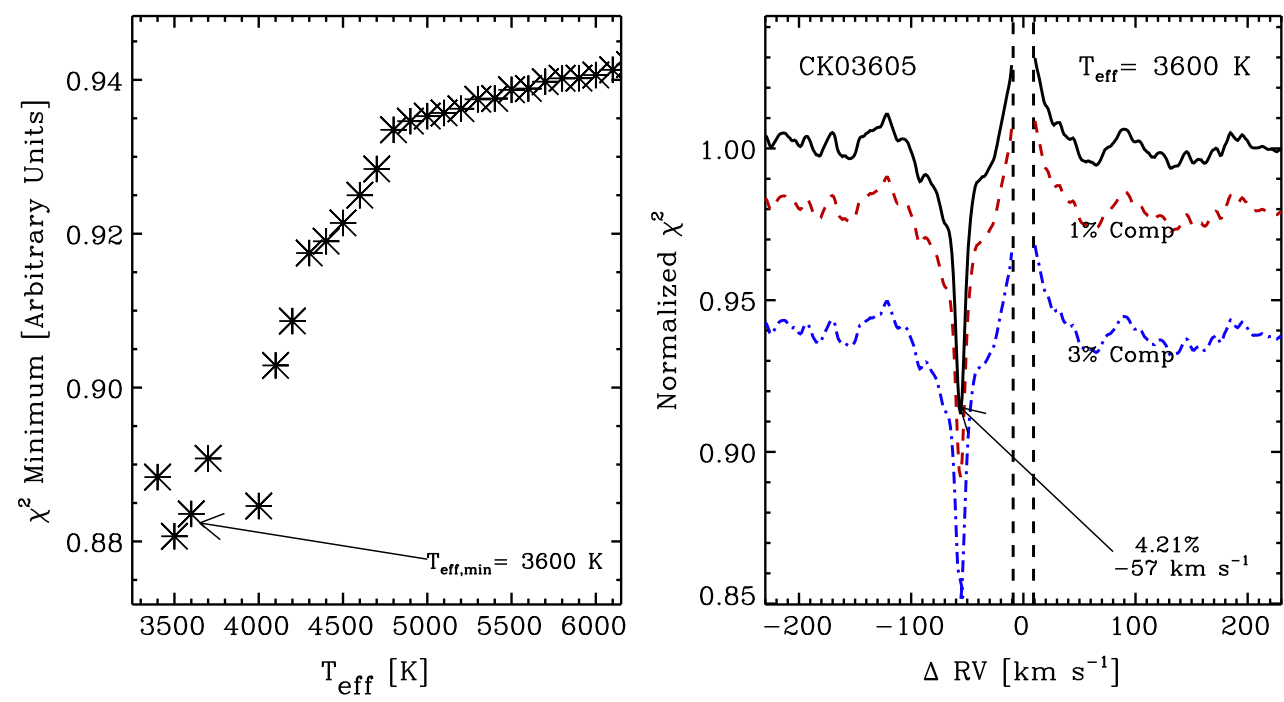

Figure A50. Final secondary star plot for KOI-3605. Same as Figure 9.
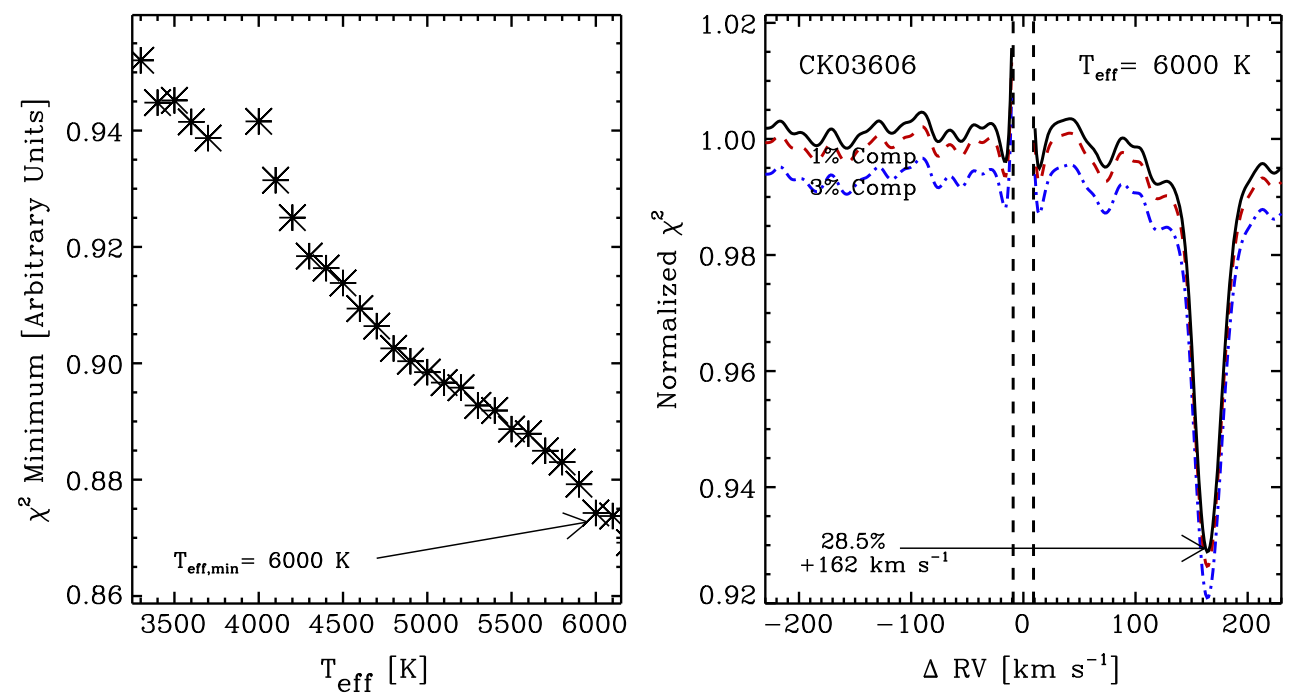

Figure A51. Final secondary star plot for KOI-3606. Same as Figure 9.
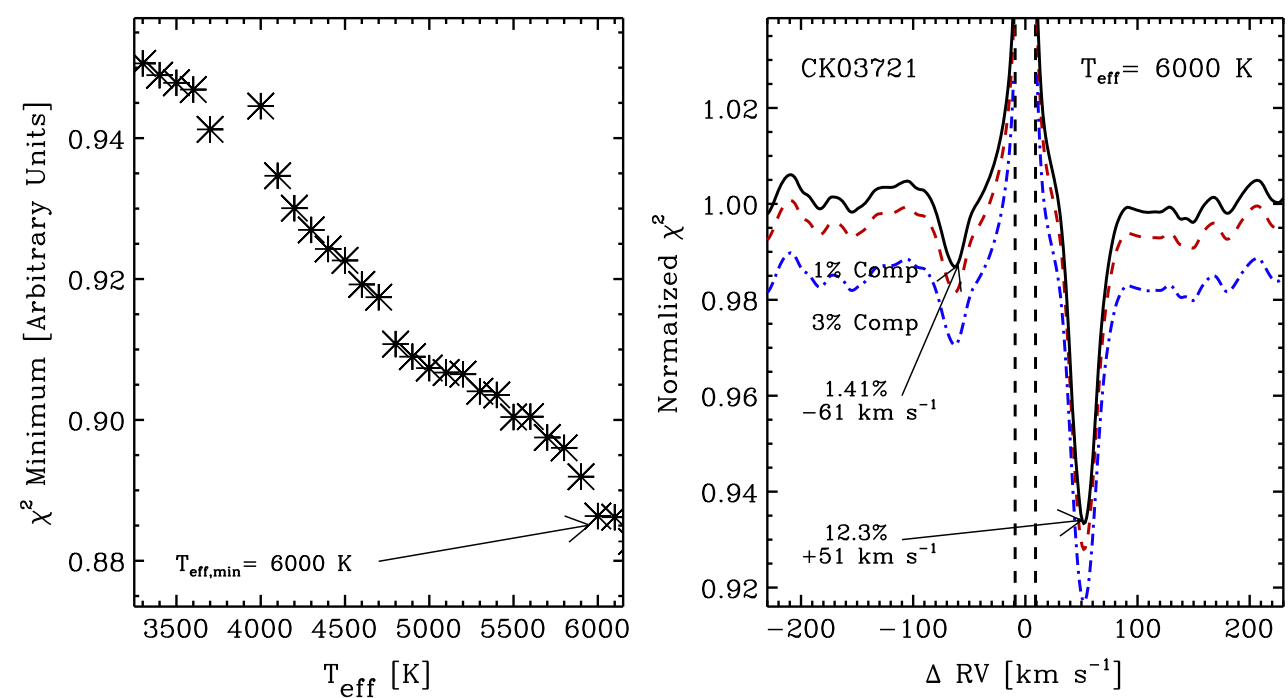

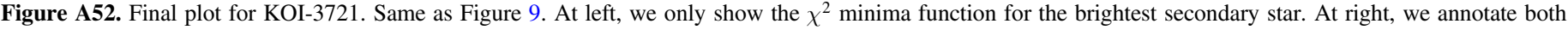
additional stars to KOI-3721 with an arrow. 

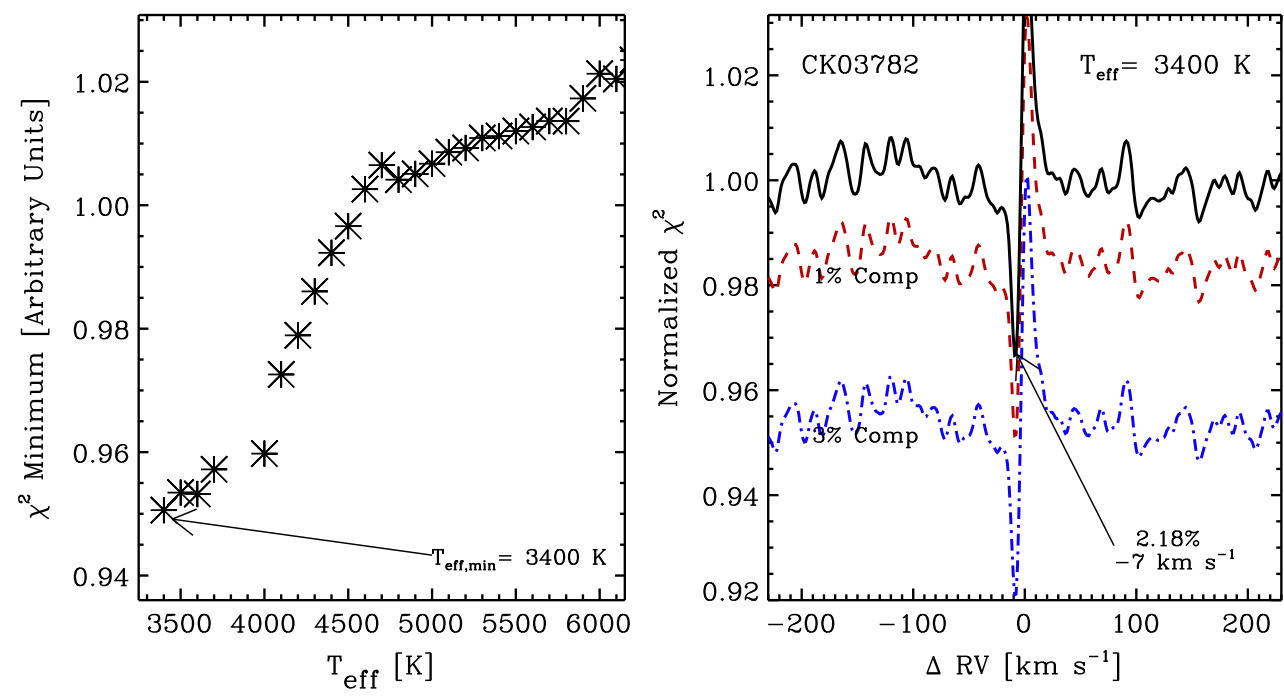

Figure A53. Final secondary star plot for KOI-3782. Same as Figure 9.
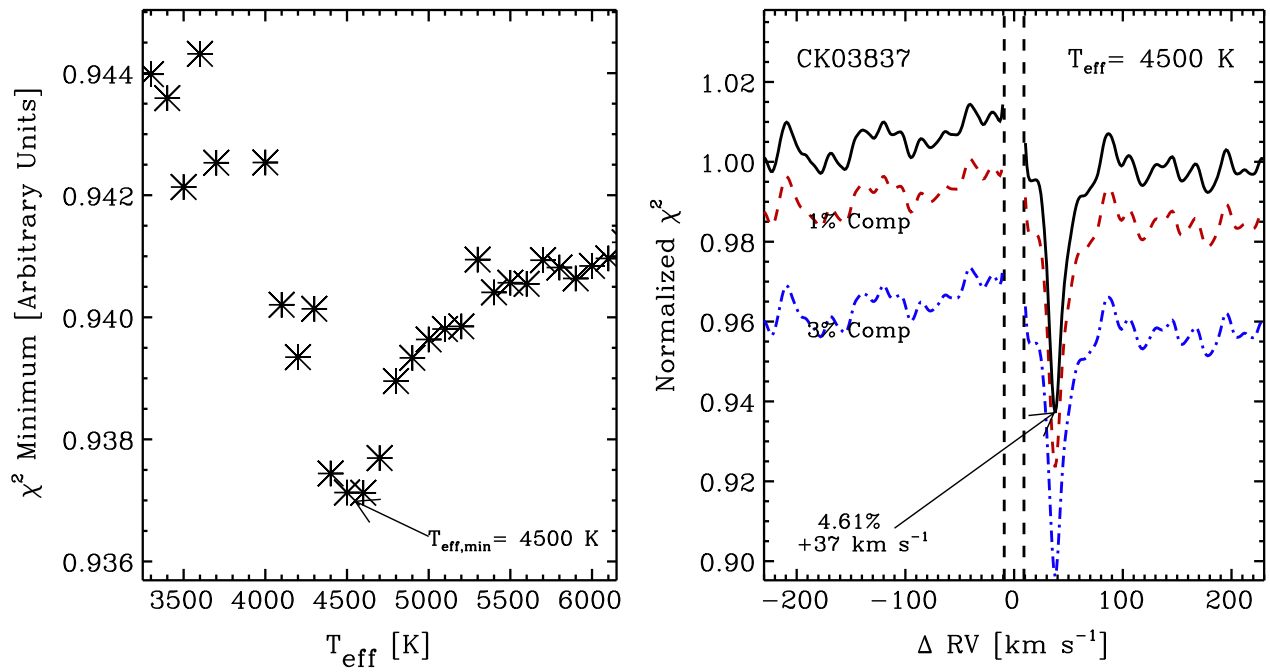

Figure A54. Final secondary star plot for KOI-3837. Same as Figure 9.
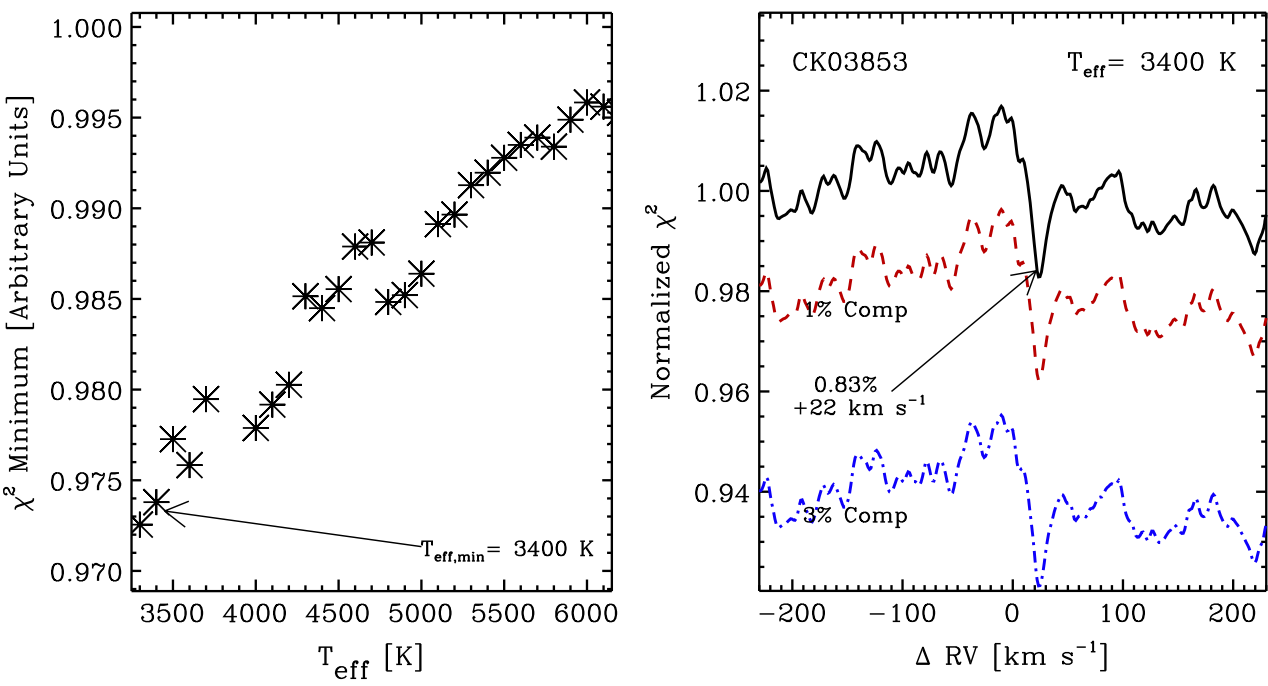

Figure A55. Final secondary star plot for KOI-3853. Same as Figure 9. 

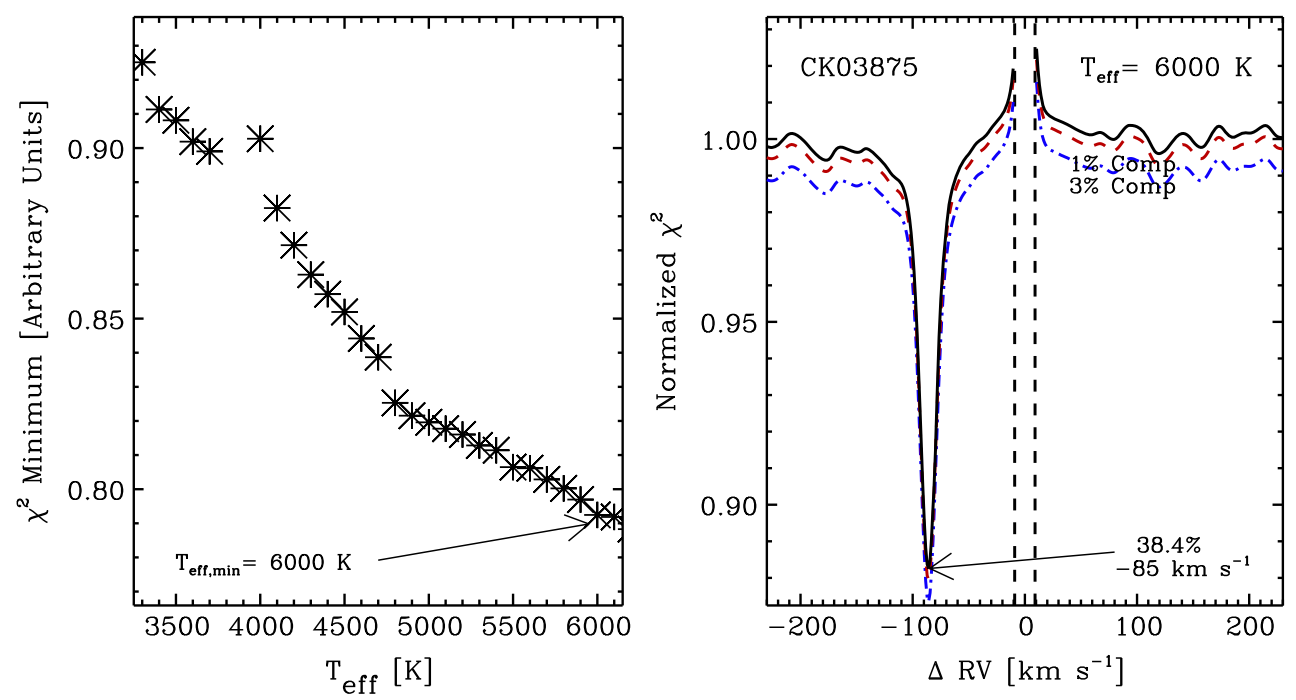

Figure A56. Final secondary star plot for KOI-3875. Same as Figure 9.
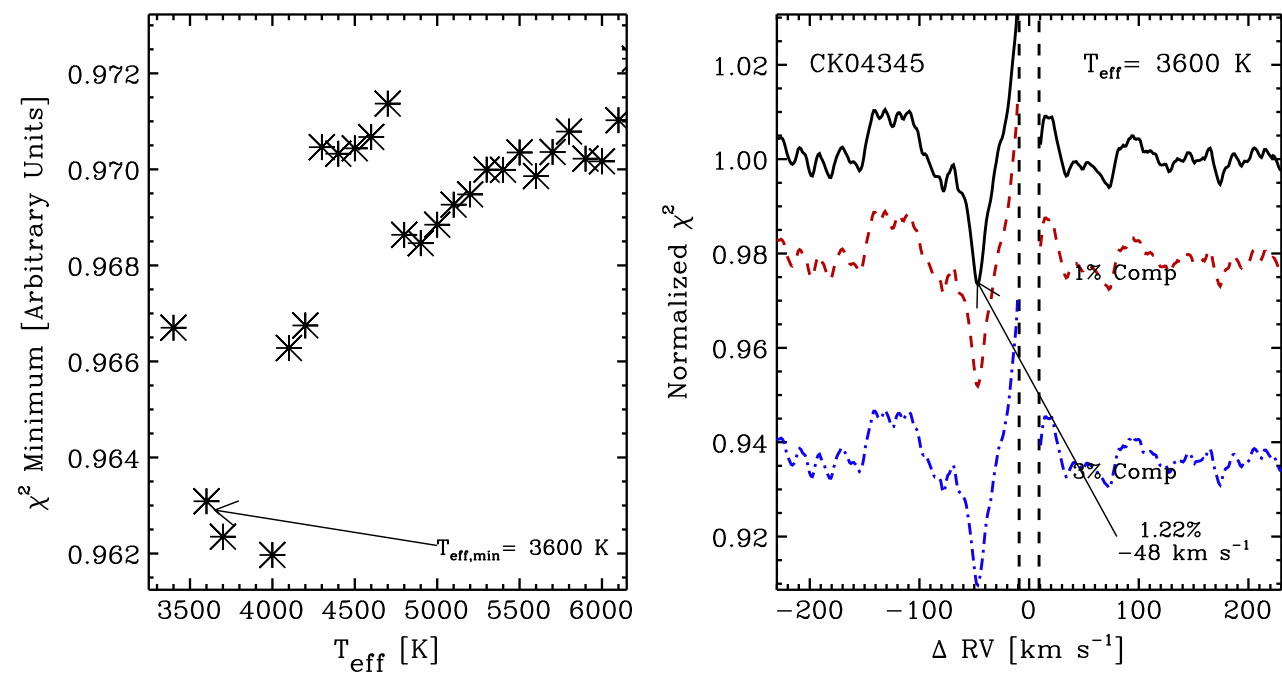

Figure A57. Final secondary star plot for KOI-4345. Same as Figure 9.
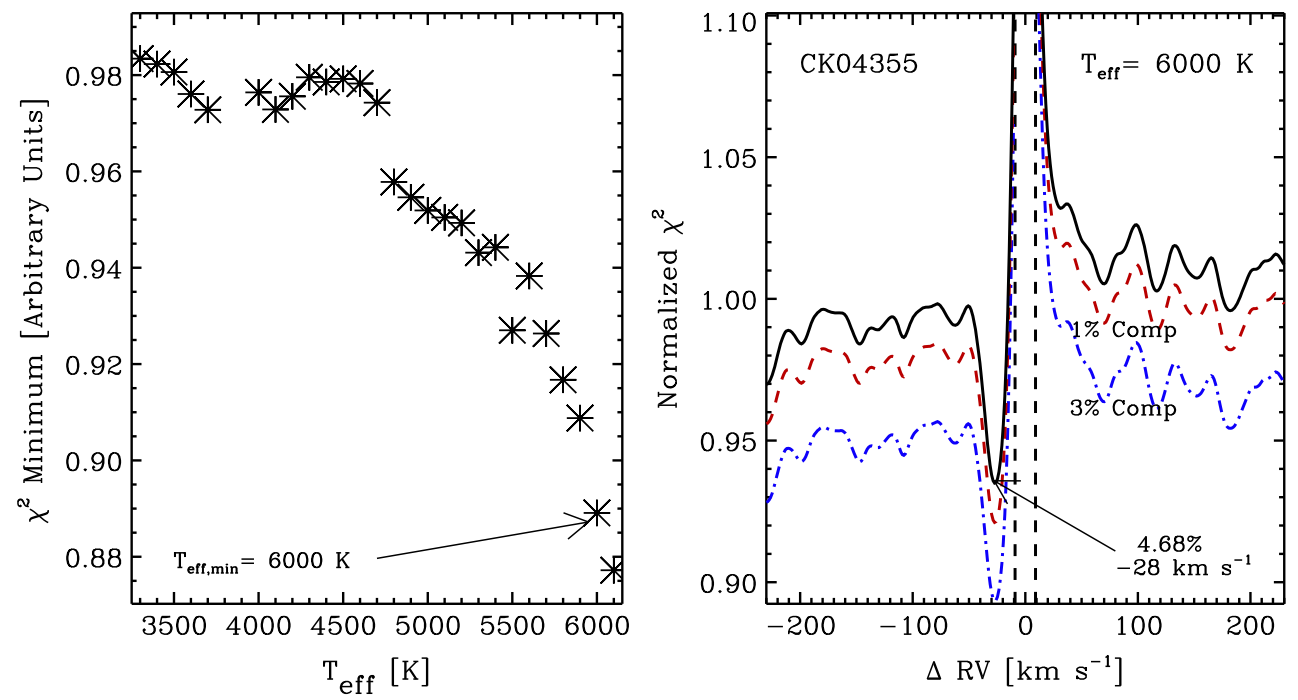

Figure A58. Final secondary star plot for KOI-4355. Same as Figure 9. 

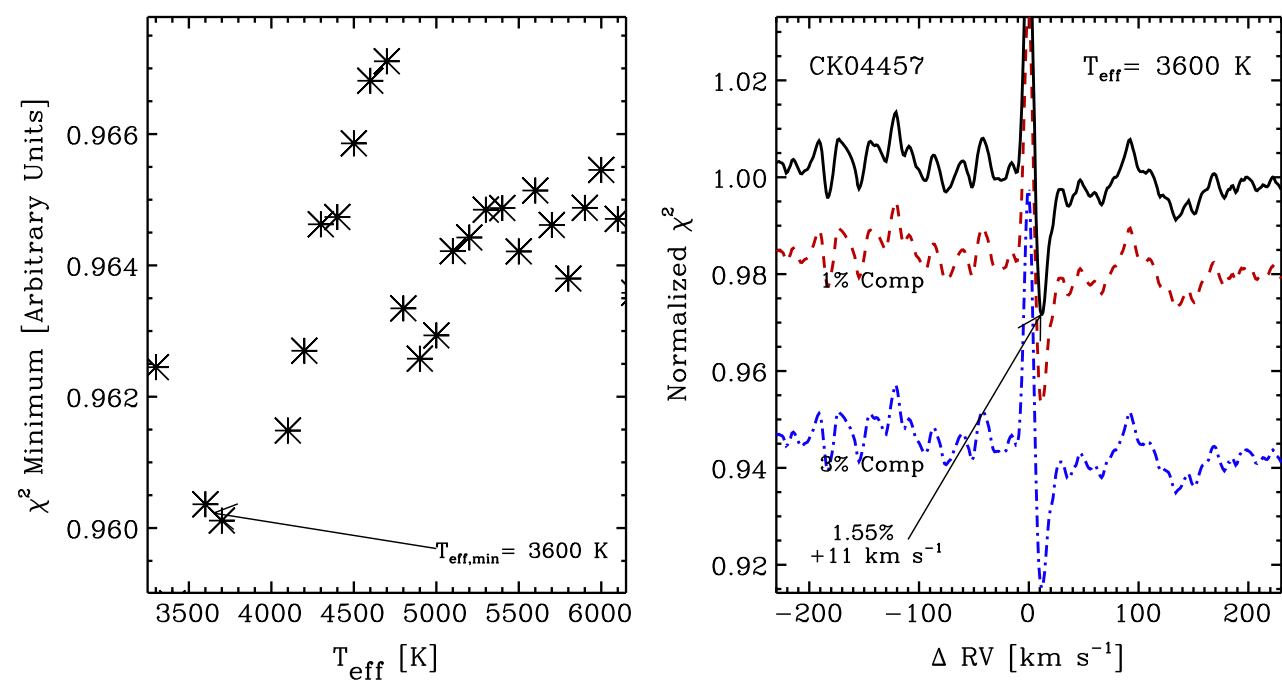

Figure A59. Final secondary star plot for KOI-4457. Same as Figure 9.
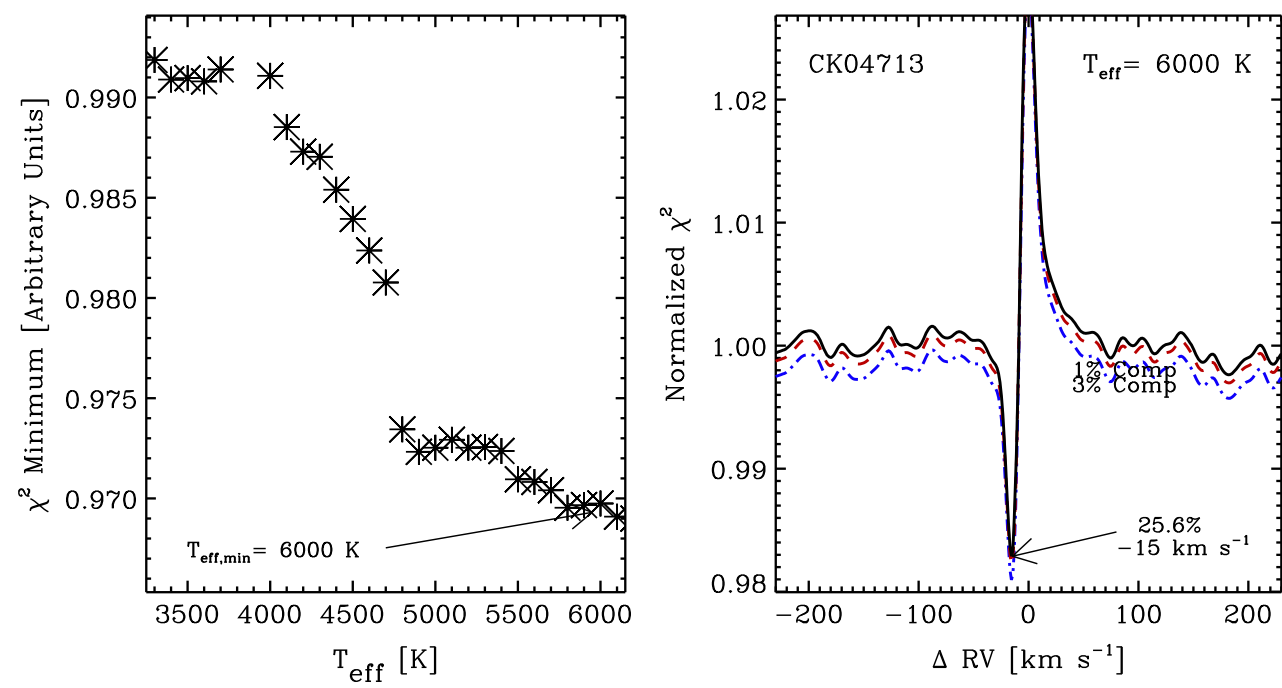

Figure A60. Final secondary star plot for KOI-4713. Same as Figure 9.
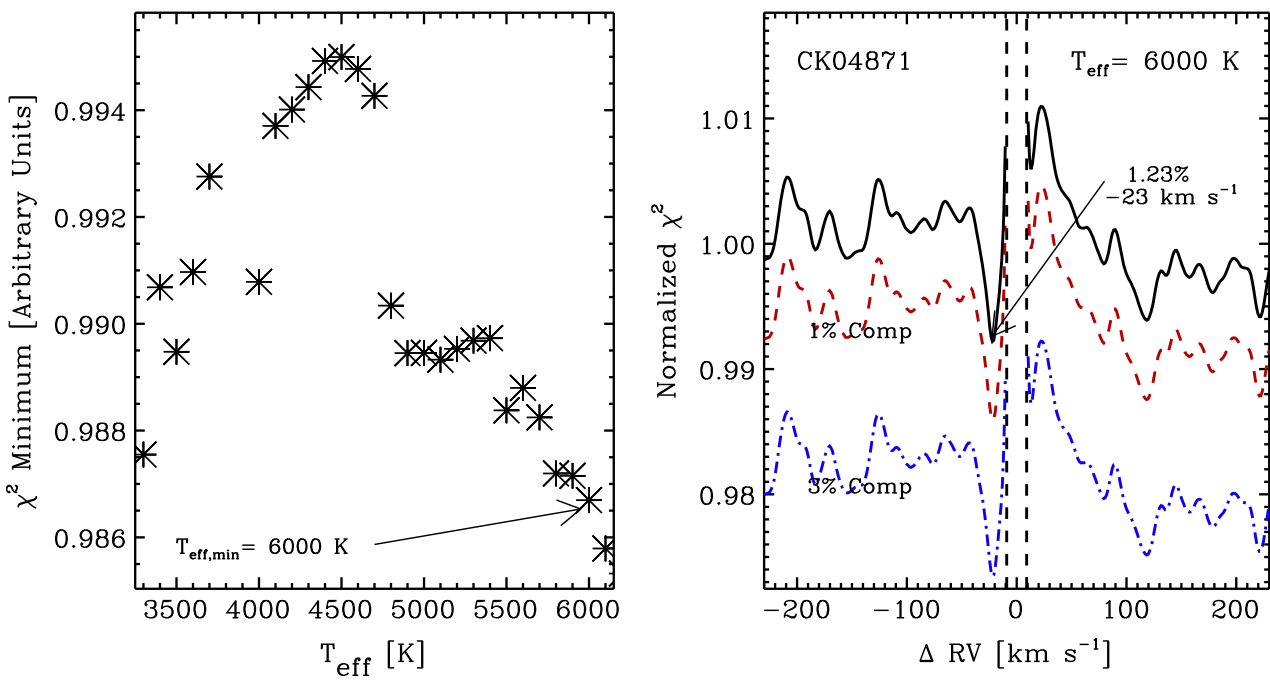

Figure A61. Final secondary star plot for KOI-4871. Same as Figure 9. 


\section{REFERENCES}

Ballard, S., Charbonneau, D., Fressin, F., et al. 2013, ApJ, 773, 98 Batalha, N. M., Rowe, J. F., Bryson, S. T., et al. 2013, ApJS, 204, 24 Díaz, R. F., Santerne, A., Sahlmann, J., et al. 2012, A\&A, 538, A113 Fressin, F., Torres, G., Charbonneau, D., et al. 2013, ApJ, 766, 81

Hirano, T., Sanchis-Ojeda, R., Takeda, Y., et al. 2012, ApJ, 756, 66

Law, N. M., Morton, T., Baranec, C., et al. 2014, ApJ, 791, 35

Marcy, G. W., \& Butler, R. P. 1992, PASP, 104, 270

Mazeh, T., Zucker, S., \& Smith, H. 1993, in Bulletin of the American Astronomical Society, 25, 1425

Morton, T. D., \& Johnson, J. A. 2011, ApJ, 738, 170

Raghavan, D., McAlister, H. A., Henry, T. J., et al. 2010, ApJS, 190, 1
Rappaport, S., Deck, K., Levine, A., et al. 2013, ApJ, 768, 33

Rojas-Ayala, B., Covey, K. R., Muirhead, P. S., \& Lloyd, J. P. 2012, ApJ, 748, 93

Santerne, A., Daz, R. F., Moutou, C., et al. 2012, A\&A, 545, A76

Slawson, R. W., Prša, A., Welsh, W. F., et al. 2011, AJ, 142, 160

Strassmeier, K. G., Weber, M., Granzer, T., \& Järvinen, S. 2012, AN, 333,663

Valenti, J. A., \& Fischer, D. A. 2005, ApJS, 159, 141

Wallace, L., Hinkle, K. H., Livingston, W. C., \& Davis, S. P. 2011, ApJS, 195,6

Wang, J., Xie, J.-W., Barclay, T., \& Fischer, D. A. 2014, ApJ, 783, 4

Welsh, W. F., Orosz, J. A., Aerts, C., et al. 2011, ApJS, 197, 4

Welsh, W. F., Orosz, J. A., Carter, J. A., et al. 2012, Natur, 481, 475 
ERRATUM: "DETECTION OF STARS WITHIN 0.8" OF KEPLER OBJECTS OF INTEREST" $(2015$, AJ, 149, 18)

Rea Kolbl ${ }^{1}$, Geoffrey W. Marcy ${ }^{1}$, Howard IsaACSOn ${ }^{1}$, and Andrew W. Howard ${ }^{2}$

2 University of California, Berkeley, CA 94720

Received 2014 December 29; accepted 2014 December 31; published 2015 February 2

Due to author's error during the publication process, fractional stellar separations are incorrectly referred to in inches. All instances of inches in the paper should have been quoted in arcseconds instead. This mistake occured in the title as well as throughout the main body of the paper. 